\title{
MULTIPLICITY OF THE ADJOINT REPRESENTATION IN SIMPLE QUOTIENTS OF THE ENVELOPING ALGEBRA OF A SIMPLE LIE ALGEBRA
}

\author{
ANTHONY JOSEPH
}

\begin{abstract}
Let $\mathfrak{g}$ be a complex simple Lie algebra, $\mathfrak{h}$ a Cartan subalgebra and $U(\mathfrak{g})$ the enveloping algebra of $\mathfrak{g}$. We calculate for each maximal two-sided ideal $J_{\max }(\lambda): \lambda \in \mathfrak{h}^{*}$ of $U(\mathfrak{g})$ the number of times the adjoint representation occurs in $U(\mathfrak{g}) / J_{\max }(\lambda)$. This is achieved by reduction via the Kazhdan-Lusztig polynomials to the case when $\lambda$ lies on a corner, i.e. is a multiple of a fundamental weight. Remarkably in this case one can always present $U(\mathfrak{g}) / J_{\max }(\lambda)$ as a (generalized) principal series module and here we also calculate its Goldie rank as a ring which is a question of independent interest. For some of the more intransigent cases it was necessary to use recent very precise results of Lusztig on left cells. The results are used to show how a recent theorem of Gupta established for "nonspecial" $\lambda$ can fail if $\lambda$ is singular. Finally we give a quite efficient procedure for testing if an induced ideal is maximal.
\end{abstract}

\section{INTRODUCTION}

1.1. This work is a continuation of [23] whose notation we adopt. It is a natural outgrowth of attempting to extend the validity of Gupta's theorem [12] and its converse to nonregular weights.

1.2. Let $\mathfrak{g}$ be a complex simple Lie algebra with triangular decomposition $\mathfrak{g}=$ $\mathfrak{n} \oplus \mathfrak{h} \oplus \mathfrak{n}^{-}$. Take $\lambda \in \mathfrak{h}^{*}$ dominant with respect to this decomposition and let $J_{\min }(\lambda)$ denote the annihilator of the Verma module with highest weight $\lambda-\rho$. After Duflo $[10,8.4 .4] J_{\min }(\lambda)$ is a minimal primitive ideal and all such ideals are so obtained. After Dixmier $[10,8.5 .8] U(\mathfrak{g})$ admits a unique maximal ideal $J_{\max }(\lambda)$ containing $J_{\min }(\lambda)$ and all maximal (two-sided) ideals of $U(\mathfrak{g})$ are so obtained. View $U(\mathfrak{g}) / J_{\max }(\lambda)$ as a $\mathfrak{g}$ module for the diagonal action. Our aim is to compute the multiplicity of the adjoint representation in $U(\mathfrak{g}) / J_{\max }(\lambda)$. This is quite easy for $\lambda$ regular and indeed by $[23,3.5,4.2,4.3]$ it equals rank $\mathfrak{g}$ less the number of simple roots $\alpha$ satisfying $\left(\alpha^{\vee}, \lambda\right)=1$. In general this estimate is only an upper bound and its true value is suprisingly more complicated to calculate.

1.3. Take a simple root $\alpha \in B$ and let $\bar{\omega}_{\alpha}$ denote its associated fundamental weight. We say that $\lambda \in \mathfrak{h}^{*}$ (dominant) lies on the $\alpha$-corner if it is a strictly

Received by the editors October 6, 1986 and, in revised form, January 7, 1987.

1980 Mathematics Subject Classification (1985 Revision). Primary 17B35; Secondary 22E46. 
positive integer multiple of $\bar{\omega}_{\alpha}$ and simply on a corner if it lies on some $\alpha$ corner. Our strategy is to first reduce our problem to the case when $\lambda$ lies on a corner. This is done essentially by translation principles in $\S 2$.

When $\lambda$ lies on a corner, it turns out rather miraculously that $J_{\max }(\lambda)$ is the annihilator of an induced module. Even more remarkably the induction may be done from the Lie algebra of an invariant parabolic subgroup, see 3.11. For the first assertion it turns out to be enough to show that the associated variety $\mathscr{V}\left(J_{\max }(\lambda)\right)$ of $J_{\max }(\lambda)$ is the closure of a Richardson orbit, which we verify in the following way. Suppose in fact that $\lambda$ lies on the $\alpha$-corner and let $B^{\prime}=B-\{\alpha\}$. Consider the $G$ orbit $\mathscr{O}_{B^{\prime}}$ generated by the regular nilpotent orbit in the Levi factor defined by $B^{\prime}$. We show that $\overline{\mathscr{O}}_{B^{\prime}} \subset \mathscr{V}\left(J_{\max }(\lambda)\right)$ and that equality holds if $\mathscr{O}_{B^{\prime}}$ is Richardson. When equality fails we use a symmetry property of the Kazhdan-Lusztig polynomials and results of Lusztig [24], Macdonald and Beynon-Lusztig [3] to calculate the Gelfand-Kirillov dimension of $U(\mathfrak{g}) / J_{\max }(\lambda)$. Comparison with the Gelfand-Kirillov dimension of quotient algebras over induced ideals proves the assertion in general. Curiously the Richardson orbit is always an even orbit in this latter case. Now assume more precisely that $\lambda=\bar{\omega}_{\alpha}$. It turns out that we cannot always further arrange that $J_{\max }(\lambda)$ is induced from a one-dimensional representation. However when this does hold we may then show that the quotient identifies with a principal series module and calculate the multiplicity of its $\mathfrak{k}$-types through Frobenius reciprocity. In general further tricks are needed. For example it is possible to relate the appearances of the adjoint representation for $\mathfrak{g}$ to the appearance of the short root vector representation in the "Langlands dual" of $\mathfrak{g}$. Finally for some last intransigent cases we are reduced to using the very precise description of left cells given by Lusztig [25, II, $\S 10$ and 28].

1.4. Via the equivalence of categories theorem for modules in the $\mathfrak{O}$ and Harish-Chandra categories [16, 6.26-27] we could in principle obtain the required multiplicities from the Kazhdan-Lusztig polynomials and the truth of the Kazhdan-Lusztig conjecture [16, 16.3]. However this turns out to be quite impractical except for checking one special case in type $C_{3}$. Of course we liberally use the Kazhdan-Lusztig polynomials to control the analysis (as already indicated above). Again the results on left cells mentioned above are also proved using the truth of their conjectures.

1.5. Take $\lambda$ on a corner. Though this is not essential for the computation of multiplicities, we show that $U(\mathfrak{g}) / J_{\max }(\lambda)$ is a principal series module and calculate its Goldie rank as a ring.

1.6. Our analysis leads to a third (4.6) and a fourth (4.9) way in which Gupta's theorem can fail for $\lambda$ nonregular. An example of the former is for $J_{\max }(\lambda): \lambda=$ $\bar{\omega}_{5}$ in type $D_{7}$ and of the latter for a certain nonmaximal ideal in $E_{8}$.

1.7. In 5.5 we found a remarkably efficient procedure (based on a result of Jantzen) for testing if an induced ideal is maximal. 
1.8. After this paper was completed Jantzen informed me in a letter dated August 12,1986 that in a few cases the multiplicities given in the table can be found in J. C. Jantzen, Zur Reduktion modulo $p$ unipotenter Charaktere endlicher Chevalley-Gruppen, Math. Z. 181 (1982), 97-128 as Verma module multiplicities. Thus in type $A_{n}$ these multiplicities can be read off from the formula on the top of p. 117 (op . cit.) which moreover contains some interesting additional information. In types $B_{n}$ and $C_{n}$ when the corner root is $\alpha_{n}$ the result appears in the middle of p. 120 (op.cit.). Although he did not calculate the multiplicities for the remaining corner roots $\alpha_{r}: r<n$, Jantzen informs me that his method gives in types $B_{n}$ and $C_{n}$ these multiplicities except in two families of cases:-

$$
\begin{aligned}
& \text { type } B_{n}, \quad r=2 m+1, \quad 3 m+1<n, \\
& \text { type } C_{n}, \quad r=2 m, \quad n<3 m,
\end{aligned}
$$

where our result is obtained as a lower bound.

1.9. The referee suggested that it would be helpful to give some insight into why the results are interesting. Here I can only give some general philosophy. One often thinks that problems for $\lambda$ singular (i.e., on a wall) should be simpler than for $\lambda$ regular or at least derivable from the regular case. However, we see here that this is not always so. The point is that to solve the problem for $\lambda$ singular by such a reduction we may have to solve a much more difficult and entirely intractable problem for $\lambda$ regular. This is true here and it also applies in studying unitary representations of complex groups which is much easier for $\lambda$ regular. Perhaps when we have a deeper understanding of for example the Kazhdan-Lusztig polynomials, this will no longer be so.

\section{TRANSLATION TO CORNERS}

Fix $-\lambda \in \mathfrak{h}^{*}$ dominant.

2.1. Recall $[23,4.3]$ that $B_{\lambda}^{1}:=\left\{\alpha \in B_{\lambda} \mid\left(\alpha^{\vee}, \lambda\right)=-1\right\}$. Given $\alpha \in B_{\lambda}$ let $\bar{\alpha}$ denote its affiliated root $[23,4.1]$. In $[23,4.1]$ we showed that the adjoint representation occurs in the simple Harish-Chandra module $V(-\lambda,-w \lambda): w \in W_{\lambda}-W_{\lambda}^{0}$ if and only if $w=s_{\bar{\alpha}}$ for some $\alpha \in B_{\lambda}^{1}$ and then it occurs with multiplicity one. Thus in order to calculate the multiplicity of the adjoint representation in $V(-\lambda,-\lambda) \cong U(\mathfrak{g}) / J_{\max }(\lambda)$ it is sufficient to calculate (see 5.1)

$$
\left[L(-\lambda,-\lambda): V\left(-\lambda,-s_{\bar{\alpha}} \lambda\right)\right]
$$

for each $\alpha \in B_{\lambda}^{1}$. Here $L(-\lambda,-\lambda)$ is a principal series module which by a classical result of Kostant may be identified with $U(\mathfrak{g}) / J_{\min }(\lambda)$. Of course $(*)$ is given by Kazhdan-Lusztig polynomials; but only in a very implicit fashion. Here we investigate how these multiplicities vary on passage to a corner. As pointed out in $[23,4.2]$ this is more delicate than it would seem at first sight. A slightly more general problem is analysed below. 
2.2. Let $P_{x, y}(q)$ denote the Kazhdan-Lusztig polynomial for the pair $x, y \in$ $W$. Take $B^{\prime}, B^{\prime \prime} \subset B$ and let $W_{B^{\prime}}$ (resp. $W_{B^{\prime \prime}}$ ) denote the subgroup of $W$ generated by the $s_{\alpha}: \alpha \in B^{\prime}$ (resp. $\left.B^{\prime \prime}\right)$.

Lemma. Suppose $B^{\prime} \cap B^{\prime \prime}=\varnothing$. Then $P_{x, y}(q)=P_{x w, y w}(q)$ for all $x, y \in W_{B^{\prime}}$, $w \in W_{B^{\prime \prime}}$.

Take $\alpha \in B^{\prime \prime}$ and set $s=s_{\alpha}$. It is enough to show that $P_{x w, y w}(q)=$ $P_{x w s, y w s}(q)$ whenever $l(w s)>l(w)$. Write $x^{\prime}=x w, y^{\prime}=y w$. By the Kazhdan-Lusztig algorithm $([16,16.2]$; but beware of Jantzen's definition of Bruhat order) we have

$$
P_{x^{\prime} s, y^{\prime} s}(q)=P_{x^{\prime}, y^{\prime}}(q)+q P_{x^{\prime} s, y^{\prime}}(q)-\sum_{z^{\prime} \in W \mid z^{\prime} s<z^{\prime}} \mu\left(z^{\prime}, y^{\prime}\right) q^{\left(l\left(y^{\prime}\right)-l\left(z^{\prime}\right)+1\right) / 2} P_{x^{\prime} s, z^{\prime}}(q) \text {. }
$$

Now $\mu\left(z^{\prime}, z^{\prime \prime}\right)=0$ unless $z^{\prime}<z^{\prime \prime}$, so we can assume $z^{\prime}<y^{\prime}$ in the sum. Again $P_{x^{\prime \prime}, y^{\prime \prime}}(q)=0$ unless $x^{\prime \prime} \leq y^{\prime \prime}$. In particular $P_{x^{\prime} s, y^{\prime}}=0$ and we can assume $x^{\prime} s<z^{\prime}$ in the sum.

Because $z^{\prime}<y^{\prime}$ we can write $z^{\prime}=z u: z \in W_{B^{\prime}}, u \in W_{B^{\prime \prime}}$. Since $B^{\prime} \cap B^{\prime \prime}=$ $\varnothing$ the inequalities $x w s \leq z u<y w$ imply that $x \leq z \leq y$ and $w s \leq u \leq w$. The latter is clearly impossible and so we conclude that all the terms in the sum vanish. This proves the lemma.

Remark. More generally $P_{x w, y v}(q)=P_{x, y}(q) P_{w, v}(q), \forall x, y \in W_{B^{\prime}}, w, v \in$ $W_{B^{\prime \prime}}$ given $B^{\prime} \cap B^{\prime \prime}=\varnothing$.

2.3. Assume for the moment that $\lambda$ is regular. Set $\mu=w_{\lambda} \lambda$ which is dominant. Take $B^{\prime}, B^{\prime \prime} \subset B_{\lambda}$. Assume $B^{\prime} \cap B^{\prime \prime}=\varnothing$.

Corollary. For all $x, y \in W_{B^{\prime}}$ one has

$$
[M(x \mu): L(y \mu)]=\left[M\left(x w_{B^{\prime \prime}} \mu\right): L\left(y w_{B^{\prime \prime}} \lambda\right)\right]
$$

where $w_{B^{\prime \prime}}$ denotes the unique longest element in $W_{B^{\prime \prime}}$.

Let $P_{x, y}(q)$ now refer to the Coxeter group $\left(W_{\lambda}, B_{\lambda}\right)$. Since $[M(x \mu): L(y \mu)]$ $=P_{x, y}(1)$, the assertion follows from 2.2 .

2.4. Set $\mu=w_{\lambda} \lambda$ as before; but drop the assumption that $\lambda$ is regular. Recall the definition of the circle product given in $[23,3.6]$.

Lemma. For all $w \in W_{\lambda}$ one has

$$
[L(-\lambda,-\lambda): V(-\lambda-w \lambda)]=\left[M(\mu): L\left(w_{\mu}^{0} \circ w_{\lambda} w^{-1} \lambda\right)\right],
$$

where $w_{\mu}^{0}$ denotes the unique longest element in $W_{\mu}^{0}:=\{w \in W \mid w \mu=\mu\}$.

Remark. One may also veiw $w_{\mu}^{0} \circ w_{\lambda} w^{-1}$ as the unique shortest element in the left $W_{\mu}^{0}$ coset of $W_{\lambda}$ containing $w_{\lambda} w^{-1}$. 
Since $\mu$ is dominant, $M(\mu)$ is projective in $\mathfrak{O}$ and we have an exact functor $M \mapsto L(M(\mu), M)$ from the $\mathfrak{O}$ category to the Harish-Chandra category. By [23, 5.2] we have isomorphisms $L(M(\mu), M(\mu)) \stackrel{\sim}{\longrightarrow} L(M(\lambda), M(\lambda)) \stackrel{\sim}{\longrightarrow}$ $L(-\lambda,-\lambda)$. By $[23,5.3]$ it follows that $L\left(M(\mu), L\left(w_{\mu}^{0} \circ w_{\lambda} w^{-1} \lambda\right)\right) \neq 0$ and then by $[24,4.7]$ we have isomorphisms

$$
\begin{aligned}
L\left(M(\mu), L\left(w_{\mu}^{0} \circ w_{\lambda} w^{-1} \lambda\right)\right) & \stackrel{\simeq}{\longrightarrow} V\left(-w_{\mu}^{0} \circ w_{\lambda} w^{-1} \lambda,-\mu\right) \\
& \cong V\left(-w_{\lambda} w^{-1} \lambda,-\mu\right) \cong V(-\lambda,-w \lambda)
\end{aligned}
$$

as required.

2.5. By [23, 3.5] we can assume $B_{\lambda}^{1} \subset B$ without loss of generality and that $B_{\lambda}^{0} \subset B$ because $-\lambda$ is dominant. Take $\alpha \in B_{\lambda}^{1}$ and let $B_{1}$ be the connected component of $B_{\lambda}^{0} \cup\{\alpha\}$ containing $\alpha$. By [23, 3.7] the affiliated root $\bar{\alpha}$ satisfies $s_{\bar{\alpha}} \subset W_{B_{1}}$. We wish to compare the expression in $2.1(*)$ with the corresponding expression in which $\mathfrak{g}$ is replaced by the Levi factor defined by $B_{1}$. We use a subscript $B_{1}$ on [:] to denote the latter. Of course here the choice of $B_{1}$ depends on $\lambda$. Set $B_{2}=B_{\lambda}^{0}-B_{3}, B_{3}=B_{1}-\{\alpha\}, B^{\prime}=-w_{\lambda} B_{1}, B^{\prime \prime}=-w_{\lambda} B_{2}$, $B^{\prime \prime \prime}=-w_{\lambda} B_{3}$. Then $w_{\mu}^{0}=w_{B^{\prime \prime}} w_{B^{\prime \prime \prime}}$ and $w_{B^{\prime \prime}}$ commutes with each $w \in W_{B^{\prime}}$.

Proposition. For all $w \in W_{B_{1}}$ one has

$$
[L(-\lambda,-\lambda): V(-\lambda,-w \lambda)]=[L(-\lambda,-\lambda): V(-\lambda,-w \lambda)]_{B_{1}} .
$$

Set $z=w_{\lambda} w^{-1} w_{\lambda} \in W_{B^{\prime}}$. Set $y=w_{B^{\prime \prime \prime} *} z \in W_{B^{\prime}}$. Observe that (notation $[23,3.6]\left(w_{\mu}^{0} \circ w_{\lambda} w^{-1}\right) \lambda=\left(w_{\mu^{*}}^{0} z\right) \mu=\left(w_{B^{\prime \prime \prime} *} z\right) \mu$. Then by 2.4 it is enough to show that

$$
[M(\mu): L(y \mu)]=[M(\mu): L(y \mu)]_{B^{\prime}} .
$$

After Jantzen $[15,2.14]$ we have

$$
\left[M\left(w_{\mu}^{0}(\mu+\nu)\right): L\left(y_{*} w_{\mu}^{0}(\mu+\nu)\right)\right]=\left[M\left(w_{\mu}^{0} \mu\right): L\left(y_{*} w_{\mu}^{0} \mu\right)\right]=[M(\mu): L(y \mu)]
$$

for all $y \in W_{\mu}, \nu \in P(R)^{+}$. Of course this result also holds for those quantities defined by the subscript $B^{\prime}$ except that $W_{\mu}$ must be replaced by $W_{B^{\prime}}$ and so $w_{\mu}^{0}$ must be replaced by $w_{B^{\prime \prime \prime}}$. Recall that $w_{\mu}^{0}=w_{B^{\prime \prime \prime}} w_{B^{\prime \prime}}$ and that $y_{*} w_{\mu}^{0}=$ $y_{*}\left(w_{B^{\prime \prime \prime}} w_{B^{\prime \prime}}\right)=\left(y_{*} w_{B^{\prime \prime \prime}}\right) w_{B^{\prime \prime}}$ because $y_{*} w_{B^{\prime \prime \prime}} \in W_{B^{\prime}}$ and $B^{\prime} \cap B^{\prime \prime}=\varnothing$. Take $\nu$ sufficiently large to make $\mu+\nu$ regular and then set $\xi=\mu+\nu$. From 2.3 we obtain

$$
\begin{aligned}
{\left[M\left(w_{\mu}^{0} \xi\right): L\left(y_{*} w_{\mu}^{0} \xi\right)\right] } & =\left[M\left(w_{B^{\prime \prime \prime}} \xi\right): L\left(y_{*} w_{B^{\prime \prime \prime}} \xi\right)\right] \\
& =\left[M\left(w_{B^{\prime \prime \prime}} \xi\right): L\left(y_{*} w_{B^{\prime \prime \prime}} \xi\right)\right]_{B^{\prime}}
\end{aligned}
$$

Here the last step follows from the fact that the Kazhdan-Lusztig polynomials $P_{x, y}(q): x, y \in W_{B^{\prime}}$ (which determine the $[M(x \xi): L(y \xi)]$ depend only on $W_{B^{\prime}}$ (and not on $W$ ). This follows from their recursive definition. Combining the above equalities proves the proposition. 
2.6. The result in 2.5 reduces the computation of the multiplicities defined by $2.1(*)$ to the case when $\lambda$ is integral and $B-B_{\lambda}^{0}=\{\alpha\}$, that is to the case when $\lambda$ lies on the $\alpha$-corner. This case will be analyzed in subsequent sections.

\section{SOME GENERAL THEOREMS}

3.1. It is clear that our final result must involve quite a lot of case by case analysis. Here we prove some general results which are needed for this analysis and which are also of independent interest.

3.2. Take $-\lambda \in \mathfrak{h}^{*}$ dominant. For each $w \in W_{\lambda}$ we set $\tau_{\lambda}(w)=\left\{\alpha \in B_{\lambda} \mid w \alpha \in\right.$ $\left.R^{-}\right\}$. Let $B^{\prime}$ be a subset of $\tau_{\lambda}\left(w^{-1}\right) \cap B$. (In our applications $\lambda$ will be integral, so $\left.\tau_{\lambda}(w) \subset B\right)$. Then if $(\alpha, w \lambda) \neq 0$ for all $\alpha \in B^{\prime}$ (for example if $\lambda$ is regular) we shall in fact have $\left(\alpha^{\vee}, w \lambda\right) \in \mathbf{N}^{+}$for all $\alpha \in B^{\prime}$ and so there exists a unique up to isomorphism finite dimensional simple $\mathfrak{p}_{B^{\prime}}$ module $V_{B^{\prime}}(w \lambda)$ with highest weight $w \lambda-\rho$. Set $M_{B^{\prime}}(w \lambda)=U(\mathfrak{g}) \otimes_{U\left(\mathfrak{p}_{B^{\prime}}\right)} V_{B^{\prime}}(w \lambda)$ and $I_{B^{\prime}}(w \lambda)=$ Ann $M_{B^{\prime}}(w \lambda)$ which we shall call an induced ideal. Following [19, 10.1 ] we call $J \supset J_{\min }(\lambda)$ almost induced if it is a minimal prime ideal over an induced ideal.

3.3. Let gr denote the gradation functor associated to the canonical filtration of $U(\mathfrak{g})$. Given an ideal $J$ of $U(\mathfrak{g})$ we let $\mathscr{V}(J)$ denote its associated variety which is by definition the zero variety in $\mathfrak{g}^{*}$ of gr $J$. Identify $\mathfrak{g}$ with $\mathfrak{g}^{*}$ through the Killing form. Given $B^{\prime} \subset B$ then $G \mathfrak{m}_{B^{\prime}} \subset \mathfrak{g}^{*}$ contains a unique dense nilpotent orbit and such an orbit is said to be of Richardson type. We need and prove the following result only for $-\lambda \in P(R)^{++}$. According to the referee it can fail for arbitrary antidominant $\lambda$, for example in type $B_{3}$ with $\lambda=\bar{\omega}_{1}+\frac{1}{2} \bar{\omega}_{2}+\bar{\omega}_{3}$.

Lemma. A primitive ideal $J \supset J_{\min }(\lambda)$ is almost induced if and only if $\mathscr{V}(J)$ is the closure of a Richardson orbit.

By $[4,4.6]$ one has $\mathscr{V}\left(I_{B^{\prime}}(w \lambda)\right)=G \mathfrak{m}_{B^{\prime}}$ and so by definition is the closure of a Richardson orbit. If $J$ is almost induced then $J$ is minimal over some $I_{B^{\prime}}(w \lambda)$. By $[16,17.13(6)]$ one has $\mathscr{V}(J)=\mathscr{V}\left(I_{B^{\prime}}(w \lambda)\right)$. This proves necessity.

For sufficiency suppose that $\mathscr{V}(J)=G \mathfrak{m}_{B^{\prime}}$ for some $B^{\prime} \subset B$.

Set $R^{\prime+}=\mathbf{N} B^{\prime} \cap R$ and

$$
p_{B^{\prime}}=\prod_{\alpha \in R^{\prime+}} \frac{\alpha}{(\alpha, \rho)} .
$$

Let $p$ denote the Goldie rank polynomial defined by the coherent family of ideals associated to $J$. (In particular $\operatorname{rk} U(\mathfrak{g}) / J=p(\lambda)$.) By [18, II, 5.4(i), 5.5 and $21,3.10]$ one has $p \in \mathbf{Q} W_{\lambda} p_{B^{\prime}}$. Set $D=\left\{w \in W_{\lambda} \mid w^{-1} B^{\prime} \subset R_{\lambda}^{+}\right\}$. It is a standard fact (see [7, p. 37, exercise 3]) that we can write each $w \in W_{\lambda}$ uniquely in the form $w=w_{2} w_{1}: w_{1} \in D, w_{2} \in W_{B^{\prime}}$. Since $w p_{B^{\prime}}=(-1)^{l(w)} p_{B^{\prime}}$ for each $w \in W_{B^{\prime}}$, it follows that $\mathbf{Q} W_{\lambda} p_{B^{\prime}}=\mathbf{Q}\left\{w^{-1} p_{B^{\prime}}: w \in D\right\}$. On the other 
hand for each $w \in D$, the induced ideal $I_{B^{\prime}}\left(w w_{\lambda} \lambda\right)$ is defined. We show that it is possible to choose $w \in D$ so that $J$ is minimal over $I_{B^{\prime}}\left(w w_{\lambda} \lambda\right)$.

Set $A_{y}=L\left(M_{B^{\prime}}(y \lambda), M_{B^{\prime}}(y \lambda)\right)$ where $y \in D w_{\lambda}$. This is a prime, noetherian ring [16, 15.21] satisfying $\operatorname{rk} A_{y}=\operatorname{dim} V_{B^{\prime}}(y \lambda)=\left(y^{-1} p_{B^{\prime}}\right)(\lambda)$. By the Goldie rank additivity principle $[16,12,3(4)]$ applied to embedding $U(\mathfrak{g}) / I_{B^{\prime}}(y \lambda) \hookrightarrow$ $A_{y}$ we conclude that the Goldie rank polynomials (which are linearly independent $\left[18\right.$, II, 5.5] associated to the minimal primitive ideals over $I_{B^{\prime}}(y \lambda)$ form a linear combination with certain positive integer coefficients equal to the polynomial defined by rk $A_{y}$ namely $y^{-1} p_{B^{\prime}}$. These observations prove the lemma.

3.4. We remark that an induced ideal need not be primitive and so it is not surprising that almost induced ideals are much more common than those induced ideals which are primitive. Again if an induced ideal $I_{B^{\prime}}(w \lambda)$ is primitive it does not mean that $M_{B^{\prime}}(w \lambda)$ needs to be simple or even that one can choose a simple induced module whose annihilator equals $I_{B^{\prime}}(w \lambda)$-for example, consider the induced ideals in type $A_{3}$ with Goldie rank polynomials $\alpha_{2}\left(\alpha_{1}+\alpha_{2}+\alpha_{3}\right)$ noting $[19,11.5]$ in this connection. Yet we have the

Lemma. Suppose $J_{\max }(\lambda)$ is almost induced, that is minimal over some induced ideal $I_{B^{\prime}}(w \lambda):=\operatorname{Ann} M_{B^{\prime}}(w \lambda)$. Then $J_{\max }(\lambda)=\sqrt{I_{B^{\prime}}(w \lambda)}$. Moreover one can choose $y \in W$ such that $M_{B^{\prime}}(y \lambda)$ is simple and $J_{\max }(\lambda)=I_{B^{\prime}}(y \lambda)$.

The first part is immediate. For the second part we remark that as $M_{B^{\prime}}(w \lambda)$ is defined for some $w \in W$ by hypothesis, there exists $y \in W$ minimal with respect to the Bruhat order < such that $M_{B^{\prime}}(y \lambda)$ is defined. Now $M_{B^{\prime}}(y \lambda)$ is simple because any proper simple submodule would be the image of some $M_{B^{\prime}}(x \lambda)$ with $x<y$. Finally $J_{\max }(\lambda) \supset I_{B^{\prime}}(y \lambda)$, whereas

$$
d\left(U(\mathfrak{g}) / J_{\max }(\lambda)\right)=d\left(U(g) / I_{B^{\prime}}(w \lambda)\right)=\operatorname{card} R-\operatorname{card} R^{\prime}=d\left(U(\mathfrak{g}) / I_{B^{\prime}}(y \lambda)\right)
$$

so we get equality by the primitivity of $I_{B^{\prime}}(y \lambda)$.

3.5. To check that $J_{\max }(\lambda)=\operatorname{Ann} M_{B^{\prime}}(y \lambda)$ for some $y \in W_{\lambda}$ it is enough by 3.3 and 3.4 to show that $\mathscr{V}\left(J_{\max }(\lambda)\right)=G \mathfrak{m}_{B^{\prime}}$. There may be several subsets $B^{\prime} \subset B$ satisfying the latter condition and each of these may give rise to an appropriate simple module. We shall eventually wish to choose $B^{\prime}$ and $y$ such that the embedding $U(\mathfrak{g}) / \operatorname{Ann} M_{B^{\prime}}(y \lambda) \hookrightarrow L\left(M_{B^{\prime}}(\lambda), M_{B^{\prime}}(\lambda)\right)$ is an isomorphism. This is rather more delicate.

3.6. In general $J_{\max }(\lambda)$ is not an induced ideal even when $\lambda$ is integral (unless it is also regular). For example in type $D_{4}$ one may choose $\lambda$ integral and on a wall such that $\mathscr{V}(J \max (\lambda))$ is the closure of the unique nilpotent orbit of minimal nonzero dimension and this is known not to be of Richardson type [17, Proposition 3.5 and $\S 6$, table]. Nevertheless we shall show that $J_{\max }(\lambda)$ is induced when $\lambda$ lies on a corner. This inevitably involves some case by case analysis. A first step is achieved by two technical results below. 
3.7. Take $w \in W$ and let $\mathscr{V}(w)$ denote the subvariety of $\mathfrak{n}$ defined in [20, 7.5]. For our present purposes we need only that $\overline{\mathscr{V}(w)}$ is the closure of the set defined by the action of the Borel subgroup $B$ (with Lie algebra $\mathfrak{b}$ ) on $\mathfrak{n} \cap w(\mathfrak{n})$.

Now assume $\lambda \in \mathfrak{h}^{*}$ is dominant, and take $J \supset J_{\min }(\lambda)$ primitive. By translation principles $[16,17,13(4)]$ we may choose $\mu \in \lambda+P(R)$ dominant, regular and $w \in W_{\lambda}$ such that $J(w \mu), J$ have the same associated variety. When $J=J_{\max }(\lambda)$ we may choose $w=w_{\lambda}^{0}$. In particular if $\lambda$ lies on the $\alpha$ corner then $J_{\max }(\lambda)$ and $J\left(w_{B^{\prime}} \mu\right): B^{\prime}=B-\{\alpha\}$ have the same associated variety for any $\mu \in P(R)^{++}$.

Given $B^{\prime} \subset B$ set $\mathfrak{n}_{B^{\prime}}=\mathfrak{r}_{B^{\prime}} \cap \mathfrak{n}$.

Lemma. Suppose $\mu \in P(R)^{++}$and $w \in W$. Then $\mathscr{V}(J(w \mu)) \supset \overline{G \mathscr{V}\left(w w_{B}\right)}$. In particular $\mathscr{V}\left(J\left(w_{B^{\prime}} \mu\right)\right) \supset \overline{G \mathfrak{n}}_{B^{\prime}}$.

We have $\mathscr{V}(J(w \mu)) \supset \overline{G \mathscr{V}(L(w \mu))}$ trivially and $\mathscr{V}(L(w \mu)) \supset \mathscr{V}\left(w w_{B}\right)$ (by $[20,8.15]$ - noting that $\mu$ is antidominant there). This proves the first part. Finally $\mathfrak{n} \cap w_{B^{\prime}} w_{B} \mathfrak{n}=\mathfrak{n} \cap w_{B^{\prime}} \mathfrak{n}^{-}=\mathfrak{n}_{B^{\prime}}$.

Remark. Except in type $A_{n}$ the above inclusion can be strict.

3.8. Take $\mu \in \mathfrak{h}^{*}$ dominant. The set $X_{\hat{\mu}}:=\left\{J \in \operatorname{Prim} U(\mathfrak{g}) \mid J \supset J_{\min }(\mu)\right\}$ is the fiber over $\hat{\mu} \in \mathfrak{h}^{*} / W$ for the map Prim $U(\mathfrak{g}) \rightarrow \operatorname{Max} Z(\mathfrak{g}) \stackrel{\sim}{\longrightarrow} \mathfrak{h}^{*} / W$ defined by $J \mapsto J \cap Z(\mathfrak{g})$. If $\mu \in P(R)^{+}$(resp. $\mu \in P(R)^{++}$) it is called an integral (resp. regular integral) fiber over Prim $U(\mathfrak{g})$. Recall [21, 3.10] for each $J \in \operatorname{Prim} U(\mathfrak{g})$ that $\mathscr{V}(J)$ is the closure of a nilpotent orbit and if $J \in X_{\hat{\mu}}$ we say that this nilpotent orbit belongs to the fiber $X_{\hat{\mu}}$. After Barbasch and Vogan $[1,2]$ one knows exactly which orbits belong to the regular integral fibers, indeed they are the special nilpotent orbits in the sense of Lusztig. For our purposes we shall only need to know that each Richardson orbit belongs to some regular integral fiber, a fact which is an easy consequence of $[14,4.6]$. The following is a direct proof of independent interest of a special case of Spaltenstein's duality on nilpotent orbits being implemented by the order-reversing duality (cf. [ 18 , III, 4.7]) on primitive ideals. This was checked essentially case by case (cf. [1, $2,4])$.

Proposition. Take $\mu \in P(R)^{++}$and $B^{\prime} \subset B$. Suppose $\overline{G \mathfrak{n}}_{B^{\prime}}$ is the closure of a special orbit, then $\mathscr{V}\left(J\left(w_{B^{\prime}} \mu\right)\right)=\overline{G \mathfrak{n}}_{B^{\prime}}$.

Since $\mathscr{V}\left(J\left(w_{B^{\prime}} \mu\right)\right)$ is an irreducible variety it is enough by 3.7 to prove equality of dimensions. However as this fails without the hypothesis on $\overline{G \mathfrak{n}}_{B}$, one can expect that a somewhat more roundabout argument is necessary. In fact we shall interpret the Borho-Jantzen-Duflo $\tau$-invariant for primitive ideals at the level of varieties.

Recall that $\mu$ is integral by hypothesis and set $\mathscr{L}=\{L(w \mu) \mid w \in W$ and $\left.\mathscr{V}(J(w \mu))=\overline{G \mathfrak{n}}_{B^{\prime}}\right\}$. The hypothesis on $\overline{G \mathfrak{n}}_{B^{\prime}}$ means exactly that $\mathscr{L}$ is nonempty. For each $L \in \mathscr{L}$ let $p_{v(L)}$ denote the polynomial in $S(\mathfrak{h})$ assigned 
to $\mathscr{V}(L)$ by the procedure of $[20, \S 2]$. By $[20, \S 5]$ the space $\mathbf{Q}\left\{p_{v(L)}: L \in \mathscr{L}\right\}$ is a $W$ submodule of $S(\mathfrak{h})$ (in fact it is even a Goldie rank representation).

Take $L \in \mathscr{L}$. Because $L$ is a highest weight module we have $\mathscr{V}(L) \subset$ $\mathfrak{n} \cap \overline{G \mathfrak{n}}_{B^{\prime}}$. Moreover by the Spaltenstein-Steinberg equality $[16,17.14(6)]$ and $[16,10.9,17.10]$ we have $\operatorname{dim} \overline{G \mathfrak{n}}_{B^{\prime}}=2 \operatorname{dim}\left(\mathfrak{n} \cap \overline{G \mathfrak{n}}_{B^{\prime}}\right) \geq 2 \operatorname{dim} \mathscr{V}(L)=2 d(L)=$ $d(U(\mathfrak{g}) / \operatorname{Ann} L)=\operatorname{dim} \overline{G \mathfrak{n}}_{B^{\prime}}$ and so we have equality of dimension. Let $\mathscr{V}_{i}: i \in$ $\mathcal{F}$ denote the irreducible components of $\mathfrak{n} \cap \overline{G \mathfrak{n}}_{B^{\prime}}$ (which after Spaltenstein [31] is equidimensional) and let $p_{v_{i}}$ be the polynomial associated to $\mathscr{V}_{i}$ as in [20, §2]. Now $\mathscr{V}(L)$ need not be irreducible (Tanisaki [32]) and this causes some technical difficulties; yet it does follow from the above that $\mathscr{V}(L)$ admits some of the $\mathscr{V}_{i}$ as irreducible components. Moreover by the remark in the last sentence of $[20,3.2]$ every $\mathscr{V}_{i}: i \in \mathscr{I}$ does occur in some $\mathscr{V}(L): L \in \mathscr{L}$ as an irreducible component. By [20,§3] the space $\mathbf{Q}\left\{p_{v_{i}}: i \in \mathscr{I}\right\}$ is a $W$ module which by the above contains $\mathbf{Q}\left\{p_{v_{L}}: L \in \mathscr{L}\right\}$ as a submodule. (In fact by a result of Hotta [13] the former is a simple module (the Springer representation) and so we have equality.)

It follows from $[20,7.6]$ that $\overline{B \mathfrak{n}}_{B^{\prime}}$ is the closure of some $\mathscr{V}_{i}(i \in \mathscr{I})$. We let $p$ denote the corresponding polynomial $p_{v_{i}}$. Take $\alpha \in B$. We show that

$$
p \text { has a zero on the } \alpha \text {-wall } \Leftrightarrow \alpha \in B-B^{\prime} \text {. }
$$

Suppose $p$ has a zero on the $\alpha$-wall. We show that $s_{\alpha} p=-p$. If not, then by $[20,3.1]$ we have

$$
s_{\alpha} p=\sum_{i \in \mathcal{I}} n_{i} p_{v_{i}}
$$

where the $n_{i}$ are nonnegative integers.

The hypothesis on $p$ exactly means that $\alpha$ divides $p$. Let us show that $\alpha^{2}$ does not divide $p$. By Hotta's result [19] $p$ is a $W$ harmonic polynomial and the above assertion is a general property of such polynomials. Indeed the space of $W$ harmonic polynomials $\mathscr{H}$ is stable under differentiation so if $q \in \mathscr{H}$ is divisible by $\alpha^{2}$ it follows (by differentiation with respect to the remaining simple roots) that $\alpha^{k} \in \mathscr{H}$ for some $k \geq 2$ and hence that $\alpha^{2} \in \mathscr{H}$. Yet the invariant differential operator $\sum_{\beta \in W_{\alpha}} \frac{\partial^{2}}{\partial \beta^{2}}$ applied to $\alpha^{2}$ gives up to a nonzero scalar $\sum_{w \in W}(w \alpha, \alpha)^{2}$. This is nonvanishing and so we conclude that $\alpha^{2} \notin \mathscr{H}$. This proves our assertion on $W$ harmonic polynomials.

We call $\xi \in P(R)^{++}$sufficiently large if each $\left(\beta^{\vee}, \xi\right): \beta \in B$ is sufficiently large. From the definition of the $p_{v_{i}}$ we have that $p_{v_{i}}(\xi)>0$ for all $i \in$ $\mathscr{I}$ and all $\xi \in P(R)^{++}$sufficiently large. Again the $p_{v_{i}}$ are homogeneous polynomials $[20,2.3$ (iii)]. Consider the homogeneous polynomial $p / \alpha$. Set $P_{\alpha}=\left\{\eta \in P(R)^{+} \mid(\eta, \alpha)=0\right.$ and $\left(\eta, \beta^{\vee}\right)$ is sufficiently large for all $\beta \in B-$ $\{\alpha\}\}$. We have shown that $p / \alpha$ does not vanish identically on $P_{\alpha}$ (otherwise it would be divisible by $\alpha$ ) and so we can assume without loss of generality that $(p / \alpha)(\eta) \neq 0, \forall \eta \in P_{\alpha}$. Yet $p / \alpha$ is homogeneous and satisfies $(p / \alpha)(\xi)>0$ 
for all $\xi \in P(R)^{++}$sufficiently large so we easily conclude that $(p / \alpha)(\eta)>0$ for all $\eta \in P_{\alpha}$. Now for $\eta \in P_{\alpha}$ we have $s_{\alpha} \eta=\eta$ and so $\left(\left(s_{\alpha} p\right) / \alpha\right)(\eta)=$ $-s_{\alpha}(p / \alpha)(\eta)=-(p / \alpha)(\eta)<0$. We conclude (as above) that $\left(s_{\alpha} p\right)(\xi)<0$ for all $\xi \in P(R)^{++}$sufficiently large. This contradicts $(* *)$. We conclude that $s_{\alpha} p=-p$. Conversely if $s_{\alpha} p=-p$ then (as is well known) $\alpha$ divides $p$ and so $p$ has a zero on the $\alpha$-wall.

Finally let $\mathfrak{m}_{\alpha}$ denote the nilradical of $\mathfrak{p}_{\alpha}$. Since $\mathfrak{m}_{\alpha}$ is $B$ stable we conclude that $\overline{B \mathfrak{n}}_{B^{\prime}} \subset \mathfrak{m}_{\alpha}$ if and only if $\alpha \in B-B^{\prime}$. Thus $(*)$ results from the previous equivalence and the two case decomposition in the proof of $[20,3.1]$.

Choose $L \in \mathscr{L}$ such that $\overline{B \mathfrak{n}}_{B^{\prime}}$ occurs as an irreducible component of $\mathscr{V}(L)$. We can write $L=L(w \mu)$ for some $w \in W$. Take $\alpha \in B^{\prime}$. We can write $p_{v(L)}=\sum_{i \in \mathcal{I}} m_{i} p_{v_{i}}$ where the $m_{i}$ are nonnegative integers and $m_{i} \neq 0$ if and only if $\mathscr{V}_{i}$ is a component of $\mathscr{V}(L)$. Now as above we can show that $p_{v_{i}}(\eta) \geq 0$ for all $\eta \in P_{\alpha}$ with equality if and only if $p_{v_{i}}$ has a zero on the $\alpha$-wall. Hence our hypothesis and $(*)$ imply that $p_{v(L)}$ does not have a zero on the $\alpha$-wall. From [20, 5.2] we conclude that the Goldie rank polynomial $p_{w^{-1}}$ does not have a zero on the $\alpha$-wall. The latter is exactly the condition that the ideal $J\left(w^{-1} \mu\right)$ does not degenerate to $U(\mathfrak{g})$ under Borho-Jantzen translation to the $\alpha$-wall. Yet by Borho-Jantzen [5, 2.17c] $J\left(w_{B^{\prime}}, \mu\right)$ is the unique maximal ideal of $U(\mathfrak{g})$ containing $J_{\min }(\mu)$ with the property that it does not degenerate to $U(\mathfrak{g})$ under Borho-Jantzen translation to each of the $\alpha$-walls $\alpha \in B^{\prime}$. We conclude that $J\left(w^{-1} \mu\right) \subset J\left(w_{B^{\prime}} \mu\right)$. Thus by choice of $w$ and the irreducibility $[21,3.8]$ of $\mathscr{V}\left(J\left(w^{-1} \mu\right)\right)$ we have

$$
\overline{G \mathfrak{n}}_{B^{\prime}}=\mathscr{V}\left(J\left(w^{-1} \mu\right)\right) \supset \mathscr{V}\left(J\left(w_{B^{\prime}} \mu\right)\right) \supset \overline{G \mathfrak{n}}_{B^{\prime}}
$$

and this gives the assertion of the proposition.

3.9. Take $\alpha \in B$ and set $B^{\prime}=B-\{\alpha\}$.

Theorem. Take $\lambda \in P(R)^{+}$on the $\alpha$-corner. If $\overline{G \mathfrak{n}}_{B^{\prime}}$, coincides with some Richardson orbit closure $G \mathfrak{m}_{B^{\prime \prime}}: B^{\prime \prime} \subset B$, then $J_{\max }(\lambda)$ is the annihilator of a simple module induced from a finite dimensional simple $U\left(\mathfrak{p}_{B^{\prime \prime}}\right)$ module.

This follows from 3.3, 3.4 and 3.8.

3.10. Take $\lambda \in P(R)^{+}$on the $\alpha$-corner. The hypothesis of Theorem 3.9 is not always satisfied; yet its conclusion always holds. To treat the general case we first calculate the Gelfand-Kirillov dimension of $U(\mathfrak{g}) / J_{\max }(\lambda)$.

Let $\mathscr{H}$ denote the space of $W$ harmonic polynomials in $S(\mathfrak{h})$. Then $\mathscr{H}$ is a $W$ module graded by degree. For each $\tau \in \hat{W}$, let $l_{\tau}$ (resp. $h_{\tau}$ ) denote the lowest (resp. highest) degree for which $\tau$ occurs in $\mathscr{H}$. These numbers (and indeed more precise information) were calculated in type $A_{n}$ by Steinberg, for classical Lie algebras by Lusztig [25, §2], for $F_{4}$ by Macdonald and for the remaining exceptional cases by Beynon and Lusztig [3]. An appropriate pairing on $\mathscr{H}$ involving the product of the positive roots (which transforms like the 
sign representation $s n$ ) implies that

$$
l_{\tau \otimes s n}+h_{\tau}=\operatorname{card} R^{+} .
$$

Take $B^{\prime} \subset B$ and set $R^{\prime}=\mathbf{Z} B^{\prime} \cap R, R^{++}=R^{\prime} \cap R^{+}, p_{B^{\prime}}=\prod_{\alpha \in R^{\prime+}} \alpha$. After Macdonald [30], we have that $\mathbf{Q} W p_{B^{\prime}}$ is a simple $W$ module say of type $\tau\left(B^{\prime}\right)$ and furthermore his analysis shows that $l_{\tau\left(B^{\prime}\right)}=\operatorname{deg} p_{B^{\prime}}=\operatorname{card} R^{\prime+}$. Now more specifically take $B^{\prime}=B-\{\alpha\}$. We give in the table along the column labelled "degree" the value of $l_{\tau\left(B^{\prime}\right) \otimes s n}$ for each Lie algebra and each simple root. This was calculated from $(*)$ and $h_{\tau\left(B^{\prime}\right)}$ determined as follows. For the exceptional Lie algebras one is very nearly able to compute $h_{\tau\left(B^{\prime}\right)}$ from the above formula for $l_{\tau\left(B^{\prime}\right)}$ and the tables of Beynon-Lusztig [3]—essentially the value of $l_{\tau\left(B^{\prime}\right)}$ identifies $\tau\left(B^{\prime}\right)$. The remaining ambiguities (which arise only because Beynon and Lusztig lacked the prescience to give their results in a manner best suited to our purposes) can be resolved either by further use of [26, I] and the fact that after $[1,2]$ the representations concerned must be special, or by writing to Lusztig. For the classical Lie algebras we used [25, §2] to calculate $h_{\tau\left(B^{\prime}\right)}$. We describe this in more detail in 6.4-6.8. Let $d$ denote Gelfand-Kirillov dimension.

Lemma. Take $\mu \in P(R)^{++}$and $B^{\prime} \subset B$. Suppose $\lambda \in P(R)^{+}$satisfies $B^{\prime}=$ $\{\alpha \in B \mid(\lambda, \alpha)=0\}$. Then

$$
d\left(U(\mathfrak{g}) / J_{\max }(\lambda)\right)=d\left(U(\mathfrak{g}) / J\left(w_{B^{\prime}} \mu\right)\right) \geq \operatorname{card} R-2 l_{\tau\left(B^{\prime}\right) \otimes s n} .
$$

The equality is due to Borho-Jantzen [5, combine 2.11 and $2.17 \mathrm{c}$ ]. The inequality follows from the theory of Goldie rank polynomials and an inversion property of the Kazhdan-Lusztig polynomials. It occurs implicitly somewhere in $[1,2]$; but to spare the reader we indicate briefly its proof.

Let $p$ denote the Goldie rank polynomial associated to the coherent family of ideals $J\left(w_{B^{\prime}} \mu\right): \mu \in P(R)^{++}$(which determines the Goldie ranks of the corresponding quotient algebras). By [16, 10.9 and 18, II, 5.1] we have $d\left(U(\mathfrak{g}) / J\left(w_{B^{\prime}} \mu\right)\right)=2 d\left(L\left(w_{B^{\prime}} \mu\right)\right)=2\left(\operatorname{card} R^{+}-\operatorname{deg} p\right)$, so we only have to show that $\operatorname{deg} p \leq l_{\tau\left(B^{\prime}\right) \otimes s n}$. This follows from the fact that $\tau\left(B^{\prime}\right) \otimes s n$ occurs in the left cell containing $w_{B^{\prime}}$ [2, Proposition 2.2.5] and that the Goldie rank polynomial $p$ satisfies $\operatorname{deg} p=\min l_{\tau}$ where $\tau$ runs through the irreducible representations occurring in the corresponding left cell [18, II, $\S \S 2,5]$. One also has equality if and only if $\mathbf{Q} W p$ is of type $\tau\left(B^{\prime}\right) \otimes s n$. Though we do not need to know this, Barbasch and Vogan $[1,2]$ showed that the latter only fails in three cases (one in type $E_{7}$ and two in type $E_{8}$ ). We check independently (see below) that equality always holds if $\operatorname{card} B^{\prime}=\operatorname{card} B-1$.

3.11. Take $B^{\prime} \subset B$. After Richardson the action of $\mathfrak{p}_{B^{\prime}}$ on its nilradical $\mathfrak{m}_{B^{\prime}}$ admits a unique dense orbit, say $G X$. Let $P_{B^{\prime}}$ denote the parabolic subgroup of $G$ with Lie algebra $\mathfrak{p}_{B^{\prime}}$ and $G^{X}$ (resp. $P_{B^{\prime}}^{X}$ ) the centralizer of $X$ in $G$ 
(resp. in $P_{B^{\prime}}$ ). We shall say that $\mathfrak{p}_{B^{\prime}}$ is an invariant parabolic subalgebra if $P_{B^{\prime}}^{X}=G^{X}$.

Theorem. Take $\lambda \in P(R)^{+}$on a corner. Then $J_{\max }(\lambda)$ is the annihilator of a simple module induced from a finite dimensional simple module of an invariant parabolic subalgebra.

Fix $\alpha \in B$ and set $B^{\prime}=B-\{\alpha\}$. The proof divides into two cases. First if $\overline{G \mathfrak{n}}_{B}$, is a Richardson orbit closure. Here let us recall briefly how to calculate the Dynkin data of the unique dense orbit in $G \mathfrak{n}_{B^{\prime}}$. Set $X=\sum_{\alpha \in B^{\prime}} X_{\alpha}$. It is well known that $G X$ is the unique dense nilpotent orbit in the Levi factor $\mathfrak{r}_{B^{\prime}}$ of the parabolic subalgebra $\mathfrak{p}_{B^{\prime}}$. In particular $G X \cap \mathfrak{n}_{B^{\prime}}$ is dense in $\mathfrak{n}_{B^{\prime}}$ and so $\overline{G X}=\overline{G \mathfrak{n}}_{B^{\prime}}$. Furthermore applying the Jacobson-Morozov theorem to $X \in \mathfrak{r}_{B^{\prime}}$ we can find an $\mathfrak{s}$-triple $(X, H, Y)$ in $\mathfrak{r}_{B^{\prime}}$. We have $H=[X, Y] \in\left[\mathfrak{r}_{B^{\prime}}, \mathfrak{r}_{B^{\prime}}\right]$ and $\left[H, X_{\beta}\right]=2 X_{\beta}$ for all $\beta \in B^{\prime}$ (see for example [10,8.1.1]). These conditions determine $H \in \mathfrak{h}$ uniquely. Let $\rho_{B^{\prime}}$ and $\bar{\omega}_{B^{\prime}}: \beta \in B^{\prime}$ denote respectively the half sum of the positive roots and the fundamental weights calculated in $\mathfrak{r}_{B^{\prime}}$. One has $H=\sum\left\{H_{\alpha}: \alpha \in B^{\prime}\right\}$ but it is more convenient to normalize the short roots by $(\alpha, \alpha)=2$ calling $\beta$ long if $(\beta, \beta)>2$. Then (outside $G_{2}$ ) we have

$$
H=H_{\delta}, \quad \text { where } \delta=2 \rho_{B^{\prime}}-\sum_{\beta \in B^{\prime} \text { (long) }} \bar{\omega}_{\beta} .
$$

From the relation (valid outside $G_{\hat{z}}$ )

$$
\left(\beta, \alpha^{\vee}\right)= \begin{cases}2: & \alpha \text { short }, \beta \text { long } \\ 1: & \text { otherwise }\end{cases}
$$

we may calculate $\left[H, X_{\alpha}\right]$ which is a negative integer multiple of $X_{\alpha}$. In the table this is given for each Lie algebra $\mathfrak{g}$ and each simple root under the heading "coefficient". The corresponding orbit can be easily worked out given this data and the results appear under the heading "orbit(1)" in the table. Here for the classical Lie algebras we use the notation in Carter $[8,13.1]$ and for the exceptional Lie algebras the usual Dynkin data $[8,13.1]$.

If the coefficient is an even integer then the orbit is an even orbit in the sense of Dynkin and automatically of Richardson type. Moreover from the subset $R_{0}$ of roots which have zero weight relative to $H$ we can pick a basis $B_{0} \subset R_{0} \cap R^{+}$ of simple roots and this determines the so-called Dynkin parabolic subalgebra. The latter is defined to be the span of nonnegative weight spaces of $H$. It is well known to always be an invariant parabolic subalgebra. In this case the Dynkin parabolic is also a polarization to the orbit. This proves the assertion of the theorem in this case. Under the heading $B^{\prime \prime}$ we have further computed a Weyl group conjugate of $B_{0}$ in $B$.

Even if the coefficient is an odd integer it can still happen that "orbit (1)" is of Richardson type. In this case the only more difficult part is how to choose an invariant parabolic subalgebra. In all cases we were able to choose a polarization containing the Dynkin parabolic and here our choice could nearly always be 
made by simply taking $B_{0}$ together with a suitable subset of roots of weight 1 relative to $H$ under the heading $B_{1}$. The result is expressed under the heading "invariant parabolic", in the table, and as before $B^{\prime \prime}$ designates a $W$ conjugate in $B$.

When "orbit 1 " is not of Richardson type, we have to appeal to the information contained under the heading "degree" which is just the value of $l_{\tau\left(B^{\prime}\right) \otimes s n}$ and which by 3.10 gives a lower bound to $d\left(U(\mathfrak{g}) / J_{\max }(\lambda)\right)$. At the same time we guess a subbasis $\tilde{B}^{\prime \prime}$ of the roots such that $\left(\beta^{\vee}, \tilde{\omega}_{\alpha}\right) \in \mathbf{N}^{+}$for all $\beta \in \tilde{B}^{\prime \prime}$ and (by definition of a subbasis) there exists $w \in W$ such that $B^{\prime \prime}:=w^{-1} \tilde{B}^{\prime \prime} \subset B$. The latter defines an induced module $U(\mathfrak{g}) \otimes_{U\left(\mathfrak{p}_{B^{\prime \prime}}\right)} \mathbf{C}_{w \lambda-\rho}$ whose annihilator $J$ is contained in $J_{\max }(\lambda)$. We have $d(U(\mathfrak{g}) / J)=2 \operatorname{codim} \mathfrak{p}_{B^{\prime \prime}}$ and so if we can choose $\tilde{B}^{\prime \prime}$ such that $2 \operatorname{codim} \mathfrak{p}_{B^{\prime \prime}}=\operatorname{card} R-2 l_{\tau\left(B^{\prime}\right) \otimes s n}$ it will follow that equality holds in 3.10 and furthermore that the variety of $J_{\max }(\lambda)$ is the closure of the Richardson orbit defined by $B^{\prime \prime}$.

In each case we were in fact able to satisfy the above equality and so prove that $J_{\max }(\lambda)$ is an induced ideal. Here the choice of $\tilde{B}^{\prime \prime}$ was aided as before by a knowledge of $B_{0}$ and $B_{1}$. The resulting orbit is given under the heading "orbit (2)" and when this differs from "orbit (1)" the former was always found to be an even parabolic with $\tilde{B}^{\prime \prime}$ defining the invariant Dynkin parabolic. Thereby the above theorem was verified. The information in our table should be enough to help the energetic reader through the computation.

3.12. Fix $\mu \in P(R)^{++}$and a Duflo involution [18, III, 3.5] $\sigma$ of $W$. Let $\mathscr{C}(\sigma)$ (resp. $\mathscr{D C}(\sigma)$ ) denote the left cell (resp. double cell) containing $\sigma$ $\left[18\right.$, III, 4.6] and $m(\sigma)$ the degree of the Goldie rank polynomial $p_{\sigma w_{\mu}}$. Take $x, y \in \mathscr{D} \mathscr{C}(\sigma)$. In $[22,4.8]$ we calculated $\operatorname{Soc} L\left(L\left(x^{-1} \mu\right), L(y \mu)\right)$ though here some minor corrections had to be made [22, Corrigenda]. In more detail identify $[M(-w \rho)]$ with $w$ and define a $\mathbf{Z}$ basis $a(w): w \in W$ of $\mathbf{Z W}$ by identifying $[L(-w \rho)]$ with $a(w)$. In [22, A.3.6] we defined a bijection $w \mapsto w_{*}$ of $W$ (taking Duflo involutions to Duflo involutions and left cells to left cells) satisfying $w_{* *}=w,\left(w_{*}\right)^{-1}=\left(w^{-1}\right)_{*}$. We showed that the multiplicity of the simple module $L(M(\mu), L(z \mu))$ in $\operatorname{Soc} L(L(x \mu), L(y \mu))$ is just $(-1)^{m\left(\sigma_{*}\right)}$ times the coefficient of $a\left(z_{*}\right)$ in the truncated (circle) product $a\left(x_{*}^{-1}\right) \circ a\left(y_{*}\right)$. (This differs from the circle product on Weyl group elements (used in $\S 2$ ) and will only be used in $\S 3.13$.)

3.13. If we write

$$
a\left(x_{*}\right) \circ a\left(y_{*}\right)=\sum_{z \in W} c_{x_{*}, y_{*}, z_{*}} a\left(z_{*}^{-1}\right)
$$

then the coefficients $c_{x, y, z}$ coincide with those introduced by Lusztig (cf. [22, 3.2]). However we could also simply define $c_{x_{*}, y_{*}, z_{*}}$ to be $(-1)^{m\left(\sigma_{*}\right)}$ times the coefficient of $L\left(M(\mu), L\left(z^{-1} \mu\right)\right)$ in $\operatorname{Soc} L\left(L\left(x^{-1} \mu\right), L(y \mu)\right)$. For our present 
purposes the required properties of these coefficients can be deduced from the latter definition as indicated by the remarks in parentheses.

One has $[22,6.4]$ the relation $c_{x, y, z}=c_{y^{-1}, x^{-1}, z^{-1}}$ (which also follows from $\mathcal{O}$ duality) the property [22,3.2(iii)] that $c_{x, y, z} \neq 0$ for some $z \in W$ if and only if $x, y^{-1}$ are in the same left cell (which also follows from [11, 3.8]). It is convenient (though not necessary for our purposes) to know that the $c_{x, y, z}$ are cyclically symmetric [22, 3.2(iii)]. One has [22, 3.3, A.1]

$$
a\left(x_{*}\right) \circ a\left(\sigma_{*}\right)=(-1)^{m\left(\sigma_{*}\right)} a\left(x_{*}\right), \quad \forall x \in \mathscr{C}(\sigma)
$$

(a result equivalent to [18, III, 3.5]). Then by cyclic symmetry $a\left(\sigma_{*}\right)$ occurs with coefficient $(-1)^{m(\sigma)}$ in the decomposition of each $a\left(x_{*}\right) \circ a\left(x_{*}^{-1}\right): x \in \mathscr{C}(\sigma)$ and not in $a\left(x_{*}\right) \circ a\left(y_{*}^{-1}\right)$ for $x, y \in \mathscr{C}(\sigma)$ distinct. (This results from [33, 5.1] if we note that $L\left(L\left(x^{-1} \mu\right),\left(L\left(y^{-1} \mu\right)\right)\right.$ admits the trivial $\mathfrak{k}$ type if and only if $x=y$.) Finally a crucial property of the circle product is that it is associative [22, 3.2]. (This also follows from the associativity in the multiplication of certain completion functors [33, last paragraph of 5.10].)

3.14. Let $\mathscr{C}_{1}, \mathscr{C}_{2}$ be left cells in $\mathscr{D} \mathscr{C}(\sigma)$ which we may also view as $W$ modules. After Lusztig [27, 12.15] one has $\operatorname{dim} \operatorname{Hom}_{W}\left(\mathscr{C}_{1}, \mathscr{C}_{2}\right)=\operatorname{card}\left(\mathscr{C}_{2} \cap C_{1}^{-1}\right)$. In particular if $\mathscr{E}(\sigma)$ is an irreducible left cell then $\mathscr{C}(\sigma) \cap \mathscr{C}(\sigma)^{-1}=\{\sigma\}$ and so for all $x \in \mathscr{C}(\sigma)$ one has $\operatorname{Soc} L(L(x \mu), L(x \mu))=L(M(\mu), L(\sigma \mu))$ which is a simple module. Now take $\lambda \in P(R)^{+}$and assume that $x$ is the unique longest element in its right $W_{\lambda}^{0}$ coset. Then (cf. $[15,2.11]$ ) by translation principles $\operatorname{Soc} L(L(x \lambda), L(x \lambda))$ is also simple. Moreover if $J(x \lambda)=J_{\max }(\lambda)$ then Soc $L(L(x \lambda), L(x \lambda))=L(L(x \lambda), L(x \lambda))$ by Gelfand-Kirillov dimension estimates (essentially [18, III, 4.13]) and so the module $L(L(x \lambda), L(x \lambda))$ is simple. To apply this result we must (at all costs!) avoid having to compute $x$. For this recall $[21,3.10]$ that $\mathscr{V}(J(x \lambda))$ is the closure of a nilpotent orbit and furthermore the latter can be associated to a unique double cell $\mathscr{D} \mathscr{C}$ carrying the appropriate Springer representation as its special representation. Then it is enough to check that every left cell in $\mathscr{D C}$ is irreducible. (Except in the exceptional groups the left cells in a given double cell always have the same length.)

We shall need to generalize the above for cell length $\leq 2$. Let $\mathscr{C}_{1}$ be of length 2 (as a $W$ module). One checks from Lusztig's tables of left cells in [26, II, §11] (using [28] to ensure that the definition of cells in [26] coincides with present one) that there exists a left cell $\mathscr{C}_{2}$ with $\operatorname{dim} \operatorname{Hom}_{W}\left(\mathscr{C}_{1}, \mathscr{C}_{2}\right)=1$. Let $w$ be the unique element in $\mathscr{C}_{2} \cap \mathscr{C}_{1}^{-1}$ and $\sigma$ the Duflo involution in $\mathscr{C}_{1}$. Our assumption on $\mathscr{C}_{1}$ implies (cf. [22, 4.4]) that there is exactly one further involution $\tau \in \mathscr{C}_{1}$. Set $a=a\left(\sigma_{*}\right), b=a\left(\tau_{*}\right), x=a\left(w_{*}\right), x^{*}=a\left(w_{*}^{-1}\right)$. In the following computation we ignore the $(-1)^{m\left(\sigma_{*}\right)}$ factor which is constant on double cells and the $\circ$ sign for a circle product. 
From 3.13 one easily checks that

$$
a^{2}=a, \quad a b=b a=b, \quad b^{2}=a+k b
$$

for some $k \in \mathbf{N}$ and

$$
a x=x, \quad b x=m x, \quad x x^{*}=a+n b
$$

for some $m, n \in \mathbf{N}$ with $m \neq 0$.

Identifying coefficients of $a, b$ in the identity $b\left(x x^{*}\right)=(b x) x^{*}$ gives $m=n$ (which also follows from cyclic symmetry) and $1+k n=n m$. We conclude that $m=1$ and $k=0$.

Now choose any $u \in \mathscr{C}_{1}^{-1}$ and set $y=a\left(u_{*}\right), y^{*}=a\left(u_{*}^{-1}\right)$. We have $y y^{*}=a+r b$ for some $r \in \mathbf{N}$. Let $\mathscr{C}_{3}$ be the left cell containing $u$. Since $\mathscr{C}_{1}$ has length 2 and is multiplicity free (because the special representation occurs with multiplicity exactly one) we conclude that $\operatorname{card}\left(\mathscr{C}_{3} \cap \mathscr{C}_{1}^{-1}\right) \leq 2$.

Suppose $\operatorname{card}\left(\mathscr{C}_{3} \cap \mathscr{C}_{1}^{-1}\right)=1$. Then we must have $b y=s y$ for some $s \in \mathbf{N}^{+}$ and our previous analysis shows that $r=s=1$.

Suppose $\operatorname{card}\left(\mathscr{C}_{3} \cap \mathscr{C}_{1}^{-1}\right)=2$ and let $v$ denote the second element in $\mathscr{C}_{3} \cap$ $\mathscr{C}_{1}^{-1}$. Set $z=a\left(v_{*}\right), z^{*}=a\left(v_{*}^{-1}\right)$. We have $b y=s y+t z$ for some $s, t \in \mathbf{N}$ with $s+t>0$. Yet $b^{2}=a$ and so by $(*)$ we obtain $y=s b y+t b z$. Since $b z$ is also nonzero and a linear combination of $y, z$ with coefficients $\geq 0$ we conclude that $s+t=1$ (after a suitable interpretation of $b^{2}=a$ this also follows from [18, III, 2.16(i)]). Now $y z^{*} \neq 0$ and $a$ cannot occur in this product so we have $y z^{*}=l b: l \in \mathbf{N}^{+}$. Then $(b y) z^{*}=l b^{2}=l a^{2}$. Since again $a$ cannot occur in $y z^{*}$ we conclude that $b y=z$, that is $s=0, t=1$. Equating the coefficient of $a$ in $b\left(y y^{*}\right)=(b y) y^{*}$ (or by cyclic symmetry) we conclude that $r=0$.

The above result may be summarized as follows. Let $\mathscr{C}$ be a left cell of length 2. Then for each $w \in \mathscr{C}$ the length of Soc $L(L(w \mu), L(w \mu))$ is less than or equal to 2 and equals 2 exactly if the right cell $\mathscr{C}^{\prime}$ containing $w$ satisfies $\mathscr{C} \cap \mathscr{C}^{\prime}=\{w\}$. In particular take $\lambda \in P(R)^{+}$and let $M$ be a simple highest weight module satisfying Ann $M=J_{\max }(\lambda)$. Let $\mathscr{D} \mathscr{C}$ denote the double cell associated to $J_{\max }(\lambda)$ as described above. Then

Lemma. Suppose that every left cell in $\mathscr{D} \mathscr{C}$ has length 1 (resp. length $\leq 2$ ). Then $L(M, M)$ has the length 1 (resp. length $\leq 2$ ).

3.15. Retain the hypotheses of the above lemma. If we are in the case of length 1 , it is clear that $U(\mathfrak{g}) / \operatorname{Ann} M=L(M, M)$. Now suppose we are in the length 2 case. Assume that we can find a second simple highest weight module $M^{\prime}$ with Ann $M^{\prime}=J_{\max }(\lambda)$.

Corollary. Suppose $z:=\operatorname{rk} L(M, M) / \operatorname{rk} L\left(M^{\prime}, M^{\prime}\right)>1$. Then $U(\mathfrak{g}) / J_{\max }(\lambda)$ $=L\left(M^{\prime}, M^{\prime}\right)$ and $z=2$. 
Write $M=L\left(w^{\prime} \lambda\right), M^{\prime}=L\left(w^{\prime \prime} \lambda\right)$ with $w^{\prime}, w^{\prime \prime}$ the longest elements in their right $W_{\lambda}^{0}$ cosets. As in [18, I, 5.12(iii)] we define

$$
z_{w}=\frac{\operatorname{rk} L(L(w \mu), L(w \mu))}{\operatorname{rk}(U(\mathfrak{g}) / J(w \mu))}
$$

for each $w \in W, \mu \in P(R)^{+}$with $w$ the longest element in its right $W_{\mu}^{0}$ coset. By $[22,5.8]$ we have (making suitable corrections!) that

$$
z_{x^{-1}} z_{y^{-1}}=\sum_{w \in W}(-1)^{m\left(\sigma_{*}\right)} c_{x_{*}, y_{*}, w *} z_{w}
$$

for all $x, y \in \mathscr{D} \mathscr{C}(\sigma)$. Now $z_{\sigma}=1[18, \mathrm{I}, 3.4]$ and so in the above situation the relation $b^{2}=a$ implies $z_{\tau}^{2}=z_{\sigma}=1$. Hence $z_{\tau}=1$. Take $w \in C(\sigma)$ and let $\mathscr{C}^{\prime}$ be the right cell containing $w$. If $\operatorname{card}\left(\mathscr{C}(\sigma) \cap \mathscr{C}^{\prime}\right)=2$ then $z_{w} z_{w^{-1}}=z_{\sigma}=1$ and so $z_{w}=z_{w^{-1}}=1$. When $w=w^{\prime \prime}$ this contradicts the hypothesis $z>1$. If $\operatorname{card}\left(\mathscr{C}(\sigma) \cap \mathscr{C}^{\prime}\right)=1$ then $z_{w} z_{w^{-1}}=z_{\sigma}+z_{\tau}=2$. When $w=w^{\prime}$ the hypothesis $z>1$ forces $z_{w^{\prime}}=2, z=2$ and $z_{w^{\prime \prime}}=1$. Finally $w^{\prime}, w^{\prime \prime}$ are in the same left cell (because $M, M^{\prime}$ have the same annihilator) and so we have

$$
z_{w^{\prime \prime}-1} z_{w^{\prime}}=\sum_{w \in \Gamma}(-1)^{m(\sigma)} c_{w_{*}^{\prime \prime}, w_{*}^{\prime-1}, w *} z_{w^{*}}
$$

where $\Gamma=\mathscr{C}^{\prime} \cap C^{\prime \prime-1}$ with $\mathscr{C}^{\prime}$ (resp. $\mathscr{C}^{\prime \prime}$ ) the right cell containing $w^{\prime}$ (resp. $\left.w^{\prime \prime}\right)$. By the above if $\operatorname{card} \Gamma=2$ (resp. $\operatorname{card} \Gamma=1$ ) then $z_{w}=1$ (resp. $x_{u} \leq 2$ ) for all $w \in \Gamma$. By straightforward manipulations (involving the circle product) of the type described in 3.14 one checks that the coefficients $(-1)^{m(\sigma)} c_{w^{\prime \prime}, w_{*}^{\prime-1}, w^{*}} \leq 1$. We conclude that $z_{w^{\prime \prime-1}}=1$. Recalling [18, III, 3.4 and $19,9.1]$ the corollary results.

3.16. We shall apply 3.15 to the case when $L(w \lambda)$ is a module induced from a finite dimensional simple module $V$ of a parabolic subalgebra $\mathfrak{p}_{B^{\prime}}$ (in short a module induced from $\left.\mathfrak{p}_{B^{\prime}}\right)$. In this case $L(L(w \lambda), L(w \lambda)$ ) identifies with a generalized principal series module. For our analysis we must be able to calculate the multiplicity of $\mathfrak{k}$ types in such a module. For this we proceed as follows. Recall that as a $U\left(\mathfrak{p}_{B^{\prime}}\right)$ module $V$ is completely determined by its highest weight say $\mu-\rho$ (one must have $\mu=w \lambda$ if $L(w \lambda) \cong U(\mathfrak{g}) \otimes_{U\left(\mathfrak{p}_{B^{\prime}}\right)} V$ ). Choose $-\nu \in P(R)^{++}$such that $\left(\alpha^{\vee}, w_{B^{\prime}} \nu\right)=\left(\alpha^{\vee}, \mu\right)$ for all $\alpha \in B^{\prime}$. Let $E$ be a finite dimensional simple $\mathfrak{g}$ module and $E_{\delta}$ its $\mathfrak{h}$ weight subspace of weight $\delta$.

Lemma. Assume that $L(\mu)$ is a module induced from $\mathfrak{p}_{B^{\prime}}$. Then

$$
[L(L(\mu), L(\mu)): E]=\sum_{w \in W_{B^{\prime}}^{\prime}}(-1)^{l(w)} \operatorname{dim} E_{\mu-w^{\prime} \mu} .
$$

Since $L(\mu)$ is self-dual in the $\mathfrak{O}$ category we have by a standard isomorphism that $L(L(\mu), L(\mu))$ identifies with $L\left((L(\mu) \otimes L(\mu))^{*}\right)$ and hence with a 
generalized principal series module. Then $\left[L\left((L(\mu) \otimes L(\mu))^{*}\right): E\right]$ can be calculated by Frobenius reciprocity (cf. $[9,2.12,6.3])$ and one easily checks that the result depends only on the values of $\left(\mu, \alpha^{\vee}\right): \alpha \in B^{\prime}$. In particular we can choose $\mu=w_{B^{\prime}} \nu$ with $-\nu \in P(R)^{++}$. In this case the above formula follows immediately from $[11,4.4,5.3]$ if we note that $\operatorname{dim} E_{\nu-w \nu}=\operatorname{dim} E_{\mu-w \mu}$ when $\mu=w_{B^{\prime}} \nu+\delta$ given that $(\delta, \alpha)=0$ for all $\alpha \in B^{\prime}$. This proves the assertion.

3.17. The superiority of 3.16 over the Frobenius reciprocity formula is indicated by the following results needed in computing our table. Here $E_{\text {ad }}$ denotes the adjoint representation and in the case when $\mathfrak{g}$ has roots of different lengths we let $E_{s}$ denote the representation generated by a short root vector and $B_{s}$ the set of short simple roots.

Corollary. Take $\mu \in \mathfrak{h}^{*}$ and assume that $L(\mu)$ is a module induced from $\mathfrak{p}_{B^{\prime}}$. Then

$$
\begin{aligned}
& {\left[L(L(\mu), L(\mu)): E_{\mathrm{ad}}\right]=\operatorname{rank} \mathfrak{g}-\operatorname{card}\left\{\alpha \in B^{\prime} \mid\left(\alpha^{\vee}, \mu\right)=1\right\} .} \\
& {\left[L(L(\mu), L(\mu)): E_{s}\right]=\operatorname{dim} E_{s}^{\mathfrak{h}}-\operatorname{card}\left\{\alpha \in B^{\prime} \cap B_{s} \mid\left(\alpha^{\vee}, \mu\right)=1\right\} .}
\end{aligned}
$$

In 3.16 we may assume that $\mu \in P(R)^{++}$without loss of generality. Then we can show that the only nonzero contributions in the sum come when $l(w) \leq 1$. In fact this follows from say $[23,3.1]$ and furthermore if $w=s_{\alpha}: \alpha \in B^{\prime}$ we must have $\left(\alpha^{\vee}, \mu\right)=1$. Since the dimension of the zero weight space for the adjoint representation is just rank $g$ and every root space occurs with multiplicity one, this gives (i). (ii) follows similarly.

3.18. We shall need the following technical result. Let $\mathfrak{g}$ be simple of type $B_{n}$ and let $\alpha$ be the unique short root of $B$. Let $B_{1}, B_{2}$ be subsets of $B$ having the same cardinality (in our eventual application of the same type) and assume $\alpha \in B_{1}$ but $\alpha \notin B_{2}$. Let $\mu_{1}, \mu_{2} \in \mathfrak{h}^{*}$ satisfy $\left(\mu_{1}, \alpha^{\vee}\right)=2,\left(\mu_{1}, \beta^{\vee}\right)=1$, for all $\beta \in B_{1}-\{\alpha\} ;\left(\mu_{2}, \alpha^{\prime \vee}\right)=2$ for some $\alpha^{\prime} \in B_{2}$ and $\left(\mu_{2}, \beta^{\vee}\right)=1$ for all $\beta \in B_{2}-\{\alpha\}$. Let $E_{2 s}$ denote the simple finite dimensional $U(\mathfrak{g})$ module with extreme weight $2 a$.

Lemma. Assume that $L\left(\mu_{i}\right)$ is a module induced from $\mathfrak{p}_{B^{\prime}}: i=1,2$. Then

$$
\left[L\left(L\left(\mu_{1}\right), L\left(\mu_{1}\right)\right): E_{2 s}\right]=\left[L\left(L\left(\mu_{2}\right), L\left(\mu_{2}\right)\right): E_{2 s}\right]-1 .
$$

As before we can assume $\mu_{i} \in P(R)^{++}: i=1,2$. Then one easily shows that the above formula results if one can show that there are no contributions coming from terms for which $l(w) \geq 2$ in the right-hand side of 3.16. Here to obtain such a nonzero contribution we must have

$$
(\mu-w \mu, \mu-w \mu) \leq(2 \alpha, 2 \alpha)=4(\alpha, \alpha) .
$$

Now take $\gamma \in B$ such that $l\left(s_{\gamma} w\right)>l(w)$ (equivalently that $w^{-1} \gamma \in R^{+}$). One has

$$
\begin{aligned}
& \left(\mu-s_{\gamma} w \mu, \mu-s_{\gamma} w \mu\right)-(\mu-w \mu, \mu-w \mu) \\
& \quad=2\left(w \mu-s_{\gamma} w \mu, \mu\right)=2\left(w \mu, \gamma^{\vee}\right)(\gamma, \mu)>0
\end{aligned}
$$


and so it is enough to show that for $l(w)=2$ we can at best get equality in $(*)$ and to analyze this case. Let us write $w=s_{\gamma} s_{\delta}$ with $\gamma, \delta \in B_{1}$ or $B_{2}$. The previous analysis shows that we can assume $\left(\delta^{\vee}, \mu\right)=1$ so $\delta$ is not short and replacing $w$ by $w^{-1}$ that both $\gamma, \delta$ are long. Then $(\mu-w \mu, \mu-$ $w \mu)=\left(\mu, \delta^{\vee}\right)(\delta, \delta)+2(\gamma, \mu)\left(\mu\left(s_{\delta} \gamma\right)^{\vee}\right)=2(\alpha, \alpha)+2\left(\mu,\left(s_{\delta} \gamma\right)^{\vee}\right)(\alpha, \alpha) \geq 4(\alpha, \alpha)$ with equality only if $(\delta, \gamma)=0$. However the sum of two orthogonal long simple roots is not twice a short root hence not a weight of $E_{2 s}$, so we get zero contribution from these cases.

3.19. The simplicity of the induced module is not essential to obtain an embedding in a generalized principal series module. Indeed we have the

Lemma. Take $M \in \mathrm{Ob} \mathfrak{O}$. If $N:=\operatorname{Soc} M$ is simple and $d(M / N)<d(M)$ then we have an embedding $U(\mathfrak{g}) / \operatorname{Ann} M$ into $L\left((M \otimes M)^{*}\right)$.

Let $\delta$ denote the duality functor on $\mathfrak{O}$. A standard isomorphism restricts to an isomorphism of $L(\delta M, M)$ onto $L\left((M \otimes M)^{*}\right)$.

The exact sequence $0 \rightarrow N \rightarrow M \rightarrow M / N \rightarrow 0$ gives exact sequences

$$
0 \rightarrow L(N, N) \rightarrow L(N, M) \rightarrow L(N, M / N) \rightarrow
$$

and

$$
\leftarrow L(N, M) \leftarrow L(M, M) \leftarrow L(M / N, M) \leftarrow 0 .
$$

Under the hypothesis of the lemma $[16,8.16,8.17]$ implies that $L(N, M / N)=0$ and $L(M / N, M)=0$ and so we get an embedding of $L(M, M)$ into $L(N, N)$.

Since $\delta N \cong N$ we also have an exact sequence $0 \rightarrow \delta(M / N) \rightarrow \delta M \rightarrow N \rightarrow$ 0 . As before $L(\delta(M / N), N)=0$ and so we obtain an isomorphism

$$
L(N, N) \stackrel{\sim}{\longrightarrow} L(\delta M, N) .
$$

Finally the embedding of $N$ into $M$ gives an embedding of $L(\delta M, N)$ into $L(\delta M, M)$ and proves the lemma.

3.20. Take $B^{\prime} \subset B$ and $\mu \in \mathfrak{h}^{*}$ such that $\left(\mu, \alpha^{\vee}\right) \in \mathbf{N}^{+}$for all $\alpha \in B^{\prime}$. Then we have a finite dimensional simple $U\left(\mathfrak{p}_{B^{\prime}}\right)$ module $V_{B^{\prime}}(\mu)$ with highest weight $\mu-\rho$. Set $M_{B^{\prime}}(\mu)=U(\mathfrak{g}) \otimes_{U\left(\mathfrak{p}_{B^{\prime}}\right)} V_{B^{\prime}}(\mu)$. Let $\mathscr{O}_{B^{\prime}}$ denote the Richardson orbit defined by $B^{\prime}$. The following result was noted by Gupta $[12,2.8]$. We sketch a proof for completion.

Lemma. Suppose $\mathfrak{p}_{B^{\prime}}$ is an invariant parabolic subalgebra, $\operatorname{dim} V_{B^{\prime}}(\mu)=1$ and $\mathscr{O}_{B^{\prime}}$ has normal closure. Then the map $U(\mathfrak{g}) / \operatorname{Ann} M_{B^{\prime}}(\mu) \rightarrow L\left(M_{B^{\prime}}(\mu), M_{B^{\prime}}(\mu)\right)$ is an isomorphism. Furthermore $\left[U(\mathfrak{g}) / \operatorname{Ann} M_{B^{\prime}}(\mu): E\right]=E^{\mathfrak{r}^{B^{\prime}}}$ for any finite dimensional $\mathfrak{g}$ module $E$.

It is well known that $M=M_{B^{\prime}}(\mu)$ with $\operatorname{dim} V_{B^{\prime}}(\mu)=1$ satisfies the hypothesis of 3.19. Since $\left[L\left(\left(M_{B^{\prime}}(\mu) \otimes M_{B^{\prime}}(\mu)\right)^{*}\right): E\right]=\operatorname{dim} E^{\mathfrak{r}_{B^{\prime}}}$ by Frobenius reciprocity we obtain $\left[U(\mathfrak{g}) / \operatorname{Ann} M_{B^{\prime}}(\mu): E\right] \leq \operatorname{dim} E^{\mathfrak{r}_{B^{\prime}}}$. Set $X=G / P_{B^{\prime}}$ and $I_{X}=\operatorname{Ann} M_{B^{\prime}}\left(-w_{B^{\prime}} \rho\right)$. If $M=M_{B^{\prime}}\left(-w_{B^{\prime}} \rho\right)$ then by [9, combine 2.12, 4.7, 
$5.5,6.13]$ the embedding in 3.19 is an isomorphism. In the language of BorhoBrylinski, $I_{X}$ is the annihilator of the operator representation of the generalized flag variety $X$. They show $[5,5.6]$ that $\operatorname{gr} I_{X}=\sqrt{\operatorname{gr} I_{X}}$ under the hypothesis of the lemma. Yet $\sqrt{\operatorname{gr} I_{X}}=\sqrt{\operatorname{grAnn} M_{B^{\prime}}(\mu)}$ by $[5,4.7]$. Then

$$
\begin{aligned}
\operatorname{dim} E^{\mathfrak{r}_{B^{\prime}}} & =\left[U(\mathfrak{g}) / I_{X}: E\right]=\left[S(\mathfrak{g}) / \operatorname{gr} I_{X}: E\right]=\left[S(\mathfrak{g}) / \sqrt{\operatorname{gr} I_{X}}: E\right] \\
& =\left[S(\mathfrak{g}) / \sqrt{\operatorname{gr} \operatorname{Ann} M_{B^{\prime}}(\mu)}: E\right] \leq\left[U(\mathfrak{g}) / \operatorname{Ann} M_{B^{\prime}}(\mu): E\right] \leq \operatorname{dim} E^{\mathfrak{r}_{B^{\prime}}},
\end{aligned}
$$

which (cf. 3.19) proves the required assertions.

3.21. It may be a nuisance to verify the last condition of 3.20 and in fact when Ann $M_{B^{\prime}}(\mu)$ is a maximal ideal we do not even need it. Actually it is educative to observe the following more general result. Assume that $\operatorname{dim} V_{B^{\prime}}(\mu)=1$. Then (cf. [16, 15.21(2)]) $L\left(M_{B^{\prime}}(\mu), M_{B^{\prime}}(\mu)\right)$ is an integral domain admitting $U(\mathfrak{g}) / \operatorname{Ann} M_{B^{\prime}}(\mu)$ as a subring. Since both rings are noetherian they admit classical rings of fractions and we obtain an embedding [16, 11.16] Fract $U(\mathfrak{g}) / \operatorname{Ann} M_{B^{\prime}}(\mu) \hookrightarrow$ Fract $L\left(M_{B^{\prime}}(\mu), M_{B^{\prime}}(\mu)\right)$ of skew-fields. Let $\tilde{z}_{B^{\prime}}$ denote the dimension of a larger over the smaller which (see below) is the same on either side. Take $X \in \mathscr{O}_{B^{\prime}}$ and recall the notation of 3.11.

Lemma. $\tilde{z}_{B^{\prime}}$ divides $\left[G^{X}: P_{B^{\prime}}^{X}\right]$.

In the language of $[4,5.8]$ let $y$ denote the $p$-multiplicity of $\operatorname{Ann} M_{B^{\prime}}(\mu)$. It is enough to show that $y \tilde{z}_{B^{\prime}}=\left[G^{X}: P_{B^{\prime}}^{X}\right]$. If $M$ is a finitely generated left $U(\mathfrak{g})$ module let $e(M)$ denote its Bernstein multiplicity. One has

$$
\tilde{z}_{B^{\prime}}=e\left(L\left(M_{B^{\prime}}(\mu), M_{B^{\prime}}(\mu)\right)\right) / e\left(U(\mathfrak{g}) / \operatorname{Ann} M_{B^{\prime}}(\mu)\right)
$$

by $[16,11.20]$. Since $M_{B^{\prime}}(\mu), M_{B^{\prime}}\left(-w_{B^{\prime}} \rho\right)$ are isomorphic as $U\left(\mathfrak{n}^{+}\right)$modules, $\left(M_{B^{\prime}}(\mu) \otimes M_{B^{\prime}}(\mu)\right)^{*},\left(M_{B^{\prime}}\left(-w_{B^{\prime}} \rho\right) \otimes M_{B^{\prime}}\left(-w_{B^{\prime}} \rho\right)\right)^{*}$ are isomorphic as $U\left(\mathfrak{n}^{+}\right) \otimes U\left(\mathfrak{n}^{+}\right)$modules. By Frobenius reciprocity (cf . [10, 5.5.8]) they are isomorphic as $U(\mathfrak{k})$ modules and so $L\left(\left(M_{B^{\prime}}(\mu) \otimes M_{B^{\prime}}(\mu)\right)^{*}\right), L\left(\left(M_{B^{\prime}}\left(-w_{B^{\prime}} \rho\right) \otimes\right.\right.$ $\left.\left.M_{B^{\prime}}\left(-w_{B^{\prime}} \rho\right)\right)^{*}\right)$ are isomorphic as $U\left(\mathfrak{n}^{+}\right) \otimes U\left(\mathfrak{n}^{+}\right)$modules. Now as noted in $[18$, II, 5.6] their Bernstein multiplicities may be calculated from just this latter structure and so we conclude that

$$
\begin{aligned}
e\left(L\left(M_{B^{\prime}}(\mu), M_{B^{\prime}}(\mu)\right)\right) & =e\left(L\left(\left(M_{B^{\prime}}(\mu) \otimes M_{B^{\prime}}(\mu)\right)^{*}\right)\right) \\
& =e\left(L\left(\left(M_{B^{\prime}}\left(-w_{B^{\prime}} \rho\right) \otimes M_{B^{\prime}}\left(-w_{B^{\prime}} \rho\right)\right)^{*}\right)\right)=e\left(U(\mathfrak{g}) / I_{X}\right) .
\end{aligned}
$$

(Here the first equality holds because the embedding defined by 3.19 with $M=M_{B^{\prime}}(\mu)$ can fail to be an isomorphism only by terms coming from Ext groups involving $M / N$. Because $d(M / N)<d(M)$ these have strictly lower Gelfand-Kirillov dimension and the last equality follows from the Berline-Duflo isomorphism noted in 3.20.) Taking account of $[4,5.8,5.9,5.11 ; 6,3.9]$ and recalling (3.20) that $\sqrt{\mathrm{gr} I_{X}}=\sqrt{\mathrm{gr} M_{B^{\prime}}(\mu)}$ gives the required assertion.

3.22. (Notation $3.17,3.20$.) The above result gives the following version of 3.20 , 
namely

Theorem. Suppose $\mathfrak{p}_{B^{\prime \prime}}$ is an invariant parabolic subalgebra, $\operatorname{dim} V_{B^{\prime \prime}}(\mu)=1$ and Ann $M_{B^{\prime \prime}}(\mu)=J_{\max }(\mu)$. Then $U(\mathfrak{g}) / \operatorname{Ann}_{B^{\prime \prime}}(\mu)$ is a generalized principal series module. In particular

$$
\begin{gathered}
{\left[J_{\max }(\mu) / J_{\min }(\mu): E_{\mathrm{ad}}\right]=\operatorname{card} B^{\prime \prime} .} \\
{\left[J_{\max }(\mu) / J_{\min }(\mu): E_{s}\right]=\operatorname{card} B^{\prime \prime} \cap B_{s} .}
\end{gathered}
$$

By 3.21 the hypothesis implies that $\tilde{z}_{B^{\prime}}=1$. Then the first part follows as in say $[19,9.1(\mathrm{ii})]$. The second part follows from 3.17 .

3.23. Take $\lambda \in \mathfrak{h}^{*}$ and $w \in W_{\lambda}$ satisfying $w=w_{\lambda}^{0}$ (that is $w$ is the unique maximal longest element in its right $W_{\lambda}^{0}$ coset). In [18, III, 2.8, 3.4] we associated two integers $z_{w}, \tilde{z}_{w}$ to the embedding $U(\mathfrak{g}) / J(w \lambda) \hookrightarrow L(L(w \lambda), L(w \lambda))$. The first measures the ratio of Goldie ranks and the second generalizes the $\tilde{z}_{B^{\prime}}$ defined in 3.21. Curiously $\tilde{z}_{w}=z_{w^{-1}}[18$, III, 3.4]. Let $G X$ denote the nilpotent orbit whose closure is $\mathscr{V}(J(w \lambda))$-recall [21, 3.10] and let $A_{X}$ denote the component group of $G^{X}$. One may ask if $z_{w}$ divides $\left|A_{X}\right|$. A difficulty here is that 3.21 fails to extend to the case when $z_{w}>1$. For example take $w=s_{\beta} s_{\alpha} s_{\beta}$ and $\lambda=\bar{\omega}_{\alpha}$ in type $G_{2}$. By say 5.4 one has $L(w \lambda)=M_{\alpha}(w \lambda)$. Yet $z_{w}, \tilde{z}_{w}=2$ [18, III, Table II] which divide $\left|A_{X}\right|=6$ [8, p. 401]; but not $\left[G^{X}: P_{\alpha}^{X}\right]$ which equals 1 (resp. 3) if $\alpha$ is short (resp. long)-see $[4,5.7]$.

3.24. We need the following technical result. Assume $\mathfrak{g}$ simple of type $F_{4}$ and take $B=\left\{\alpha_{1}, \alpha_{2}, \alpha_{3}, \alpha_{4}\right\}$ with $\alpha_{3}, \alpha_{4}$ short. Set $s_{\alpha_{i}}=s_{i}: i=1,2,2,4$ and $x=s_{4} s_{2} s_{1} s_{2}, y=s_{4} s_{2} s_{1} s_{2} s_{3}, z=s_{4} s_{2} s_{1} s_{2} s_{3} s_{2}, \sigma_{1}=s_{3} s_{4} s_{3} s_{1}, \sigma_{2}=s_{2} \sigma_{1} s_{2}$, $\sigma_{3}=s_{3} \sigma_{2} s_{3}, u=s_{2} \sigma_{1}, v=s_{3} \sigma_{2}$. Observe that $w_{B}=-1$.

Lemma. For all $\xi \in-P(R)^{++}$one has

$$
\begin{aligned}
\operatorname{Soc} L(L(x \xi), L(x \xi)) & =V\left(\sigma_{3} \xi, \xi\right) . \\
\operatorname{Soc} L(L(x \xi), L(y \xi)) & =V(v \xi, \xi) . \\
\operatorname{Soc} L(L(y \xi), L(y \xi)) & =V\left(\sigma_{2} \xi, \xi\right) . \\
\operatorname{Soc} L(L(y \xi), L(z \xi)) & =V(u \xi, \xi) . \\
\operatorname{Soc} L(L(z, \xi), L(z \xi)) & =V\left(\sigma_{1} \xi, \xi\right) .
\end{aligned}
$$

This follows from $[11,4.4 ; 18$, III, 4.2, 5.5; 5, 2.17c] and the table. In fact recall the Borho-Jantzen-Duflo $\tau$-invariant defined by first setting $\tau(w)=$ $B \cap w^{-1} R^{-}$. In general $L(z \xi)$ is a quotient of $M_{B^{\prime \prime \prime}}(z \xi)$ where $B^{\prime \prime \prime}=\tau\left(z^{-1}\right)=$ $\left\{\alpha_{1}, \alpha_{2}, \alpha_{4}\right\}$ from which we conclude that

$$
d(L(z \xi)) \leq d\left(M_{B^{\prime}}(z \xi)\right)=\operatorname{card} R^{+}-\operatorname{card} R^{\prime \prime \prime+}=\operatorname{card} R^{+}-4 .
$$

Yet $\tau(z)=\left\{\alpha_{2}\right\}$ and from the heading "degree" in the table for $\alpha=\alpha_{2}$ in type $F_{4}$ we conclude that $d\left(L\left(-w_{B^{\prime}} \xi\right)\right)=\operatorname{card} R^{+}-4$ where $B^{\prime}=\left\{\alpha_{1}, \alpha_{3}, \alpha_{4}\right\}$. 
Then by $[5,2.17 \mathrm{c}]$ we obtain $J(z \xi)=J\left(-w_{B^{\prime}} \xi\right)$. Yet $\sigma_{1}=w_{B^{\prime}}$ and by [18, III, 4.2] the latter is a Duflo involution and we conclude that $V\left(\sigma_{1} \xi, \xi\right)=$ $\operatorname{Soc}(U(\mathfrak{g}) / J(z \xi))$ which is hence a simple submodule of $L(L(z \xi), L(z \xi))$.

Now we use [18, III, 5.5] to prove successively that the right-hand sides of (v)-(i) occur as simple submodules of the left-hand sides. Here one should note in particular the behavior of the $\tau$-invariant. Yet by $[11,4.4]$ the right-hand side of (i) is just $\operatorname{Soc} U(\mathfrak{g}) / J(x \xi)$ which is a simple module. This proves (i) and shows in particular that $\sigma_{3}$ is a Duflo involution. Finally we apply [18, III, 5.5] to prove successively (i)-(v).

3.25. Retain the notation and hypotheses of 3.24. Set $\alpha=\alpha_{2}$. Using Jantzen's irreducibility criterion (5.4) one checks that $M_{B^{\prime \prime \prime}}\left(-z \bar{\omega}_{\alpha}\right)$ is a simple module and hence equals $L\left(-z \bar{\omega}_{\alpha}\right)$. Noting that $z$ is of minimal length in its right $W_{\xi}^{0}$ coset $\left(\xi=-\bar{\omega}_{\alpha}\right)$ we conclude by translation principles and $3.24(\mathrm{v})$ that $U(\mathfrak{g}) / J_{\max }\left(\bar{\omega}_{\alpha}\right)$ coincides with $L\left(M_{B^{\prime \prime \prime}}\left(-z \bar{\omega}_{\alpha}\right), M_{B^{\prime \prime \prime}}\left(-z \bar{\omega}_{\alpha}\right)\right)$ which is a (generalized) principal series module. Also $\tilde{B}^{\prime \prime \prime}:=-z^{-1} B^{\prime \prime \prime}=\left\{\alpha_{2}+2 \alpha_{3}, \alpha_{1}+\right.$ $\left.\alpha_{2}, \alpha_{2}+\alpha_{3}+\alpha_{4}\right\}$ and so $\operatorname{dim} V_{B^{\prime \prime \prime}}\left(-z \bar{\omega}_{\alpha}\right)=2$.

\section{MULTIPLiCity ON CORNERS}

4.1. Fix $\alpha \in B$ and set $\lambda=\bar{\omega}_{\alpha}$. Let $\bar{\alpha}$ denote the root affiliated to $\alpha$ as defined in $[23,4.1]$. By $[23,2.4,4.1]$ the adjoint representation occurs with multiplicity one in $V\left(-\lambda,-s_{\bar{\alpha}} \lambda\right)$ and does not occur in any other simple subquotient of $J_{\max }(\lambda) / J_{\min }(\lambda)$. (A little care is needed to obtain the above conclusion because $\lambda$ had previously been taken to be antidominant.) This gives the

Lemma. Take $\lambda=\bar{\omega}_{\alpha}$. Then

$$
\left[U(\mathfrak{g}) / J_{\min }(\lambda): V\left(-\lambda,-s_{\bar{\alpha}} \lambda\right)\right]=\left[J_{\max }(\lambda) / J_{\min }(\lambda): E_{\mathrm{ad}}\right] .
$$

Remark. The left-hand side is of course independent of the choice of $\lambda$ on the $\alpha$ corner.

4.2. We attempt to calculate the right-hand side of 4.1 by using 3.22 . By 3.11 all the required hypotheses are satisfied except possibly that $\operatorname{dim} V_{B^{\prime \prime}}(\mu)=1$ which may fail. (Here $\mu=w \lambda$ where $w \in W$ conjugates the subbasis $\tilde{B}^{\prime \prime}$ of $R^{+}$listed in the table under the heading "invariant parabolic" into $B^{\prime \prime}$ - see also the discussion in the proof of 3.11.) We list $\operatorname{dim} V_{B^{\prime \prime}}(\mu)$ for our particular choices in the table and when $\operatorname{dim} V_{B^{\prime \prime}}(\mu)=1$ we obtain the left-hand side of 4.1 to be card $B$ " and this is listed under the heading "multiplicity" in the table.

4.3. Suppose now that $\operatorname{dim} V_{B^{\prime \prime}}(\mu)>1$ (as listed). When root lengths differ we can sometimes avoid the ensuing difficulty by passing to the "Langlands' dual" of $\mathfrak{g}$. Indeed recalling that $\lambda$ is integral it follows by the truth of the Kazhdan-Lusztig conjectures that the left-hand side of 4.1 depends only on the specification of the pair $(W, B)$ as a Coxeter gioup. For example, take $\mathfrak{g}$ of type $B_{n}$ with $\alpha=\alpha_{2 m+1}: 3 m+1<n$. Then $\operatorname{dim} V_{B^{\prime \prime}}(\mu)=2$. Since $\alpha$ is long we have $\alpha=\bar{\alpha}$. By the above remark we may calculate $\left[U(\mathfrak{g}) / J_{\min }(\lambda)\right.$ : 
$\left.V\left(-\lambda,-s_{\alpha} \lambda\right)\right]$ by assuming that the data refers to $\mathfrak{g}$ of type $C_{n}$. Now in this case it happen fortuitously (see table) that $\operatorname{dim} V_{B^{\prime \prime}}(\mu)=1$. However $\alpha$ is now a short root in type $C_{n}$ (and in fact distinct from the affiliated root) so the result is not necessarily the multiplicity in type $C_{n}$ (which equals $2 m+1$ ). In fact the required value is given by $\left[J_{\max }(\lambda) / J_{\min }(\lambda): E_{s}\right]$ and by 3.22 (ii) equals $\operatorname{card}\left(B^{\prime \prime} \cap B_{s}\right)=2 m+1$.

4.4. A second and more interesting case occurs in type $C_{n}$ with $\alpha=\alpha_{n}$. Then $\operatorname{dim} V_{B^{\prime \prime}}(\mu)=2^{[n / 2]}$. Since $\alpha$ is long we have $\alpha=\bar{\alpha}$. As before we calculate $\left[U(\mathfrak{g}) / J_{\min }(\lambda): V\left(-\lambda,-s_{(} \lambda\right)\right]$ by assuming that the data refers to $\mathfrak{g}$ of type $B_{n}$. Here again $\operatorname{dim} V_{B^{\prime \prime}}(\mu)=1$ (see table). However $\alpha$ is now a short root in type $B_{n}$ (and in fact distinct from the affiliated root) so the result is not necessarily the multiplicity in type $B_{n}$ (which equals $\left.[(n+1) / 2]\right)$. Indeed the required value is given by $\left[J_{\max }(\lambda) / J_{\min }(\lambda): E_{s}\right]$ and by 3.22 (ii) equals $\operatorname{card}\left(B^{\prime \prime} \cap B_{s}\right)=1$.

4.5. In the above situation we observe that the multiplicity is much smaller than we might expect and more precisely strictly smaller than card $B^{\prime \prime}=[(n+1) / 2]$. This could lead to a third way in which Gupta's theorem can fail for "special" $\lambda$. The first bad case occurs for $n=3$. With $\alpha=\alpha_{3}$ and $\lambda=\bar{\omega}_{3}$ we find that $J_{\max }(\lambda) / J_{\min }(\lambda)$ admits only one copy of the adjoint representation. Let us show that this cannot generate $J_{\max }(\lambda)$. In fact from the heading "degree" we conclude that $d\left(U(\mathfrak{g}) / J_{\max }(\lambda)\right)=\operatorname{card} R-4$. On the other hand $V:=V_{\alpha_{3}}(\lambda)$ is a one dimensional representation of the almost minimal parabolic subalgebra $\mathfrak{p}:=\mathfrak{p}_{\alpha_{3}}$ and so the corresponding induced ideal $I:=\operatorname{Ann} U(\mathfrak{g}) \otimes_{U(\mathfrak{p})} V$ is contained in $J_{\max }(\lambda)$ (strictly because $d(U(\mathfrak{g}(/ I)=$ card $R-2)$. By [23, 2.4] the adjoint representation already occurs in $I / J_{\min }(\lambda)$. However we do not get a contradiction to Gupta's theorem because although $J_{\max }(\lambda)$ is induced by 3.4 , it is not completely prime. This will follow from $3.14,[16,15.2(2)]$ and the fact that $\operatorname{dim} V_{B^{\prime \prime}}(\lambda)=2$ if we can show that the corresponding left cell is irreducible. According to the column "orbit (2)" the zero variety of $J_{\max }(\lambda)$ is the closure of the orbit $(3 ; \varnothing)$ in Carter's notation-the elementary divisors being 3,3. According to Lusztig [26, I, p. 334] this orbit (which is Richardson) corresponds to the special representation in which $\lambda_{1}=0, \mu_{1}=2, \lambda_{2}=\lambda$. Only $\lambda_{1}$ is distinct from the other integers and so $2 r+1=1$ in the notation of Lusztig [26, II, top of p. 221], that is $r=0$ and by Lusztig we conclude that this special representation is associated with $2^{r}=1$ representations (i.e. no others). After Lusztig [28] this association is just that which describes the representation occurring in the left cell containing the above special representation which we conclude is irreducible, as required. (Further calculations of this nature will be left to the reader.)

Finally we remark that this is the only not too easy example where we can check our conclusions concerning multiplicities by appealing directly to the 
Kazhdan-Lusztig polynomials and even here I had to ask a student, A. Melnikov, to do the calculation of the Kazhdan-Lusztig polynomials for me (actually the relevant multiplicities were also calculated by Jantzen even before Kazhdan-Lusztig for this case). We must in fact show (for $\alpha=\alpha_{3}$ in type $C_{3}$ ) that $\left[L(-\lambda,-\lambda): V\left(-\lambda,-s_{\alpha} \lambda\right)\right]=1$ where $\lambda=\bar{\omega}_{3}$. Noting that $w_{\lambda}=-1$ it follows from 2.4 (recall that $\lambda$ is antidominant in 2.4 and dominant here) that

$$
\begin{aligned}
{\left[L(-\lambda,-\lambda): V\left(-\lambda,-s_{\alpha} \lambda\right)\right] } & =\left[M(\lambda): L\left(w_{\lambda^{*}}^{0} s_{\alpha} \lambda\right)\right] \\
& =\left[M(\lambda): L\left(w_{\lambda^{*}}^{0} s_{\alpha^{*}} w_{\lambda}^{0} \lambda\right)\right] \\
& =P_{e, w}(1): w=w_{\lambda^{*}}^{0} s_{\alpha^{*}} w_{\lambda}^{0},
\end{aligned}
$$

by the truth of the Kazhdan-Lusztig conjecture and [15, 2.14b]. (Here we need $w$ to be of maximal length in its right $W_{\lambda}^{0}$ coset). Now $w_{\lambda}^{0}=s_{1} s_{2} s_{1}$ and $s_{\alpha}=s_{3}$ (where $s_{i}=s_{\alpha_{i}}$ so we conclude that $w=s_{1} s_{2} s_{3} s_{1} s_{2} s_{1}$ and as required $P_{e, w}(1)=1$. Had we wished to do the same calculation for $B_{3}$ with $\alpha=\alpha_{3}$ then because $\alpha$ is now short we must replace $\alpha$ by its affiliated root $\bar{\alpha}=s_{\alpha_{3}} \alpha_{2}$ and then $w$ becomes $w_{\lambda^{*}}^{0} s_{\bar{\alpha}^{*}} w_{\lambda}^{0}=21323121$. In fact $P_{e, w}(1)=2$, which agrees with the multiplicity of 2 appearing in type $B_{3}$ for $\alpha=\alpha_{3}$.

4.6. The method described in 4.3 allows one to treat the only case in $F_{4}$ for which $\operatorname{dim} V_{B^{\prime \prime}}(\mu)>1$ (and of course the case in $G_{2}$ but which is trivial anyway). These methods fail however to treat two cases in type $E_{8}$ (when $\left.\alpha=\alpha_{3}, \alpha_{4}\right)$ and two families of cases in types $B_{n}, C_{n}, D_{n}$. These cases are all settled by calculating the lengths of the corresponding left cell which always turns out to be $\leq 2$. Under the heading "representation" we give the special representation generated by the Goldie rank polynomial associated to $J_{\max }(\lambda)$ using the notation of Lusztig. We then use the truth of Lusztig's conjecture on left cells settled by Lusztig [28] himself to compute cell length. The calculation is the same as that outlined in 4.5. The result is given in the table under the heading "length". In type $E_{8}$ and one of the families in types $B_{n}, C_{n}, D_{n}$ the cells are irreducible and so $U(\mathfrak{g}) / J_{\max }(\lambda)$ is a principal series module by 3.14 and the required multiplicity is calculated from 3.17(i). In the remaining cases the cells have length 2 and little more work is needed as described below.

4.7. Take $\mathfrak{g}$ of type $D_{n}$. with $\alpha=\alpha_{2 m+1}: 3 m+1 \geq n>2 m+1$. Then $\operatorname{dim} V_{B^{\prime \prime}}(\mu)=2^{3 m+2-n}$. Let $M$ be the corresponding induced module which by 3.4 can be assumed to be simple. Under the heading $\tilde{B}^{\prime \prime \prime}$ we give a subbasis of $R^{+}$(determined as before by inspired guesswork using the description of $B_{0}$ ) such that $B^{\prime \prime \prime}:=w \tilde{B}^{\prime \prime \prime} \subset B$ for some $w \in W$ and $\operatorname{dim} V_{B^{\prime \prime \prime}}(w \lambda)=2^{3 m+1-n}$. (Fortunately we do not have to calculate $w$.) Furthermore card $R^{\prime \prime \prime}=2$ "degree" and so by 3.4 we can assume with loss of generality (in effect with a possible change of $w$ ) that the induced module $M^{\prime}$ is simple and $J_{\max }(\lambda)=\operatorname{Ann} M^{\prime}$. Now we have embeddings $U(\mathfrak{g}) / J_{\max }(\lambda) \hookrightarrow L(M, M)$ and $U(\mathfrak{g}) / J_{\max }(\lambda) \hookrightarrow$ $L\left(M^{\prime}, M^{\prime}\right)$ and by $[16,5.21(2)]$ that $\operatorname{rk} L(M, M)=\operatorname{dim} V_{B^{\prime \prime}}(\mu)=2^{2 m+2-n}$ 
whereas $\operatorname{rk} L\left(M^{\prime}, M^{\prime}\right)=2^{3 m+1-n}$. Since the cell length is 2 it follows from 3.15 that the second embedding must in fact be an isomorphism and then we calculate multiplicity via 3.17(i). The first interesting case occurs when $n=7$, $m=2$, that is in $D_{7}$ for $\alpha=\alpha_{5}$. Then $\operatorname{card} B^{\prime \prime}=5$, yet $\operatorname{dim} V_{B^{\prime \prime}}(\mu)=2$ so the multiplicity may fail to reach its anticipated value of 5 . Curiously one cannot find a subbasis $C$ of $R$ such that $\left(\alpha, \bar{\omega}_{5}\right)=1$ for all $\alpha \in C$ and card $C=5$. This was checked by $\mathrm{D}$. Charcon on the Weizmann Institute computer. (I later realized that this could also be checked by a hand computation.) In our choice of $B^{\prime \prime \prime}$ we have card $B^{\prime \prime \prime}=4$, yet $B^{\prime \prime \prime}$ is of type $A_{2} \times A_{2}$ while $B^{\prime \prime}$ is of type $A_{2} \times A_{1} \times A_{1} \times A_{1}$ so induction can still lead to the same ideal. Finally $\operatorname{dim} V_{B^{\prime \prime \prime}}(\mu)=1$ so our ideal is completely prime. The multiplicity of $E_{\text {ad }}$ in $U(\mathfrak{g}) / J_{\max }(\lambda)$ equals 4 . Deleting the $A_{1}$ factor in $B^{\prime \prime}$ contributing to the factor 2 in $\operatorname{dim} V_{B^{\prime \prime}}(\mu)$ we obtain in an obvious fashion an induced module from a one dimensional representation whose annihilator $J$ satisfies by 3.19 and 3.17 (ii) the relation $\left[U(\mathfrak{g}) / J: E_{\mathrm{ad}}\right] \leq 7-4 \leq 3$. We conclude that $\left[J / J_{\min }(\lambda): E_{\text {ad }}\right] \geq 4$. Yet $d(U(\mathfrak{g}) / J)=\operatorname{card} R-10>d\left(U(\mathfrak{g}) / J_{\max }(\lambda)\right)$ and so $J \varsubsetneqq J_{\max }(\lambda)$. Since $\left[J_{\max }(\lambda) / J_{\min }(\lambda): E_{\text {ad }}\right]=4$ we conclude that $\left[J_{\max }(\lambda) / J: E_{\mathrm{ad}}\right]=0$. Since $J_{\max }(\lambda)$ is a completely prime induced ideal which obviously cannot be generated by a copy of $E_{\text {ad }}$ we really do obtain a third way in which Gupta's theorem can fail for special $\lambda$. The reader may amuse himself by finding similar failures in types $D_{3 k+7}: k=1,2,3, \ldots$.

4.8. A similar analysis applies in type $C_{n}$ with $\alpha=\alpha_{2 m}: 3 m>n>2 m$. We may also distinguish generalized principal series modules by their Goldie ranks. The relevant data is given in the table. One finds that $\operatorname{rk}\left(U(\mathfrak{g}) / J_{\max }(\lambda)\right)=$ $2^{3 m-n-1}$ and so is completely prime for $3 m=n+1$. Yet the multiplicity equals $2 n-4 m+1$ which coincides with card $B^{\prime \prime}=n-m$ in the above case so we do not immediately obtain a counterexample to Gupta's theorem for $\lambda$ special.

4.9. Finally consider the case when $\mathfrak{g}$ is of type $B_{n}$ with $\alpha=\alpha_{2 m}: 3 m>$ $n>2 m$. Here curiously both principal series modules have the same Goldie rank (but this does not imply equality with $U(\mathfrak{g}) / J_{\max }(\lambda)$ because the Goldie skew-fields may differ). In this we use the estimate in 3.18 to distinguish the principal series modules, with $B_{1}=B^{\prime \prime}, B_{2}=B^{\prime \prime \prime}$, as tabulated.

The necessary data is given in the table. Alternatively one may use the $C_{n}$ computation for $\alpha_{2 m}$ noting that $B^{\prime \prime \prime}$ has exacty one long root $\beta$ such that $\left(\beta^{\vee}, \mu\right)=1$. Via 3.17 the multiplicity $2 n-4 m$ is exactly one less than the corresponding value for $C_{n}$. Again we do not get immediate counterexamples to Gupta's theorem for $\lambda$ special.

4.10. There is a fourth way we can get Gupta's theorem to fail for $\lambda$ special, namely we find distinct nonmaximal ideals $J_{1}, J_{2}$ such that $\left[J_{1} / J_{\min }(\lambda): E_{\text {ad }}\right]=$ $\left[J_{2} / J_{\text {min }}(\lambda): E_{\text {ad }}\right]=\left[J_{\max }(\lambda) / J_{\min }(\lambda): E_{\text {ad }}\right]$ which are induced and completely prime. Obviously both $J_{1} / J_{\min }(\lambda)$ and $J_{2} / J_{\min }(\lambda)$ cannot be generated by a 
copy of the adjoint representation since this would imply that they are equal. The phenomenon can be relatively easily exhibited if $\left[J_{\max }(\lambda) / J_{\min }(\lambda): E_{\text {ad }}\right]$ is too small. We illustrate this in type $E_{8}$ with $\alpha=\alpha_{4}$. Here the above value is 6 rather than card $B^{\prime \prime}=7$. The factor 2 in the Goldie rank comes from $\alpha_{1} \in B^{\prime \prime}$. Take $J_{1}$ to be the ideal obtained by deleting $\alpha_{1}$ (see 4.7). As in 4.7 we conclude that $J_{1}$ is completely prime, that $(3.17(1))\left[U(\mathfrak{g}) / J_{1}\right.$ : $\left.E_{\text {ad }}\right] \leq 8-6$ so $\left[J_{1} / J_{\min }(\lambda): E_{\text {ad }}\right]=6$ and $d\left(U(\mathfrak{g}) / J_{1}\right)=\operatorname{card} R-42$ (whereas $\left.d\left(U(\mathfrak{g}) / J_{\max }(\lambda)\right)=\operatorname{card} R-44\right)$. Now from $\tilde{B}^{\prime \prime}$ (given under the heading invariant parabolic) delete $\beta_{5}:=\left(00_{0}^{1} 1111\right)$-which is eventually carried into $\alpha_{5}$ and apply $s_{4}$ to the remaining roots. One checks that $\left(\gamma^{\vee}, \lambda\right)=1$ for all $\gamma \in$ $s_{4}\left(\tilde{B}^{\prime \prime}-\{\beta\}\right)=: \tilde{B}^{\prime \prime \prime}$ and that $\tilde{B}^{\prime \prime \prime}$ is conjugate to $B^{\prime \prime \prime}:=\left\{\alpha_{1}, \alpha_{2}, \alpha_{4}, \alpha_{6}, \alpha_{7}, \alpha_{8}\right\}$ which is of type $A_{1} \times A_{2} \times A_{3}$. Thus, as above, the corresponding induced ideal $J_{2}$ is completely prime, satisfies $\left[J_{2} / J_{\text {min }}(\lambda): E_{\text {ad }}\right]=6$ and $d\left(U(\mathfrak{g}) / J_{2}\right)=$ card $R-20$, so is distinct from $J_{1}$.

\section{THE MULTIPLICITY FORMULA}

Take $-\lambda \in \mathfrak{h}^{*}$ dominant.

5.1. We now describe how to calculate $\left[U(\mathfrak{g}) / J_{\max }(\lambda): E\right]$ for each $\lambda \in \mathfrak{h}^{*}$. As in [23, 4.3] we set $B_{\lambda}^{1}=\left\{\alpha \in B_{\lambda} \mid\left(\alpha^{\vee}, \lambda\right)=-1\right\}$. By [23, 3.5] we can assume that $B_{\lambda}^{1} \subset B$ without loss of generality.

Lemma. For each $-\lambda \in \mathfrak{h}^{*}$ dominant one has

$$
\left[U(\mathfrak{g}) / J_{\max }(\lambda): E_{\mathrm{ad}}\right]=\operatorname{rank} \mathfrak{g}-\sum_{\alpha \in B_{\lambda}^{1}}\left[L(-\lambda,-\lambda): V\left(-\lambda,-s_{\bar{\alpha}} \lambda\right)\right] .
$$

Recall that $U(\mathfrak{g}) / J_{\min }(\lambda) \cong L(-\lambda,-\lambda)$, that $U(\mathfrak{g}) / J_{\max }(\lambda) \cong V(-\lambda,-\lambda)$ and that the latter module occurs with exactly multiplicity one in $L(-\lambda,-\lambda)$. As noted in $[23,4.3]$ the adjoint representation occurs only in $V(-\lambda,-\lambda)$ and the $V\left(-\lambda,-s_{\bar{\alpha}} \lambda\right): \alpha \in B_{\lambda}^{1}$ which are pairwise nonisomorphic. Furthermore it occurs with exactly multiplicity one in each $V\left(-\lambda,-s_{\bar{\alpha}} \lambda\right): \alpha \in B_{\lambda}^{1}$. Since $\left[L(-\lambda,-\lambda): E_{\mathrm{ad}}\right]=E_{\mathrm{ad}}^{\mathfrak{h}}=$ rank $\mathfrak{g}$, the lemma follows.

5.2. Fix $\alpha \in B_{\lambda}^{1}$ and define $B_{1}$ as in 2.5 . By 2.5 we may replace $[L(-\lambda,-\lambda)$ : $\left.V\left(-\lambda,-s_{\bar{\alpha}} \lambda\right)\right]$ in 5.1 by $\left[L(-\lambda,-\lambda): V\left(-\lambda,-s_{\bar{\alpha}} \lambda\right)\right]_{B_{1}}$. In computing the latter we may assume without loss of generality that $B$ is replaced by $B_{1}$ and that $\lambda=$ $-\bar{\omega}_{\alpha}$. Then we have $V\left(-\lambda,-s_{\bar{\alpha}} \lambda\right) \cong V\left(-w_{B} \lambda,-w_{B} s_{\bar{\alpha}} \lambda\right)=V\left(-\mu,-s_{-w_{B} \bar{\alpha}} \mu\right)$ where $\mu=w_{B} \lambda=\bar{\omega}_{-w_{B} \alpha}$. Since $w_{B} \bar{\alpha}=\overline{w_{B} \alpha}$ (actually $\alpha=\bar{\alpha}$ unless $w_{B}=$ -1 ) the result is the same as if we took $\lambda=\bar{\omega}_{w_{B} \alpha}$, that is on the $-w_{B} \alpha$ corner. Actually it is clear that $w_{B} B_{\lambda}^{1}=-B_{\lambda}^{1}$ and that $\left[L(-\lambda,-\lambda): V\left(-\lambda,-s_{\bar{\alpha}} \lambda\right)\right]$ $=\left[L(-\lambda,-\lambda): V\left(-\lambda,-s_{-\overline{w_{B} \alpha}} \lambda\right)\right]$ so changing from antidominant to dominant $\lambda$ involves no complications. Finally the multiplicities $[L(-\lambda,-\lambda)$ : $\left.V\left(-\lambda,-s_{\bar{\alpha}} \lambda\right)\right]_{B_{1}}$ are given in the table. For the reader's convenience we have presented these results in the form of numbered Dynkin diagrams in which to 
the vertex defining say the root $\alpha$ we have assigned the corresponding multiplicity when $\lambda$ lies on the $\alpha$-corner. It should be noted that the results for $B_{n}$ and $C_{n}$ differ because $\alpha \in B_{\lambda}^{1}$ has to be replaced by its affiliated root $\bar{\alpha}$.

5.3. To illustrate our method we take $\mathfrak{g}$ of type $D_{8}$ with $\lambda=-\left(\bar{\omega}_{3}+\bar{\omega}_{6}\right)$ (notation Bourbaki). For $\alpha=\alpha_{3}$, we have $B_{1}=\left\{\alpha_{1}, \alpha_{2}, \alpha_{3}, \alpha_{4}, \alpha_{5}\right\}$ which is type $A_{5}$. From the table we deduce a multiplicity of 3 in this case. For $\alpha=\alpha_{6}$, we have $B_{1}=\left\{\alpha_{4}, \alpha_{5}, \alpha_{6}, \alpha_{7}, \alpha_{8}\right\}$ which is of type $D_{5}$ and from the table we deduce a multiplicity of 4 . Hence $\left[U(\mathfrak{g}) / J_{\max }(\lambda): E_{\mathrm{ad}}\right]=8-3-4=1$. Again take $\mathfrak{g}$ of type $A_{n}$ with $n=l+k(l+1)$ and

$$
\lambda=-\sum_{i=1}^{l} \bar{\omega}_{(k+1) i} .
$$

Take $\alpha=\alpha_{(k+1) i}$. Then $B_{1}$ is a system of type $A_{2 k+1}$ with $\alpha$ its central root. Each such term gives a contribution of $(k+1)$. Hence $\left[U(\mathfrak{g}) / J_{\max }(\lambda): E_{\mathrm{ad}}\right]=$ $k(l+1)+l-(k+1) l=k$. These examples show that $\left[U(\mathfrak{g}) / J_{\max }(\lambda): E_{\mathrm{ad}}\right]$ can be quite small for $\lambda$ of intermediate singularity.

5.4. In virtue of 3.4 we can essentially ignore the question of whether our induced modules are in fact irreducible. Actually we are implicity getting close to irreducibility by forcing $\operatorname{dim} V_{B^{\prime \prime}}(\mu)$ to be as small as possible. For example when $\operatorname{dim} V_{B^{\prime \prime}}(\mu)=1$ then the annihilator of the induced module is completely prime, hence primitive. Actually we checked in all cases (except $\alpha=\alpha_{2}$ in $F_{4}$ ) that our choice of invariant parabolic led to an irreducible induced module. Here we used a remarkably efficient sufficiency condition for irreducibility due to Jantzen [14, Corollary 3]. (In type $F_{4}$ with $\alpha=\alpha_{2}$ we only checked necessity via [14, Corollary 2].) This can be expressed as follows.

Theorem. Take $B^{\prime \prime} \subset B$ and set $R^{\prime \prime+}=R^{+} \cap \mathbf{N} B^{\prime \prime}$. Choose $\mu \in \mathfrak{h}^{*}$ such that $\left(\mu, \alpha^{\vee}\right) \in \mathbf{N}^{+}$for all $\alpha \in B^{\prime \prime}$. Suppose that for each $\beta \in R^{+}-R^{\prime \prime}$ satisfying $\left(\beta^{\vee}, \mu\right) \in \mathbf{N}^{+}$there exists $\alpha \in R_{\mu}^{0}$ such that $s_{\beta} \alpha \in R^{\prime \prime+}$. Then $M_{B^{\prime \prime}}(\mu):=U(\mathfrak{g}) \otimes_{U\left(\mathfrak{p}_{B^{\prime \prime}}\right)} V_{B^{\prime \prime}}(\mu)$ is irreducible.

As it stands this criterion is a little messy to verify in our situation. However suppose we ignore the hypothesis that $\left(\beta^{\vee}, \mu\right) \in \mathbf{N}^{+}$(that is we check for each $\beta \in R^{+}-\left(R^{\prime \prime+} \cup\left(R_{\mu}^{0} \cap R^{+}\right)\right)$that there exists $\alpha \in R_{\mu}^{0}$ such that $\left.s_{\beta} \alpha \in R^{\prime \prime+}\right)$. Then we can check this criterion on the conjugate pair $\tilde{B}^{\prime \prime}=w B^{\prime \prime}, \lambda=w \mu$. Indeed if we set $\tilde{R}^{\prime \prime}=\mathbf{Z} \tilde{B}^{\prime \prime} \cap R$ it is equivalent to requiring that for each $\beta \in R-\left(\tilde{R}^{\prime \prime} \cup R_{\lambda}^{0}\right)$ that there exists $\alpha \in R_{\lambda}^{0}$ such that $s_{\beta} \alpha \in \tilde{R}^{\prime \prime}$. Since $\lambda$ is given to us (whereas $\mu$ has to be computed) this is much easier. Except in $F_{4}$ this was enough to give irreducibility for the tabulated values of $\tilde{B}^{\prime \prime}$.

5.5. In the above we are in some sense checking more than we need and it is relevant to ask if this can give additional information. In fact we have with 
$B^{\prime \prime}, \mu$ as in 5.4 the

Corollary. Suppose that for each $\beta \in R-\left(R^{\prime \prime} \cup R_{\mu}^{0}\right)$ there exists $\alpha \in R_{\mu}^{0}$ such that $s_{\beta} \alpha \in R^{\prime \prime}$. Then $L\left(M_{B^{\prime \prime}}(\mu), M_{B^{\prime \prime}}(\mu)\right)$ is semisimple. In particular Ann $M_{B^{\prime}}(\mu)$ is a maximal ideal.

By 5.4 both $M_{B^{\prime \prime}}(\mu)$ and $M_{B^{\prime \prime}}\left(-w_{B^{\prime \prime}} \mu\right)$ are irreducible. Set

$$
S=L\left(L_{B^{\prime \prime}}(\mu), M_{B^{\prime \prime}}(\mu)\right), \quad S^{\prime}=L\left(M_{B^{\prime \prime}}\left(-w_{B^{\prime \prime}} \mu\right), M_{B^{\prime \prime}}\left(-w_{B^{\prime \prime}} \mu\right)\right) .
$$

Since $M_{B^{\prime \prime}}(\mu)$ is simple in $\mathfrak{O}$ it is self-dual and so $S$ is isomorphic to the generalized principal series module $L_{B^{\prime \prime}}(-\mu,-\mu):=L\left(\left(M_{B^{\prime \prime}}(\mu) \otimes M_{B^{\prime \prime}}(\mu)\right)^{*}\right)$. Similarly $S^{\prime}$ is isomorphic to $L_{B^{\prime \prime}}\left(w_{B^{\prime \prime}} \mu, w_{B^{\prime \prime}} \mu\right)$. Yet as noted in [9, 2.10] suitable integration gives a nondegenerate $U(\mathfrak{g}) \otimes U(\mathfrak{g})$ invariant pairing between $L_{B^{\prime \prime}}(-\mu,-\mu)$ and $L_{B^{\prime \prime}}\left(w_{B^{\prime \prime}} \mu, w_{B^{\prime \prime}} \mu\right)$. (One requires that $V_{B^{\prime \prime}}(\mu) \otimes V_{B^{\prime \prime}}(\mu)$ should be contragredient as an $\mathfrak{r}_{B^{\prime \prime}} \times \mathfrak{r}_{B^{\prime \prime}}$ module to $V_{B^{\prime \prime}}\left(-w_{B^{\prime \prime}} \mu\right) \otimes V_{B^{\prime \prime}}\left(-w_{B^{\prime \prime}} \mu\right)$. A purely algebraic description of this pairing for the case $B^{\prime \prime}=\varnothing$ can be found in $[10,9.6 .9 ; 11, \S 6]$.)

We conclude that $S, S^{\prime}$ are nondegenerately paired. On the other hand by [18, III, 4.13] $d(S / \operatorname{Soc} S)<d(S)$ with a similar assertion for $S^{\prime}$. Hence $S=\operatorname{Soc} S$ which is hence semisimple. Since $U(\mathfrak{g}) / \operatorname{Ann} M_{B^{\prime \prime}}(\mu)$ is a submodule of $S$ it is also semisimple and because $\operatorname{Ann} M_{B^{\prime \prime}}(\mu)$ is primitive hence prime this quotient must be simple.

Remark. Given that Ann $M_{B^{\prime \prime}}(\mu)$ is maximal, semisimplicity is already a consequence of the dimension estimate $d(S / \operatorname{Soc} S)$ (cf. 3.15). Of course the reader might guess that we were really trying to prove simplicity under these hypotheses. Unfortunately this is false in type $D_{7}$ with $\lambda=\bar{\omega}_{5}, \tilde{B}^{\prime \prime}$ as in the table and $\mu=w \lambda, B^{\prime \prime}:=w \tilde{B}^{\prime \prime} \subset B$. In fact the calculation in 4.7 shows the generalized principal series module has length 2 whilst the reader can amuse himself by checking that the hypothesis of 5.5 holds for these choices.

5.6. Recall that $\lambda \in \mathfrak{h}^{*}$ is assumed antidominant and that we can take $B_{\lambda}^{1} \subset B$. Gupta [12] calls $\lambda$ nonspecial if $\left(\lambda, \gamma^{\vee}\right)=-1, \gamma \in R_{\lambda}$ (equivalently in $R$ ) implies $\gamma \in B_{\lambda}$ (hence in $B$ ). In $[23,4.2]$ we defined $\lambda$ to be semiregular if $\left[L(-\lambda,-\lambda): V\left(-\lambda,-s_{\bar{\alpha}} \lambda\right)\right]=1$ for all $\gamma \in B_{\lambda}^{1}$. Gupta established [12] that for $\lambda$ nonspecial an induced ideal $I \supsetneqq J_{\min }(\lambda)$ is generated over $J_{\min }(\lambda)$ by one copy of the adjoint representation occurring in $I / J_{\min }(\lambda)$. (Here induction is assumed to be from a $U(\mathfrak{p})$ module of dimension one.) In [23, 4.4] we established (a slight modification of) this result and its converse for $\lambda$ semiregular.

Now assume $\lambda$ nonspecial and take $\alpha \in B_{\lambda}^{1} \subset B$. Given $\beta \in B_{\lambda}^{0} \subset B$ we must have $(\beta, \alpha)=0$ for otherwise $\gamma:=s_{\beta} \alpha$ satisfies $\left(\lambda, \gamma^{\vee}\right)=-1$ and $\gamma \in R_{\lambda}-B_{\lambda}$. We conclude that the connected component of $\{\alpha\} \cup B_{\lambda}^{0}$ containing $\alpha$ is just $\{\alpha\}$ (which is a system of type $A_{1}$ ). Thus $\alpha=\bar{\alpha}$ and from our table that $\left[L(-\lambda,-\lambda), V\left(-\lambda,-s_{\bar{\alpha}} \lambda\right)\right]=1$. We conclude that $\lambda$ nonspecial implies 
$\lambda$ semiregular. On the other hand our table gives a myriad of situations when these multiplicities are one and yet $\lambda$ is special.

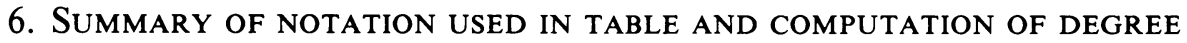

6.1. We recall that for each simple Lie algebra designated by "type" (in the table) we select $\alpha \in B$ designated by "root" and compute the "multiplicity" of $V\left(\lambda, s_{\bar{\alpha}} \lambda\right)$ in $U(\mathfrak{g}) / J_{\max }(\lambda)$ when $\lambda$ lies on the $\alpha$ corner. To do this we set $B^{\prime}=B-\{\alpha\}$ and compute the unique $H \in \mathfrak{h}$ forming an s-triple $X, H, Y$ with $X=\sum_{\beta \in B^{\prime}} X_{\beta}, Y \in \mathfrak{r}_{B^{\prime}}$. Then $\left[H, X_{\alpha}\right]$ is proportional to $X_{\alpha}$ with the proportionality factor being designated by "coefficient". We compute the basis $B_{0} \subset R^{+}$for those roots $\beta \in R$ satisfying $\left[H, X_{\beta}\right]=0$. We extend this to the subbasis $B_{0} \amalg B_{1}$ of $R$ having the property that each $\beta \in R$ satisfying $\left[H, X_{\beta}\right]=X_{\beta}$ takes the form $\beta=\gamma+\delta, \gamma \in B_{1}, \delta \in \mathbf{N} B_{0} \cap R^{+}$. In the table the sets $B_{0}, B_{1}$ are described using the conventions and notations of Bourbaki [7]. We use this data to compute the nilpotent orbit $G X$ designated by "orbit (1)" in the table. This is described for the exceptional algebras by Dynkin data and for the classical algebras using the notation in Carter [8, 13.1].

6.2. In the table "orbit (2)" designates the unique dense nilpotent orbit in the zero variety $\mathscr{V}\left(J_{\max }(\lambda)\right)$ of $\operatorname{gr} J_{\max }(\lambda)$ when $\lambda$ lies on the $\alpha$-corner. After 3.7 one has $G X \subset \mathscr{V}\left(J_{\max }(\lambda)\right)$, so $G X$ is the required orbit if and only if dimensions coincide. Let $\tau\left(B^{\prime}\right)$ denote the Macdonald representation (6.5) of $W$ generated by the polynomial formed from the product of roots in $R^{\prime+}:=$ $\mathbf{N} B^{\prime} \cap R^{+}$and let $l_{\tau\left(B^{\prime}\right) \otimes s n}$ denote the lowest "degree" in which $\tau\left(B^{\prime}\right) \otimes s n$ occurs in $S(\mathfrak{h})$. After 3.11 one has $\operatorname{dim} \mathscr{V}\left(J_{\max }(\lambda)\right) \geq \operatorname{card} R-2 l_{\tau\left(B^{\prime}\right) \otimes s n}$. From our knowledge of $B_{0}$ and $B_{1}$ we guess a subbasis $\tilde{B}^{\prime \prime} \subset R^{+}$such that $\left(\bar{\omega}_{\alpha}, \beta\right)>0$ for all $\beta \in \tilde{B}^{\prime \prime}$ and card $\mathbf{N} \tilde{B}^{\prime \prime} \cap R^{+}=l_{\tau\left(B^{\prime}\right) \otimes s n}$. Let $w \in W$ satisfy $B^{\prime \prime}:=w \tilde{B} \subset B$ (fortunately $w$ never has to be computed and of course $B^{\prime \prime}$ is not uniquely determined) and set $\mu=w \lambda$. In the table "subtype" designates the root system defined by $B^{\prime \prime}$. By construction there exists a finite dimensional simple $U\left(\mathfrak{p}_{B^{\prime \prime}}\right)$ module $V_{B^{\prime \prime}}(\mu)$ with highest weight $\mu-\rho$. Its dimension which unfortunately is not always one is given in the table. The annihilator $J$ of the induced module $M_{B^{\prime \prime}}(\mu):=U(\mathfrak{g}) \otimes_{U\left(\mathfrak{p}_{B^{\prime \prime}}\right)} V_{B^{\prime \prime}}(\mu)$ satisfies $d(U(\mathfrak{g}) / J)=$ card $R-\operatorname{card}\left(\mathbf{Z} B^{\prime \prime} \cap R\right)$ from which we conclude that $\sqrt{J}=J_{\max }(\lambda)$ and that "orbit (2)" is the Richardson orbit whose closure in $G \mathfrak{m}_{B}$. Furthermore we show that $\mathfrak{p}_{B^{\prime \prime}}$ is (for our choices) an invariant parabolic subalgebra and so when $\operatorname{dim} V_{B^{\prime \prime}}(\mu)=1$ we conclude that the required multiplicity is just card $B^{\prime \prime}$.

6.3. When $\operatorname{dim} V_{B^{\prime \prime}}(\mu)>1$ some additional information is computed, not all of which is strictly necessary. Using $[8,13.1]$ we compute the cardinality of the component group $A_{x}$ of "orbit (2)". It is always $\leq 4$ thus indicating that not too much difficulty will be encountered (cf. 3.21, 3.23). In the table we designate by "representation" the Goldie rank representation associated to $J_{\max }(\lambda)$ using the notation in [26, I, §8] for the classical algebras and in [3, §4] 
for $E_{8}$. (This representation is just the Macdonald representation associated to $B^{\prime \prime}$.) In the table "length" designates the length of a left cell containing the representation. This is computed by the truth of Lusztig's conjecture [26, II, $\S 10$ ] as demonstrated by Lusztig himself [28]. When this length is strictly greater than one we determine a new subbasis $\tilde{B}^{\prime \prime \prime}$ satisfying the same conditions as $\tilde{B}^{\prime \prime}$. Let $w^{\prime} \in W$ satisfy $B^{\prime \prime \prime}:=w^{\prime} \tilde{B}^{\prime \prime \prime}$ and set $\mu^{\prime}=w^{\prime} \lambda$. Induction from the simple $U\left(\mathfrak{p}_{B^{\prime \prime \prime}}\right)$ module $V_{B^{\prime \prime \prime}}\left(\mu^{\prime}\right)$ gives an alternative description of $J_{\max }(\lambda)$ and comparison enables us to compute the required multiplicity.

Take $\lambda$ on a corner. Then $U(\mathfrak{g}) / J_{\max }(\lambda)$ is always a principal series module. This allows one to calculate multiplicities of other $\mathfrak{k}$ types (for corner weights) and scalar factors in certain Goldie rank polynomials. For all this it is enough to take $\lambda=\bar{\omega}_{\alpha}: \alpha \in B$. When $\operatorname{dim} V_{B^{\prime \prime}}(\mu)=1$ our assertion follows from 3.20, 3.21 whose $\mathfrak{p}_{B^{\prime \prime}}$ is the invariant parabolic in question and of course $\operatorname{rk} U(\mathfrak{g}) / J_{\max }\left(\bar{\omega}_{\alpha}\right)=1$. When $\operatorname{dim} V_{B^{\prime \prime}}(\mu)>1$ additional computation is needed. In all cases except $\alpha=\alpha_{2}$ in type $F_{4}$ an analysis along the lines of 4.6, 4.7 suffices. One has $\operatorname{rk}\left(U(\mathfrak{g}) / J_{\max }\left(\bar{\omega}_{\alpha}\right)\right)=\operatorname{dim} V_{B^{\prime \prime \prime}}\left(\mu^{\prime}\right)$ when the latter is listed. When in addition $\operatorname{dim} V_{B^{\prime \prime \prime}}\left(\mu^{\prime}\right)<\operatorname{dim} V_{B^{\prime \prime}}(\mu)$ this primitive quotient is described as a principal series module by $B^{\prime \prime \prime}$ and otherwise by $B^{\prime \prime}$. (For example in type $B_{n}$ with $\alpha=\alpha_{m}: 3 m>n>2 n$, it is $B^{\prime \prime}$ which describes $U(\mathfrak{g}) / J_{\max }\left(\bar{\omega}_{\alpha}\right)$ as a (generalized) principal series module.) For $\alpha=\alpha_{2}$ in type $F_{4}$ the left cell lengths associated to the special representation $\mathscr{X}_{12,1}$ range between 5 and 7 so our previous analysis fails. Here 3.24 and 3.25 are used.

6.4. We have not yet explained how to compute "degree" for the classical Lie algebras. Here we present the appropriate formulae which were deduced from $[25, \S 2]$.

Let $0 \leq \alpha_{1} \leq \alpha_{2} \leq \cdots \leq \alpha_{k}$ be a partition of $n$ denoted in short by $\alpha$. Set

$$
|\alpha|=\sum_{i=1}^{k} \alpha_{i}, \quad m(\alpha)=\sum_{i=1}^{k}\left(\begin{array}{c}
\alpha_{i} \\
2
\end{array}\right) .
$$

If $\alpha^{*}$ is the dual partition one easily checks that

$$
|\alpha|=\left|\alpha^{*}\right|, \quad m\left(\alpha^{*}\right)=\sum_{i=1}^{k}(k-i) \alpha_{k-i+1} .
$$

Now suppose $\mathfrak{g}$ is simple of type $A_{n-1}$. Then the Weyl group is isomorphic to the symmetric group $S_{n}$ on $n$ elements and $\hat{S}_{n}$ is parametrized by the partitions of $n$.

If $\alpha$ is a partition, let $W^{\prime}$ be a subgroup of $W$ isomorphic to $S_{\alpha_{1}^{*}} \times S_{\alpha_{2}^{*}} \times$ $\cdots \times S_{\alpha_{i}^{*}}$ generated by a subbasis $B^{\prime} \subset B$ of type $A_{\alpha_{1}^{*}-1} \times A_{\alpha_{2}^{*}-1} \times \cdots \times A_{\alpha_{i}^{*}-1}$. Macdonald's construction [30] associates to the sign representation of $W^{\prime}$ an irreducible representation $\tau\left(B^{\prime}\right)$ or $E_{\alpha}$ of $W$ satisfying

$$
l_{\tau\left(B^{\prime}\right)}=\operatorname{card}\left(\mathbf{N} B^{\prime} \cap R^{+}\right)=m\left(\alpha^{*}\right) .
$$


Since tensoring by sign corresponding to taking dual partitions we conclude that

$$
l_{\tau\left(B^{\prime}\right) \otimes s n}=m(\alpha) \text {. }
$$

Let $\mathfrak{g}$ be simple of type $B_{n}$ (resp. $C_{n}$ ). Let $\alpha=\left(\alpha_{1} \leq \alpha_{2} \leq \cdots \leq \alpha_{k+1}\right)$, $\beta=\left(\beta_{1} \leq \beta_{2} \leq \cdots \leq \beta_{k}\right)$ be partitions such that

$$
\sum_{i=1}^{k+1} \alpha_{i}+\sum_{j=1}^{k} \beta_{j}=n, \quad \alpha_{i} \geq 0, \beta_{j} \geq 0 .
$$

In the dual partitions $\alpha^{*}, \beta^{*}$ we assume all terms $>0$ and take $D_{1}$ to be the empty root system. Let $R^{\prime} \subset R$ be a root system of type

$$
\begin{gathered}
D_{\alpha_{1}^{*}} \times D_{\alpha_{2}^{*}} \times \cdots \times D_{\alpha_{m^{*}}^{*}} \times B_{\beta_{1}^{*}} \times \cdots \times B_{\beta_{m^{\prime \prime}}^{*}} \\
\text { (resp. } \left.D_{\alpha_{1}^{*}} \times D_{\alpha_{2}^{*}} \times \cdots \times D_{\alpha_{m^{*}}^{*}} \times C_{\beta_{1}^{*}} \times \cdots \times C_{\beta_{m^{\prime \prime}}^{*}}\right) .
\end{gathered}
$$

Then Macdonald's construction associates to the sign representation of $W^{\prime}$ an irreducible representation $\tau\left(R^{\prime}\right)$ or $E_{\alpha, \beta}$ of $W$ satisfying

$$
l_{\tau\left(R^{\prime}\right)}=\operatorname{card}\left(R^{\prime} \cap R^{+}\right)=2 m\left(\alpha^{*}\right)+2 m\left(\beta^{*}\right)+\left|\beta^{*}\right| .
$$

Tensoring by sign is no longer simply taking dual partitions; but one checks from $[25,2.4]$ using $3.10(*)$ and the formula in $[3, \S 2]\left(\right.$ see $\left.P_{\rho}(q)\right)$ that

$$
l_{\tau\left(R^{\prime}\right) \otimes s n}=2 m(\alpha)+2 m(\beta)+|\alpha| .
$$

Whilst it is true (though not obvious) that the above construction gives a parametrization of $\hat{W}$ in types $B_{n}$ and $C_{n}$ this does not mean (unlike type $A_{n}$ ) that all Macdonald representations have such a presentation. In particular we can have a subset $B^{\prime \prime} \subset B$ not of the above type and then we have to be able to describe the Macdonald representation $\tau\left(B^{\prime \prime}\right)$ in terms of the above parametrization. This question is considered in 6.5.

Let $\mathfrak{g}$ be simple of type $D_{n}$. Let $\alpha=\left(\alpha_{1} \leq \cdots \leq \alpha_{k}\right), \beta=\left(\beta_{1} \leq \beta_{2} \leq \cdots \leq\right.$ $\beta_{k}$ ) be partitions such that

$$
\sum_{i=1}^{k} \alpha_{i}+\sum_{j=1}^{k} \beta_{j}=n, \quad \alpha_{i} \geq 0, \beta_{j} \geq 0 .
$$

Set $\alpha^{\prime}=\left(0 \leq \alpha_{1} \leq \cdots \leq \alpha_{k}\right)$. Let $E_{\alpha, \beta}$ be the representation of $W$ obtained by restriction of the representation $E_{\left(x^{\prime}, \beta\right.}$ of the Weyl group of type $B_{n}$ containing $W$ as a subgroup of index 2 . Then $E_{\alpha, \beta} \cong E_{\beta, \alpha}$ is irreducible if $\alpha \neq \beta$ while $E_{\alpha, \alpha \gamma}$ splits into two distinct irreducible $W$ modules $E_{\alpha, \alpha}^{\mathrm{I}}, E_{\alpha, \alpha}^{\mathrm{II}}$. From [25, 2.7(iii)] we obtain

$$
\begin{aligned}
& l_{E_{n, \beta}}=2 m\left(\alpha^{*}\right)+2 m\left(\beta^{*}\right)+\inf \left(\left|\alpha^{*}\right|,\left|\beta^{*}\right|\right), \\
& l_{E_{n, \beta} \otimes s n}=2 m(\alpha)+2 m(\beta)+\inf (|\alpha|,|\beta|),
\end{aligned}
$$

where we can take either $E_{\alpha, \beta}=E_{\alpha, \beta}^{\mathrm{I}}$ or $E_{\alpha, \beta}=E_{\alpha, \beta}^{\mathrm{II}}$ when $\alpha=\beta$. 
6.5. Take $\tau \in \hat{W}$. By definition $l_{\tau}$ is just the lowest degree in which $\tau$ occurs in $S(\mathfrak{h})$. Following [18, II, 1.1] we call $\tau$ univalent if it occurs with multiplicity one in that degree and let $M_{\tau}$ denote the associated univalent module. Let $R^{\prime} \subset R$ be a root subsystem with Weyl group $W^{\prime}$ and set $\mathfrak{h}^{\prime}=\left.\mathfrak{h}\right|_{R^{\prime \perp}}$. In [29, 3.2] Lusztig and Spaltenstein point out that Macdonald's construction extends to a truncated induction functor $j_{W^{\prime}}^{W}$ carrying a univalent representation $\tau^{\prime}$ of $W^{\prime}$ to a univalent representation $\tau$ of $W$. One has $M_{\tau}=\mathrm{C} W M_{\tau^{\prime}}$ and so $M_{\tau}$ identifies with the irreducible subrepresentation $\tau$ of $\operatorname{Ind}_{W^{\prime}}^{W} \tau$ satisfying $l_{\tau}=l_{\tau^{\prime}}$. We remark that the sign representation $s n$ of $W$ is univalent with $l_{s n}=$ card $R^{+}$and that we recover Macdonald's construction by taking $\tau^{\prime}$ to be the sign representation of $W^{\prime}$.

Let $R^{\prime}, R^{\prime \prime}$ be root subsystems of $R$ with Weyl groups $W^{\prime}, W^{\prime \prime}$ respectively. If $R^{\prime}, R^{\prime \prime}$ are of the same type, then card $R^{\prime}=\operatorname{card} R^{\prime \prime}$ and $W^{\prime} \cong W^{\prime \prime}$ and so we conclude that

$$
j_{W^{\prime}}^{W}(s n) \cong j_{W^{\prime \prime}}^{W}(s n) .
$$

Yet (outside type $A_{n}$ ) it can happen that (*) holds even if $R^{\prime}, R^{\prime \prime}$ are of different type. This fact was already reported by Macdonald [30, §3]. To analysis this (for the situations of concern to us) let $R_{1}, R_{2}$ be mutually orthogonal root subsystems of $R$ and $R^{\prime}, R^{\prime \prime}$ root subsystems of $R_{1}$. Let $W_{1}, W_{2}, W^{\prime}, W^{\prime \prime}$ denote the corresponding Weyl subgroups of $W$ with $s n$ denoting the sign representation of the group in question.

Lemma. If $j_{W^{\prime}}^{W_{1}}(s n) \cong j_{W^{\prime \prime}}^{W_{1}}(s n)$ then $j_{W^{\prime} \times W_{2}}^{W}(s n) \cong j_{W^{\prime \prime} \times W_{2}}^{W}(s n)$.

One easily checks that induction by stages applies to $j$. Hence

$$
j_{W^{\prime} \times W_{2}}^{W}(s n)=j_{W_{1} \times W_{2}}^{W}\left(j_{W^{\prime} \times W_{2}}^{W_{1} \times W_{2}}(s n)\right)=j_{W_{1} \times W_{2}}^{W}\left(j_{W^{\prime}}^{W_{1}}(s n) \otimes s n\right),
$$

from which the required result follows.

6.6. Let $n$ be a positive integer $\geq 2$. For each $i \in\{1,2, \ldots, n\}$ define an involution $\sigma_{i}$ on $\mathbf{C}\left[\varepsilon_{1}, \varepsilon_{2}, \ldots, \varepsilon_{n}\right]$ through $\sigma_{i} \varepsilon_{j}=\varepsilon_{j}$ if $i \neq j$ and $\sigma_{i} \varepsilon_{i}=-\varepsilon_{i}$. Set

$$
S=\left(1+\sigma_{1}\right)\left(1+\sigma_{2}\right), \quad A=\left(1-\sigma_{1}\right)\left(1-\sigma_{2}\right)
$$

and for each $k \in\{1,2, \ldots, n\}$ set

$$
T_{k}=\prod_{i=1}^{k}\left(1+\sigma_{i}\right) \prod_{j=k+1}^{n}\left(1-\sigma_{j}\right) \text { and } p=\prod_{1 \leq i \leq j \leq n}\left(\varepsilon_{i}-\varepsilon_{j}\right) .
$$

Lemma. (i) $S p$ is divisible by $\varepsilon_{1}^{2}-\varepsilon_{2}^{2}$.

(ii) Ap is divisible by $\varepsilon_{1} \varepsilon_{2}\left(\varepsilon_{1}^{2}-\varepsilon_{2}^{2}\right)$.

(iii) Take $k=[(n+1) / 2]$. Then

$$
T_{k} p=\prod_{1 \leq i \leq j \leq k}\left(\varepsilon_{i}^{2}-\varepsilon_{j}^{2}\right) \prod_{k+1 \leq r<s \leq n}\left(\varepsilon_{r}^{2}-\varepsilon_{s}^{2}\right) \prod_{t=k+1}^{n} \varepsilon_{t}
$$

up to a nonzero scalar. 
(i) Define the involution $s_{1}$ on $\mathbf{C}\left[\varepsilon_{1}, \varepsilon_{2}, \ldots, \varepsilon_{n}\right]$ through $s_{1} \varepsilon_{1}=\varepsilon_{2}, s_{1} \varepsilon_{2}=$ $\varepsilon_{1}, s_{1} \varepsilon_{j}=\varepsilon_{j}: j>2$. Given $q \in \mathbf{C}\left[\varepsilon_{1}, \varepsilon_{2}, \ldots, \varepsilon_{n}\right]$ one checks that $s_{1} q=-q$ implies that $\varepsilon_{1}-\varepsilon_{2}$ divides $q$. Clearly $s_{1} p=-p$. Since $s_{1}$ commutes with $S$, we conclude that $\varepsilon_{1}-\varepsilon_{2}$ divides $S p$. Yet $S p$ is an even polynomial in $\varepsilon_{1} \varepsilon_{2}$ and so $\varepsilon_{1}+\varepsilon_{2}$ also divides $S p$. The assertion follows by unique factorization.

(ii) Since $A p$ is odd in both $\varepsilon_{1}, \varepsilon_{2}$ it follows that $\varepsilon_{1}, \varepsilon_{2}$ both divide $A p$. We conclude as in (i).

(iii) Assume $n \geq 4$, so $k, n-k \geq 2$. Since the $\sigma_{i}$ commute pairwise, it follows from (i), (ii) and unique factorization that the right-hand side divides $T_{k} p$. Yet both sides have the same degree so it remains to prove that the scalar is nonzero. (This is a delicate point!) A standard expansion of $p$ gives a monomial of the form $\prod_{i=1}^{n} \varepsilon_{i}^{i-1}$ which is odd with respect to exactly $k=$ $[(n+1) / 2]$ involutions $\sigma_{i}$ and even with respect to the remaining $n-k$. Since $p$ is totally antisymmetric with respect to the permutation group $S_{n}$ on $n$ letters we can relabel indices to obtain a monomial in $p$ which is left invariant by $T_{k}$. This monomial cannot cancel with any of the other monomials because the $\sigma_{i}$ only change signs. This proves the required observation for $n \geq 4$. One checks the cases $n=2,3$ by hand.

6.7. The following result was reported by Macdonald [30, §3]. As his analysis is rather brief we have supplied a few extra details.

Corollary. Take $R$ of type $B_{n}$. If $R^{\prime}$ is of type $A_{2 k-2}: 2 k \leq n+1$ (resp. $\left.A_{2 k-1}: 2 k \leq n\right)$ and $R^{\prime \prime}$ is of type $D_{k} \times B_{k-1}$ (resp. $\left.D_{k} \times B_{k}\right)$ then

$$
j_{W^{\prime}}^{W}(s n) \cong j_{W^{\prime \prime}}^{W}(s n) .
$$

Both parts are similar and we prove only the first. Induction by stages reduces us to the case when $n=2 k-1$. We obtain an $A_{2 k-2}$ system $R^{\prime}$ in $B_{n}$ by suppressing the last root $\alpha_{n}$ and a $D_{k} \times B_{k-1}$ system by Dynkin's procedure of adding the negative $\alpha_{0}$ of the highest root and suppressing $\alpha_{k}$. Then using the Bourbaki notation for the roots of $B_{n}$ we find (notation 6.5) that $p$ spans the univalent module $M^{\prime}$ for the sign representation of $W^{\prime}$ while the right-hand side of 6.6(iii) spans the univalent module $M^{\prime \prime}$ for the sign representation of $W^{\prime \prime}$. (Both terms are the appropriate products of positive roots.) The assertion in 6.6(iii) implies that $M^{\prime \prime} \subset \mathrm{C} W M^{\prime}$ and so $\mathrm{C} W M^{\prime \prime}=\mathrm{C} W M^{\prime}$ by simplicity. Hence the assertion of the lemma.

6.8. By duality the above result holds for type $C$ also. Via 6.4 a similar conclusion holds for type $D$. Combined with 6.5 we obtain a number of nontrivial occurrences of $6.5(*)$ in particular all those used in constructing our table.

6.9. Take $R$ of type $B_{n}$ and $3 k+1 \leq n$. The subsystem $R^{\prime}$ obtained by suppressing $\alpha_{2 k+1}$ is of type $A_{2 k} \times B_{n-2 k-1}$. Via $6.5,6.7$ we have

$$
j_{W^{\prime}}^{W}(s n) \cong \tau\left(D_{k+1} \times B_{k} \times B_{n-2 k-1}\right)=E_{\alpha, \beta}
$$



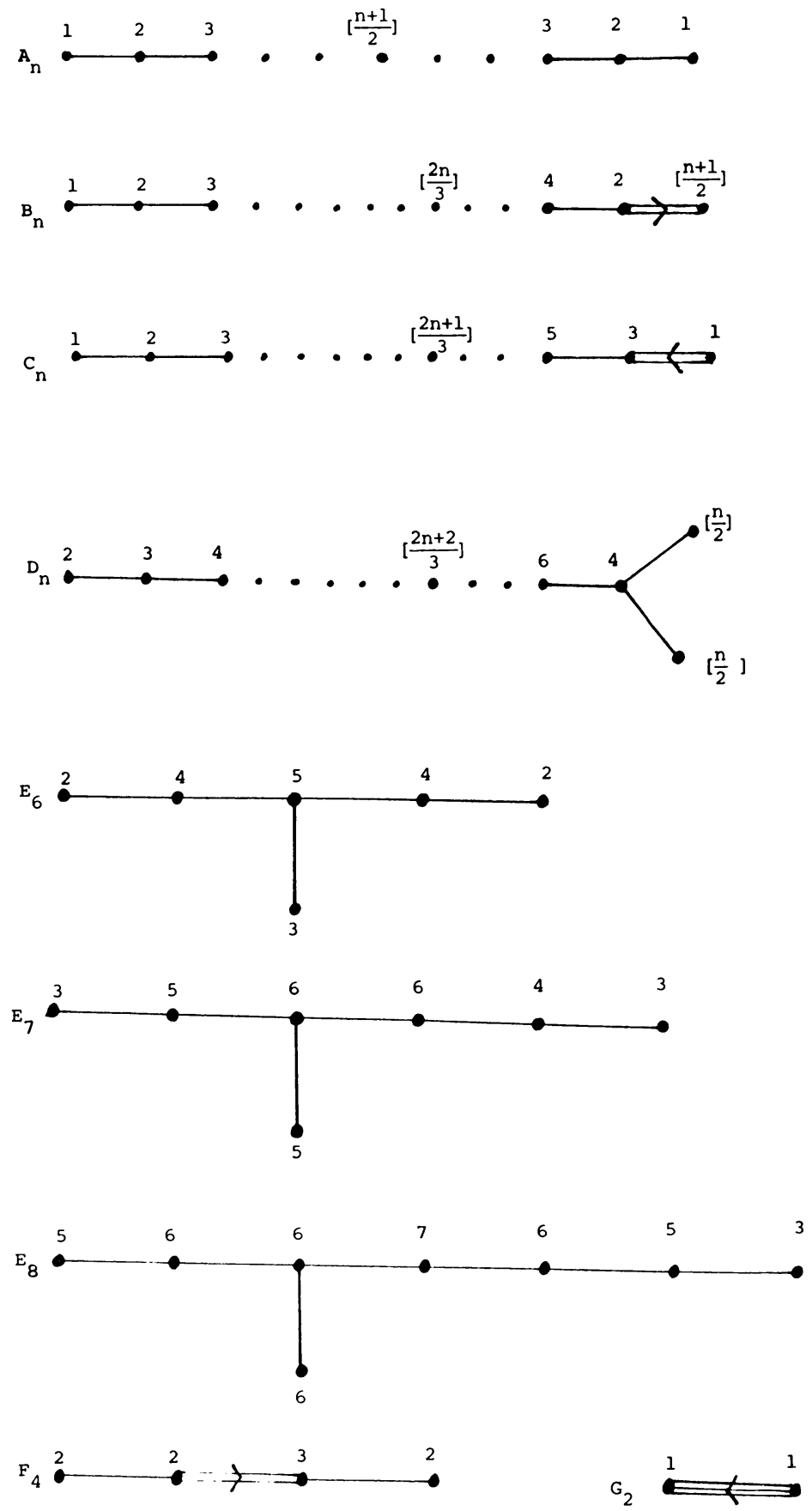

FIGURE

We depict for each classical Lie algebra the largest value reached by the multiplicity. In types $B_{n}, C_{n}, D_{n}$ the multiplicity increases by one on going from left to right and by two on going from right to left, the second operation being done last. (For greater precision, see table.) 
where $\alpha^{*}=(k+1), \beta^{*}=(k, n-2 k-1)$. We conclude that $m(\alpha)=0$, $m(\beta)=k$. Hence

$$
l_{\tau\left(R^{\prime}\right) \otimes s n}=3 k+1 .
$$

This agrees with the result tabulated under "degree".

6.10. Take $R$ of type $D_{n}$ and $3 k+1<n$. The subsystem $R^{\prime}$ obtained by suppressing $\alpha_{2 k+1}$ is of type $A_{2 k} \times D_{n-2 k-1}$. Via 6.5-6.8 we have

$$
j_{W^{\prime}}^{W}(s n) \cong E_{\alpha, \beta}
$$

where $\alpha^{*}=(k+1, n-2 k-1), \beta^{*}=k$. We conclude that $m(\alpha)=k+1$, $m(\beta)=0$ and $\inf (|\alpha|,|\beta|)=\inf (n-k, k)=k$. Hence

$$
l_{\tau\left(R^{\prime}\right) \otimes s n}=3 k+2
$$

as tabulated.

6.11. Finally the representation $E$ computed in say $6.9,6.10$ is related to that given under the heading "representation" by the operation $E \rightarrow E \otimes s n$ (in virtue of equality in 3.10 ). Here one must use Lusztig's procedure [26, I, $\S 5]$ to make the correspondence between the notation in the table and the classification given in 6.4-6.8 for the classical Lie algebras. For example in type $B_{n}$ the representation defined by $E_{\alpha, \beta}$ is described in the table by the array

$$
\left(\begin{array}{c}
\lambda_{1} \lambda_{2} \cdots \lambda_{m+1} \\
\mu_{1} \mu_{2} \cdots \mu_{m}
\end{array}\right)
$$

where $\lambda_{i}=\alpha_{i}+i-1, \mu_{j}=\beta_{j}+j-1$. For example take $\alpha=\alpha_{2 m+1}$ with $3 m+1<n$. Then $\left(\begin{array}{c}m n-2 m \\ m+1\end{array}\right)$ occurs under the heading representation and corresponds to $E_{(m, n-2 m-1),(m+1)}$. This is just $s n \otimes \tau\left(R^{\prime}\right)$ where $R^{\prime}$ is obtained by suppressing $\alpha_{2 m+1}$, as required. It also corresponds to $\tau\left(B^{\prime \prime}\right)$ where (see subtype) $B^{\prime \prime}$ is of type $A_{2}^{m} \times A_{1}$. Via 6.5 and 6.7 this is equivalent to $\tau\left(R_{1}\right)$ with $R_{1}$ a system of type $D_{1}^{n-3 m-1} \times D_{2}^{m} \times B_{1}^{m+1}$ and (taking dual partitions) to $E_{(m, n-2 m-1),(m+1)}$ as required.

\section{Numbered DyNKIN DiAgrams}

The multiplicities for corner weights are conveniently summarized by the numbered Dynkin diagrams shown in the figure above.

The table describing our computations for the classical (resp. exceptional) Lie algebras has been broken up over several pages with ony the first column repeated on each page. 
Table: Notation-Bourbaki [7], Carter [8], Lusztig [26, I].

Classical Lie Algebras

\begin{tabular}{|c|c|c|c|}
\hline Type & Root & Coefficient & $B_{0}$ \\
\hline $\begin{array}{c}A_{n} \\
n \text { odd } \\
\end{array}$ & $\alpha_{m}: 1 \leq m \leq \frac{n+1}{2}$ & $-(n-1)$ & $\varepsilon_{i}-\varepsilon_{i+\frac{n-1}{2}}: 1 \leq i \leq m$ \\
\hline $\begin{array}{c}A_{n} \\
n \text { even }\end{array}$ & $\alpha_{m}: 1 \leq m \leq \frac{n}{2}$ & $-(n-1)$ & \\
\hline$B_{n}$ & $\begin{array}{c}\alpha_{2 m+1} \\
1 \leq 3 m+1<n\end{array}$ & $-2(n-m-1)$ & $\varepsilon_{m \pm i+1} \pm \varepsilon_{n-i+1}: 1 \leq i \leq m$ \\
\hline$B_{n}$ & $3 m+1 \stackrel{\alpha_{2 m+1}}{\geq n>2 m+1}$ & $-2(n-m-1)$ & $\begin{array}{c}\varepsilon_{m \pm i+1} \pm \varepsilon_{n-i+1}: 1 \leq i \leq n-2 m-1 \\
\varepsilon_{m-j+1}-\varepsilon_{n-j+1}: n-2 m \leq j \leq m \\
\varepsilon_{m+1}\end{array}$ \\
\hline$B_{n}$ & $3 \leq \begin{array}{l}\alpha_{2 m} m \\
3 m\end{array} \leq n$ & $-2(n-m-1)-1$ & $\varepsilon_{m-i+1}+\varepsilon_{m+i}: 1 \leq i \leq m$ \\
\hline$B_{n}$ & $\begin{array}{c}\alpha_{2 m} \\
3 m>n>2 m\end{array}$ & $-2(n-m-1)-1$ & $\varepsilon_{m-i+1}+\varepsilon_{m+i}: 1 \leq i \leq m$ \\
\hline $\begin{array}{c}B_{n} \\
n \text { odd }\end{array}$ & $\alpha_{n}$ & $-(n-1)$ & $\begin{array}{c}\varepsilon_{i}+\varepsilon_{n-i+1}: 1 \leq i \leq \frac{n-1}{2} \\
\varepsilon_{\frac{n+1}{2}}\end{array}$ \\
\hline $\begin{array}{c}B_{n} \\
n \text { even }\end{array}$ & $\alpha_{n}$ & $-(n-1)$ & $\varepsilon_{i}+\varepsilon_{n-i+1}: 1 \leq i \leq \frac{n}{2}$ \\
\hline$C_{n}$ & $\begin{array}{c}\alpha_{2 m+1} \\
1 \leq 3 m+1<n \\
\end{array}$ & $-2(n-m-2)-1$ & $\varepsilon_{m-i+2}+\varepsilon_{m+i}: 1 \leq i \leq m+1$ \\
\hline$C_{n}$ & $3 m+1 \stackrel{\alpha_{2 m+1}}{\geq} n>2 m+1$ & $-2(n-m-2)-1$ & $\varepsilon_{m-i+2}+\varepsilon_{m+i}: 1 \leq i \leq m+1$ \\
\hline$C_{n}$ & $3 \leq \begin{array}{l}\alpha_{2 m} \\
3 m\end{array} \leq n$ & $-2(n-m-1)$ & $\left.\begin{array}{c}\varepsilon_{m-i+1}-\varepsilon_{n-i+1} \\
\varepsilon_{m+i}+\varepsilon_{n-i+1}\end{array}\right\}: 1 \leq i \leq m$ \\
\hline$C_{n}$ & $\begin{array}{c}\alpha_{2 m} \\
3 m>n>2 m\end{array}$ & $-2(n-m-1)$ & 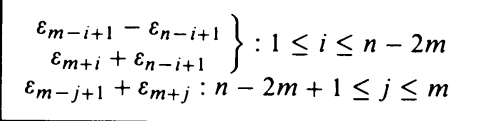 \\
\hline $\begin{array}{c}C_{n} \\
n \text { odd }\end{array}$ & $\alpha_{n}$ & $-2(n-1)$ & $\varepsilon_{i}+\varepsilon_{n-i+1}: 1 \leq i \leq \frac{n+1}{2}$ \\
\hline $\begin{array}{c}C_{n} \\
n \text { even }\end{array}$ & $\alpha_{n}$ & $-2(n-1)$ & $\varepsilon_{i}+\varepsilon_{n-i+1}: 1 \leq i \leq \frac{n}{2}$ \\
\hline$D_{n}$ & $\begin{array}{c}\alpha_{2 m+1} \\
1 \leq 3 m+1<n\end{array}$ & $-2(n-m-2)$ & $\begin{array}{c}\varepsilon_{m+1} \pm \varepsilon_{n} \\
\varepsilon_{m \pm i+1} \pm \varepsilon_{n-i}: 1 \leq i \leq m\end{array}$ \\
\hline$D_{n}$ & $3 m+1 \stackrel{\alpha_{2 m+1}}{\geq} n>2 m+1$ & $-2(n-m-2)$ & $\begin{array}{c}\varepsilon_{m+1} \pm \varepsilon_{n} \\
\varepsilon_{m \pm i+1} \pm \varepsilon_{n-i}: 1 \leq i \leq n-2 m-2 \\
\varepsilon_{m-j+1}+\varepsilon_{m+j+1}: n-2 m-1 \leq j \leq m\end{array}$ \\
\hline$D_{n}$ & $\begin{array}{c}\alpha_{2 m} \\
3 \leq 3 m<n\end{array}$ & $-2(n-m-2)-1$ & $\varepsilon_{m-i+1}+\varepsilon_{m+i}: 1 \leq i \leq m$ \\
\hline$D_{n}$ & $\begin{array}{c}\alpha_{2 m} \\
3 m \geq n>2 m+1\end{array}$ & $-2(n-m-2)-1$ & $\varepsilon_{m-i+1}+\varepsilon_{m+i}: 1 \leq i \leq m$ \\
\hline $\begin{array}{c}D_{n} \\
n \text { odd }\end{array}$ & $\alpha_{n-1}, \alpha_{n}$ & $-2(n-2)$ & $\begin{array}{c}\varepsilon_{1}-\varepsilon_{n} \\
\varepsilon_{i+1}+\varepsilon_{n-i}: 1 \leq i \leq \frac{n-3}{2}\end{array}$ \\
\hline $\begin{array}{c}D_{n} \\
n \text { even }\end{array}$ & $\alpha_{n-1}, \alpha_{n}$ & $-2(n-2)$ & $\begin{array}{c}\varepsilon_{1}-\varepsilon_{n} \\
\varepsilon_{i+1}+\varepsilon_{n-i}: 1 \leq i \leq \frac{n-2}{2}\end{array}$ \\
\hline
\end{tabular}


Classical Lie Algebras

\begin{tabular}{|c|c|c|c|}
\hline Type & $B_{1}$ & Orbit $(1)=G X$ & Orbit $(2)=G x$ \\
\hline $\begin{array}{c}A_{n} \\
n \text { odd }\end{array}$ & & $(n-m+1, m)$ & $(n-m+1, m)$ \\
\hline $\begin{array}{c}A_{n} \\
n \text { even }\end{array}$ & $\left.\begin{array}{r}-\left(\varepsilon_{i}-\varepsilon_{\left.i+\frac{n-2}{2}\right)}\right. \\
\varepsilon_{i}-\varepsilon_{i+\frac{n}{2}}\end{array}\right\}: 1 \leq i \leq n$ & $(n-m+1, m)$ & $(n-m+1, m)$ \\
\hline$B_{n}$ & & $(2 m+1 ; 2 n-4 m-1)$ & $(2 m+1 ; 2 n-4 m-1)$ \\
\hline$B_{n}$ & & $(2 m+1 ; 2 n-4 m-1)$ & $(2 m+1 ; 2 n-4 m-1)$ \\
\hline$B_{n}$ & $\begin{array}{c}-\left(\varepsilon_{m-i+1}-\varepsilon_{n-i+1}\right): 1 \leq i \leq m \\
-\left(\varepsilon_{m+j+1}-\varepsilon_{n-j+1}\right): 1 \leq j \leq m-1 \\
-\varepsilon_{m+1}\end{array}$ & $(2 m ; 2 n-4 m-1)$ & $(2 n ; 2 n-4 m-1)$ \\
\hline$B_{n}$ & $\begin{array}{c}-\left(\varepsilon_{m \pm i+1} \pm \varepsilon_{n-i+1}\right): 1 \leq i \leq n-2 m \\
-\varepsilon_{m+1}\end{array}$ & $(2 m ; 2 n-4 m-1)$ & $(\varnothing ; 2 m+1,2 m-1,2 n-4 m+1)$ \\
\hline $\begin{array}{l}B_{n} \\
n \text { odd }\end{array}$ & & $(n ; 1)$ & $(n ; 1)$ \\
\hline $\begin{array}{c}B_{n} \\
n \text { even } \\
\end{array}$ & $-\varepsilon_{\frac{n}{2}}+1$ & $(n ; 1)$ & $(\varnothing ; n+1, n-1,1)$ \\
\hline$C_{n}$ & $\begin{array}{c}-\left(\varepsilon_{m-i+2}-\varepsilon_{n-i+1}\right): 1 \leq i \leq m+1 \\
-\left(\varepsilon_{m+j+1}-\varepsilon_{n-j+1}\right): 1 \leq j \leq m \\
\end{array}$ & $(2 m+1 ; n-2 m-1)$ & $(\varnothing ; n-2 m-1, m+1, m)$ \\
\hline$C_{n}$ & $\left.\begin{array}{r}-\left(\varepsilon_{m-i+2}-\varepsilon_{n-i+1}\right) \\
-\left(\varepsilon_{m+i+1}+\varepsilon_{n-i+1}\right)\end{array}\right\}: 1 \leq i \leq \eta-2 m-1$ & $(2 m+1 ; n-2 m-1)$ & $(2 m+1 ; n-2 m-1)$ \\
\hline$C_{n}$ & & $(2 m ; n-2 m)$ & $(2 m ; n-2 m)$ \\
\hline$C_{n}$ & & $(2 m ; n-2 m)$ & $(2 m ; n-2 m)$ \\
\hline $\begin{array}{l}C_{n} \\
n \text { odd }\end{array}$ & & $(n ; \varnothing)$ & $(n ; \varnothing)$ \\
\hline $\begin{array}{c}C_{n} \\
n \text { even }\end{array}$ & & $(n ; \varnothing)$ & $(n ; \varnothing)$ \\
\hline$D_{n}$ & & $(2 m+1 ; 2 n-4 m-3,1)$ & $(2 m+1 ; 2 n-4 m-3,1)$ \\
\hline$D_{n}$ & & $(2 m+1 ; 2 n-4 m-3,1)$ & $(2 m+1 ; 2 n-4 m-3,1)$ \\
\hline$D_{n}$ & & $(2 m ; 2 n-4 m-1,1)$ & $(2 n-4 m-1,2 m+1 ; 2 m-1,1)$ \\
\hline$D_{n}$ & $\begin{array}{c}-\left(\varepsilon_{m+1} \pm \varepsilon_{n}\right) \\
-\left(\varepsilon_{m-i+1}-\varepsilon_{n-i}\right): 1 \leq i \leq m \\
-\left(\varepsilon_{m+i+1}-\varepsilon_{n-i}: 1 \leq i \leq m-1\right. \\
\end{array}$ & $(2 m ; 2 n-4 m-1,1)$ & $(2 m ; 2 n-4 m-1,1)$ \\
\hline$\underline{D_{n}}$ & $\begin{array}{c}-\left(\varepsilon_{m+1} \pm \varepsilon_{n}\right) \\
-\left(\varepsilon_{m \pm i+1} \pm \varepsilon_{n-i}\right): 1 \leq i \leq n-2 m-1\end{array}$ & $(1 ; n, n-2)$ & $(1 ; n, n-2)$ \\
\hline $\begin{array}{l}D_{n} \\
n \text { odd } \\
\end{array}$ & & $(1 ; n+1, n-3)$ & $(1 ; n+1, n-3)$ \\
\hline $\begin{array}{l}D_{n} \\
n \text { even }\end{array}$ & & $(1 ; n+1, n-3)$ & $(1 ; n+1, n-3)$ \\
\hline
\end{tabular}




\section{Classical Lie Algebras}

\begin{tabular}{|c|c|c|c|}
\hline Type & degree & Invariant parabolic $\tilde{B}^{\prime \prime}$ & $B^{\prime \prime}$ \\
\hline $\begin{array}{c}A_{n} \\
n \text { odd }\end{array}$ & $m$ & $B_{0}$ & $\alpha_{\frac{n-1}{2}-m+2 i}: 1 \leq i \leq m$ \\
\hline $\begin{array}{c}A_{n} \\
n \text { even }\end{array}$ & $m$ & $\varepsilon_{i}-\varepsilon_{i+\frac{n-2}{n}}: 1 \leq i \leq m$ & $\alpha_{\frac{n-2}{2}-m+2 i}: 1 \leq i \leq m$ \\
\hline$B_{n}$ & $3 m+1$ & $B_{0}$ & $\frac{\alpha_{n}}{\alpha_{n-3(m-i+1)}, \alpha_{n-3(m-i+1)+1}: 1 \leq i \leq m}$ \\
\hline$B_{n}$ & $2 n-3 m-1$ & $B_{0}$ & $\begin{array}{c}\alpha_{n} \\
\alpha_{2 i-1}: 1 \leq i \leq 3 m-n+1 \\
\alpha_{6 m-2 n+3 j}, \alpha_{6 m-2 n+3 j+1}: 1 \leq j \leq n-2 m-1\end{array}$ \\
\hline$B_{n}$ & $3 m$ & $\left.\begin{array}{c}\varepsilon_{m-i+1}-\varepsilon_{n-i+1} \\
\varepsilon_{m+i}-\varepsilon_{n-i+1}\end{array}\right\}: 1 \leq i \leq m$ & $\alpha_{n-3(m-i+1)+1}, \alpha_{n-3(m-i+1)+2}: 1 \leq i \leq m$ \\
\hline$B_{n}$ & $2 n-3 m$ & $\begin{array}{c}\varepsilon_{m-i+1}+\varepsilon_{m+i+1}: n-2 m+1 \leq i \leq m-1 \\
\varepsilon_{m \pm j+1} \pm \varepsilon_{n-j+1}: 1 \leq j \leq n-2 m \\
\varepsilon_{m+1}\end{array}$ & $\begin{array}{c}\alpha_{2 i}: 1 \leq i \leq 3 m-n-1 \\
\alpha_{6 m-2 n-3+3 j}, \alpha_{6 m-2 n-2+3 j}: 1 \leq j \leq n-2 m \\
\alpha_{n}\end{array}$ \\
\hline $\begin{array}{c}B_{n} \\
n \text { odd }\end{array}$ & $\frac{n+1}{2}$ & $B_{0}$ & $\alpha_{n+2-2 i}: 1 \leq i \leq \frac{n+1}{2}$ \\
\hline $\begin{array}{c}B_{n} \\
n \text { even }\end{array}$ & $\frac{n}{2}$ & $\begin{array}{c}\varepsilon_{i+1}+\varepsilon_{n-i+1}: 1 \leq i \leq \frac{n-2}{2} \\
\varepsilon_{\frac{n}{2}+1}\end{array}$ & $\alpha_{n+2-2 i}: 1 \leq i \leq \frac{n}{2}$ \\
\hline$C_{n}$ & $3 m+1$ & $\left\{-\alpha: \alpha \in B_{1}\right\}$ & $\begin{array}{c}\alpha_{n-3 m-1} \\
\alpha_{n-3(m-i)-2}, \alpha_{n-3(m-i)-1}: 1 \leq i \leq m\end{array}$ \\
\hline$C_{n}$ & $2 n-3 m-1$ & $\begin{array}{r}\varepsilon_{m-i+1}+\varepsilon_{m+i+1}: n-2 m \leq i \leq m-1 \\
2 \varepsilon_{m+1} \\
\varepsilon_{m \pm j+1} \pm \varepsilon_{n-j+1}: 1 \leq j \leq n-2 m-1\end{array}$ & $\begin{array}{c}\alpha_{n} \\
\alpha_{2 i-1}: 1 \leq i \leq 3 m-n+1 \\
\alpha_{6 m-2 n+3 j}, \alpha_{6 m-2 n+3 j+1}: 1 \leq j \leq n-2 m-1\end{array}$ \\
\hline$C_{n}$ & $3 m$ & $B_{0}$ & $\alpha_{n-3(m-i+1)+1}, \alpha_{n-3(m-i+1)+2}: 1 \leq i \leq m$ \\
\hline$C_{n}$ & $2 n-3 m$ & $B_{0}$ & $\begin{array}{c}\alpha_{2 i-1}: 1 \leq i \leq 3 m-n \\
\alpha_{6 m-2 n+3 j-2}, \alpha_{6 m-2 n+3 j-1}: 1 \leq j \leq n-2 m\end{array}$ \\
\hline $\begin{array}{c}C_{n} \\
n \text { odd }\end{array}$ & $\frac{n+1}{2}$ & $B_{0}$ & $\alpha_{2 i-1}: 1 \leq i \leq \frac{n+1}{2}$ \\
\hline $\begin{array}{l}C_{n} \\
n \text { even }\end{array}$ & $\frac{n}{2}$ & $B_{0}$ & $\alpha_{2 i-1}: 1 \leq i \leq \frac{n}{2}$ \\
\hline$D_{n}$ & $3 m+2$ & $B_{0}$ & $\begin{array}{c}\alpha_{n-1}, \alpha_{n} \\
\alpha_{n-3(m-i+1)-1}, \alpha_{n-3(m-i+1)}: 1 \leq i \leq m \\
\end{array}$ \\
\hline$D_{n}$ & $2 n-3 m-2$ & $B_{0}$ & 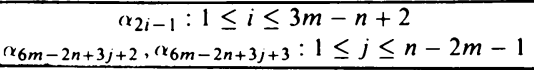 \\
\hline$D_{n}$ & $3 m$ & $\begin{array}{c}\varepsilon_{1}-\varepsilon_{n-m}, \varepsilon_{m+1} \pm \varepsilon_{n} \\
\varepsilon_{m \pm i+1} \pm \varepsilon_{n-i}: 1 \leq i \leq m-1 \\
\end{array}$ & $\begin{array}{c}\alpha_{n-3 m}, \alpha_{n-1}, \alpha_{n} \\
\alpha_{n-3(m-i+1)+2}, \alpha_{n-3(m-i+1)+3}: 1 \leq i \leq m-1\end{array}$ \\
\hline$D_{n}$ & $2 n-3 m$ & 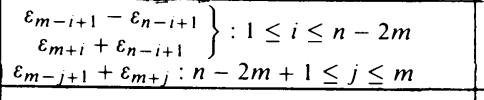 & $\begin{array}{c}\alpha_{2 i-1}: 1 \leq i \leq 3 m-n \\
\alpha_{6 m-2 n+3 j-2}, \alpha_{6 m-2 n+3 j-1}: 1 \leq j \leq n-2 m\end{array}$ \\
\hline $\begin{array}{l}D_{n} \\
n \text { odd }\end{array}$ & $\frac{n-1}{2}$ & $B_{0}$ & $\alpha_{2 i+2}: 1 \stackrel{\alpha_{n}}{\leq} i \leq \frac{n-3}{2}$ \\
\hline $\begin{array}{c}D_{n} \\
n \text { even }\end{array}$ & $\frac{n}{2}$ & $B_{0}$ & $\alpha_{2 i+1}: 1 \stackrel{\alpha_{n}}{\leq} i \leq \frac{n-2}{2}$ \\
\hline
\end{tabular}




\section{Classical Lie Algebras}

\begin{tabular}{|c|c|c|c|c|c|c|}
\hline Type & $\operatorname{dim} V_{B^{\prime \prime}}(\mu)$ & Subtype & $\left|A_{x}\right|$ & Representation & length & $\tilde{B}^{\prime \prime \prime}$ \\
\hline $\begin{array}{c}A_{n} \\
n \text { odd }\end{array}$ & 1 & $A_{1}^{m}$ & & & & \\
\hline $\begin{array}{c}A_{n} \\
n \text { even } \\
\end{array}$ & 1 & $A_{1}^{m}$ & & & & \\
\hline$B_{n}$ & 2 & $A_{2}^{m} \times A_{1}$ & 2 & $\left(\begin{array}{c}m n-2 m \\
m+1\end{array}\right)$ & 2 & $\left.\begin{array}{c}\varepsilon_{1}+\varepsilon_{2 m+2} \\
\varepsilon_{m+i+1}+\varepsilon_{n-i+1} \\
\varepsilon_{m-i+2}-\varepsilon_{n-i+1}\end{array}\right\}: 1 \leq i \leq m$ \\
\hline$B_{n}$ & $2^{3 m-n+2}$ & $A_{2}^{n-2 m-1} \times A_{1}^{3 m-n+2}$ & 2 & $\left(\begin{array}{c}n-2 m-1 m+1 \\
m+1\end{array}\right)$ & 1 & \\
\hline$B_{n}$ & 1 & $A_{2}^{m}$ & 1 & $\left(\begin{array}{c}m n-2 m+1 \\
m\end{array}\right)$ & 1 & \\
\hline$B_{n}$ & $2^{3 m-n}$ & $A_{2}^{n-2 m} \times A_{1}^{3 m-n}$ & 4 & $\left(\begin{array}{c}n-2 m m+1 \\
m\end{array}\right)$ & 2 & $\left.\begin{array}{c}\varepsilon_{m-i+1}+\varepsilon_{m+i}: n-2 m+1 \leq i \leq m \\
\varepsilon_{m-j+1}-\varepsilon_{n-j+1} \\
\varepsilon_{m+j}+\varepsilon_{n-j+1}\end{array}\right\}: 1 \leq j \leq n-2 m$ \\
\hline $\begin{array}{c}B_{n} \\
n \text { odd } \\
\end{array}$ & 1 & $A_{1}^{\frac{n+1}{2}}$ & & & & \\
\hline $\begin{array}{c}B_{n} \\
n \text { even }\end{array}$ & 1 & $A_{1}^{\frac{n}{2}}$ & & & & \\
\hline$C_{n}$ & 1 & $A_{2}^{m} \times A_{1}$ & 4 & $\left(\begin{array}{c}m n-2 m \\
m+1\end{array}\right)$ & 2 & \\
\hline$C_{n}$ & $2^{3 m-n+1}$ & $A_{2}^{n-2 m-1} \times A_{1}^{3 m-n+2}$ & 1 & $\left(\begin{array}{c}n-2 m-1 \\
m+1\end{array}\right)$ & 1 & \\
\hline$C_{n}$ & 1 & $A_{2}^{m}$ & 2 & $\left(\begin{array}{c}m n-2 m+1 \\
m\end{array}\right)$ & 2 & \\
\hline$C_{n}$ & $2^{3 m-n}$ & $A_{2}^{n-2 m} \times A_{1}^{3 m-n}$ & 2 & $\left(\begin{array}{c}n-2 m \\
m\end{array}{ }^{m+1}\right)$ & 2 & $\begin{array}{c}2 \varepsilon_{m+1} \\
\varepsilon_{m-i+1}+\varepsilon_{m+i}: n-2 m+2 \leq i \leq m \\
\varepsilon_{m \pm j+1} \pm \varepsilon_{n-j+1}: 1 \leq j \leq n-2 m \\
\end{array}$ \\
\hline $\begin{array}{c}C_{n} \\
n \text { odd }\end{array}$ & $2^{\frac{n-1}{2}}$ & $A_{1}^{\frac{n+1}{2}}$ & 1 & $\left(\begin{array}{c}0 \frac{n+1}{2} \\
\frac{n+1}{2}\end{array}\right)$ & 1 & \\
\hline $\begin{array}{c}C_{n} \\
n \text { even }\end{array}$ & $2^{\frac{n}{2}}$ & $A_{1}^{\frac{n}{2}}$ & 2 & $\left(\begin{array}{c}0 \frac{n+2}{2} \\
\frac{n}{2}\end{array}\right)$ & 2 & $\varepsilon_{i+1}+\varepsilon_{n-i+1}: 1 \leq i \leq \frac{n}{2}$ \\
\hline$D_{n}$ & 1 & $A_{2}^{m} \times A_{1}^{2}$ & & & & \\
\hline$D_{n}$ & $2^{3 m-n+2}$ & $A_{2}^{n-2 m-2} \times A_{1}^{3 m-n+4}$ & 2 & $\left(\begin{array}{c}0 m+1 \\
n-2 m+1 m+2\end{array}\right)$ & 2 & $\left.\begin{array}{c}\varepsilon_{m-j+1}+\varepsilon_{m+j+1}: n-2 m \leq j \leq m \\
\varepsilon_{m-i+1}-\varepsilon_{n-i} \\
\varepsilon_{m+i+2}+\varepsilon_{n-i}\end{array}\right\}: 0 \leq i \leq n-2 m-2$ \\
\hline$D_{n}$ & 1 & $A_{2}^{m-1} \times A_{1}^{3}$ & & & & \\
\hline$D_{n}$ & $2^{3 m-n}$ & $A_{2}^{n-2 m} \times A_{1}^{3 m-n}$ & 1 & $\left(\begin{array}{c}0 m+1 \\
n-2 m m+1\end{array}\right)$ & 1 & \\
\hline $\begin{array}{c}D_{n} \\
n \text { odd } \\
\end{array}$ & 1 & $A_{1}^{\frac{n-1}{2}}$ & & & & \\
\hline $\begin{array}{c}D_{n} \\
n \text { even }\end{array}$ & 1 & $A_{1}^{\frac{n}{2}}$ & & & & \\
\hline
\end{tabular}




\section{Classical Lie Algebras}

\begin{tabular}{|c|c|c|c|}
\hline Type & $B^{\prime \prime \prime}$ & $\operatorname{dim} V_{B^{\prime \prime \prime}}\left(\mu^{\prime}\right)$ & Multiplicity \\
\hline $\begin{array}{c}A_{n} \\
n \text { odd }\end{array}$ & & & $m$ \\
\hline $\begin{array}{c}A_{n} \\
n \text { even } \\
\end{array}$ & & & $m$ \\
\hline$B_{n}$ & $\left.\begin{array}{c}\alpha_{n-3 m-1} \\
\alpha_{n-3 m-2+3 i} \\
\alpha_{n-3 m-1+3 i}\end{array}\right\}: 1 \leq i \leq m$ & 1 & $2 m+1$ \\
\hline$B_{n}$ & & & $2 n-4 m-2$ \\
\hline$B_{n}$ & & & $2 m$ \\
\hline$B_{n}$ & $\left.\begin{array}{c}\alpha_{2 i-1}: 1 \leq i \leq 3 m-n \\
\alpha_{6 m-2 n-2+3 j} \\
\alpha_{6 m-2 n-1+3 j}\end{array}\right\}: 1 \leq j \leq n-2 m$ & $2^{3 m-n}$ & $2 n-4 m$ \\
\hline $\begin{array}{c}B_{n} \\
n \text { odd } \\
\end{array}$ & & & $\frac{n+1}{2}$ \\
\hline $\begin{array}{c}B_{n} \\
n \text { even }\end{array}$ & & & $\frac{n}{2}$ \\
\hline$C_{n}$ & & & $2 m+1$ \\
\hline$C_{n}$ & & & $2 n-4 m-1$ \\
\hline$C_{n}$ & & & $2 m$ \\
\hline$C_{n}$ & $\begin{array}{c}\alpha_{n} \\
\alpha_{2 i-1}: 1 \leq i \leq 3 m-n-1 \\
\alpha_{6 m-2 n+3 j-3}, \alpha_{6 m-2 n+3 j-2}: 1 \leq j \leq n-2 m\end{array}$ & $2^{3 m-n-1}$ & $2 n-4 m+1$ \\
\hline $\begin{array}{c}C_{n} \\
n \text { odd } \\
\end{array}$ & & & 1 \\
\hline $\begin{array}{c}C_{n} \\
n \text { even } \\
\end{array}$ & $\alpha_{2 i}: 1 \leq i \leq \frac{n}{2}$ & $2^{\frac{n-2}{2}}$ & 1 \\
\hline$D_{n}$ & & & $2 m+2$ \\
\hline$D_{n}$ & $\begin{array}{c}\alpha_{2 j-1}: 1 \leq j \leq 3 m-n+1 \\
\alpha_{6 m-2 n+3 i+4}, \alpha_{6 m-2 n+3 i+5}: 0 \leq i \leq n-2 m-2\end{array}$ & $2^{3 m-n+1}$ & $2 n-4 m-2$ \\
\hline$D_{n}$ & & & $2 m+1$ \\
\hline$D_{n}$ & & & $2 n-4 m$ \\
\hline $\begin{array}{c}D_{n} \\
n \text { odd } \\
\end{array}$ & & & $\frac{n-1}{2}$ \\
\hline $\begin{array}{c}D_{n} \\
n \text { even }\end{array}$ & & & $\frac{n}{2}$ \\
\hline
\end{tabular}




\section{Exceptional Lie Algebras}

\begin{tabular}{|c|c|c|c|}
\hline Type & Root & Coefficient & $B_{0}$ \\
\hline$E_{6}$ & $\alpha_{1}, \alpha_{6}$ & -10 & $\left(\begin{array}{c}11111 \\
1\end{array}\right),\left(\begin{array}{c}11210 \\
1\end{array}\right)$ \\
\hline$E_{6}$ & $\alpha_{2}$ & -9 & $\left(\begin{array}{c}12321 \\
2\end{array}\right)$ \\
\hline$E_{6}$ & $\alpha_{3}, \alpha_{5}$ & -7 & $\left(\begin{array}{c}12221 \\
1\end{array}\right)$ \\
\hline$E_{6}$ & $\alpha_{4}$ & -5 & $\left(\begin{array}{c}01221 \\
1\end{array}\right),\left(\begin{array}{c}11211 \\
1\end{array}\right),\left(\begin{array}{c}12210 \\
1\end{array}\right)$ \\
\hline$E_{7}$ & $\alpha_{1}$ & -15 & $\left(\begin{array}{c}234321 \\
2\end{array}\right)$ \\
\hline$E_{7}$ & $\alpha_{2}$ & -12 & $\left(\begin{array}{c}111111 \\
1\end{array}\right),\left(\begin{array}{c}012210 \\
1\end{array}\right),\left(\begin{array}{c}012111 \\
1\end{array}\right),\left(\begin{array}{c}122100 \\
1\end{array}\right),\left(\begin{array}{c}112110 \\
1\end{array}\right)$ \\
\hline$E_{7}$ & $\alpha_{3}$ & -9 & $\left(\begin{array}{c}123210 \\
2\end{array}\right),\left(\begin{array}{c}123211 \\
1\end{array}\right),\left(\begin{array}{c}122221 \\
1\end{array}\right)$ \\
\hline$E_{7}$ & $\alpha_{4}$ & -6 & $\left(\begin{array}{c}011100 \\
1\end{array}\right),\left(\begin{array}{c}001111 \\
0\end{array}\right),\left(\begin{array}{c}111000 \\
1\end{array}\right),\left(\begin{array}{c}011110 \\
0\end{array}\right) \cdot\left(\begin{array}{c}001110 \\
1\end{array}\right),\left(\begin{array}{c}111100 \\
0\end{array}\right)$ \\
\hline$E_{7}$ & $\alpha_{5}$ & -8 & $\left(\begin{array}{c}111100 \\
1\end{array}\right),\left(\begin{array}{c}011111 \\
0\end{array}\right),\left(\begin{array}{c}011110 \\
1\end{array}\right),\left(\begin{array}{c}012100 \\
1\end{array}\right),\left(\begin{array}{c}111110 \\
0\end{array}\right),\left(\begin{array}{c}001111 \\
1\end{array}\right)$ \\
\hline$\underline{E_{7}}$ & $\alpha_{6}$ & -11 & $\left(\begin{array}{c}123221 \\
2\end{array}\right),\left(\begin{array}{c}123321 \\
1\end{array}\right)$ \\
\hline$E_{7}$ & $\alpha_{7}$ & -16 & $\left(\begin{array}{c}122111 \\
1\end{array}\right),\left(\begin{array}{c}112211 \\
1\end{array}\right),\left(\begin{array}{c}012221 \\
1\end{array}\right)$ \\
\hline$E_{8}$ & $\alpha_{1}$ & -21 & $\left(\begin{array}{c}2454321 \\
2\end{array}\right),\left(\begin{array}{c}2354321 \\
3\end{array}\right)$ \\
\hline$E_{8}$ & $\alpha_{2}$ & -15 & $\left(\begin{array}{c}2343210 \\
2\end{array}\right),\left(\begin{array}{c}1343211 \\
2\end{array}\right),\left(\begin{array}{c}1243221 \\
2\end{array}\right),\left(\begin{array}{c}1233321 \\
2\end{array}\right)$ \\
\hline$E_{8}$ & $\alpha_{3}$ & -11 & $\left(\begin{array}{c}1232210 \\
1\end{array}\right),\left(\begin{array}{c}1232211 \\
1\end{array}\right),\left(\begin{array}{c}1222221 \\
1\end{array}\right),\left(\begin{array}{c}1232111 \\
2\end{array}\right),\left(\begin{array}{c}1233210 \\
1\end{array}\right)$ \\
\hline$E_{8}$ & $\alpha_{4}$ & -7 & $\left(\begin{array}{c}1122110 \\
1\end{array}\right),\left(\begin{array}{c}1121111 \\
1\end{array}\right),\left(\begin{array}{c}1222100 \\
1\end{array}\right),\left(\begin{array}{c}0122210 \\
1\end{array}\right),\left(\begin{array}{c}0122111 \\
1\end{array}\right),\left(\begin{array}{c}1221110 \\
1\end{array}\right)$ \\
\hline$E_{8}$ & $\alpha_{5}$ & -9 & $\left(\begin{array}{c}1232110 \\
1\end{array}\right),\left(\begin{array}{c}1122211 \\
1\end{array}\right),\left(\begin{array}{c}1222210 \\
1\end{array}\right),\left(\begin{array}{c}1232100 \\
2\end{array}\right),\left(\begin{array}{c}0122221 \\
1\end{array}\right),\left(\begin{array}{c}1222111 \\
1\end{array}\right)$ \\
\hline$E_{8}$ & $\alpha_{6}$ & -.12 & $\left(\begin{array}{c}0111111 \\
1\end{array}\right),\left(\begin{array}{c}1121100 \\
1\end{array}\right),\left(\begin{array}{c}1111110 \\
1\end{array}\right),\left(\begin{array}{c}0121110 \\
1\end{array}\right),\left(\begin{array}{c}1111111 \\
0\end{array}\right),\left(\begin{array}{c}0122100 \\
1\end{array}\right)$ \\
\hline$E_{8}$ & $\alpha_{7}$ & -17 & $\left(\begin{array}{c}2343221 \\
2\end{array}\right),\left(\begin{array}{c}1343321 \\
2\end{array}\right),\left(\begin{array}{c}1244321 \\
2\end{array}\right)$ \\
\hline$E_{8}$ & $\alpha_{8}$ & -27 & $\left(\begin{array}{c}2465432 \\
3\end{array}\right)$ \\
\hline$F_{4}$ & $\alpha_{1}$ & -9 & $(2342)$ \\
\hline$F_{4}$ & $\alpha_{2}$ & -5 & $(1222),(1234)$ \\
\hline$F_{4}$ & $\alpha_{3}$ & -3 & $(1220),(0122),(1121)$ \\
\hline$F_{4}$ & $\alpha_{4}$ & -6 & $(1111),(0121)$ \\
\hline$G_{2}$ & $\alpha_{1}$ & -1 & $2 \alpha_{1}+\alpha_{2}$ \\
\hline$G_{2}$ & $\alpha_{2}$ & -3 & $3 \alpha_{1}+2 \alpha_{2}$ \\
\hline
\end{tabular}




\section{Exceptional Lie Algebras}

\begin{tabular}{|c|c|c|c|c|}
\hline Type & $B_{1}$ & Orbit $(1)=G X$ & Orbit $(2)=G x$ & degree \\
\hline$E_{6}$ & & $\left(\begin{array}{c}20202 \\
2\end{array}\right)$ & $\left(\begin{array}{c}20202 \\
2\end{array}\right)$ & 2 \\
\hline$E_{6}$ & $-\left(\begin{array}{c}01111 \\
1\end{array}\right),-\left(\begin{array}{c}01210 \\
1\end{array}\right),-\left(\begin{array}{c}11110 \\
1\end{array}\right)$ & $\left(\begin{array}{c}21012 \\
1\end{array}\right)$ & $\left(\begin{array}{c}20202 \\
0\end{array}\right)$ & 3 \\
\hline$E_{6}$ & $-\left(\begin{array}{c}11100 \\
1\end{array}\right),-\left(\begin{array}{c}01111 \\
0\end{array}\right),-\left(\begin{array}{c}11110 \\
0\end{array}\right),-\left(\begin{array}{c}01110 \\
1\end{array}\right),-\left(\begin{array}{c}01210 \\
1\end{array}\right)$ & $\left(\begin{array}{c}11011 \\
1\end{array}\right)$ & $\left(\begin{array}{c}11011 \\
1\end{array}\right)$ & 5 \\
\hline$E_{6}$ & $-\left(\begin{array}{c}00110 \\
1\end{array}\right),-\left(\begin{array}{c}12321 \\
1\end{array}\right),-\left(\begin{array}{c}01100 \\
1\end{array}\right)$ & $\left(\begin{array}{c}10101 \\
0\end{array}\right)$ & $\left(\begin{array}{c}00200 \\
0\end{array}\right)$ & 7 \\
\hline$E_{7}$ & $-\left(\begin{array}{c}112111 \\
1\end{array}\right),-\left(\begin{array}{c}112210 \\
1\end{array}\right),-\left(\begin{array}{c}122110 \\
1\end{array}\right)$ & $\left(\begin{array}{c}210122 \\
1\end{array}\right)$ & $\left(\begin{array}{c}202022 \\
0\end{array}\right)$ & 3 \\
\hline$E_{7}$ & & $\left(\begin{array}{c}002020 \\
0\end{array}\right)$ & $\left(\begin{array}{c}002020 \\
0\end{array}\right)$ & 6 \\
\hline$E_{7}$ & $-\left(\begin{array}{c}111100 \\
1\end{array}\right),-\left(\begin{array}{c}134321 \\
2\end{array}\right),-\left(\begin{array}{c}111110 \\
0\end{array}\right)$ & $\left(\begin{array}{c}101012 \\
0\end{array}\right)$ & $\left(\begin{array}{c}002002 \\
0\end{array}\right)$ & 7 \\
\hline$E_{7}$ & & $\left(\begin{array}{c}000200 \\
0\end{array}\right)$ & $\left(\begin{array}{c}000200 \\
0\end{array}\right)$ & 13 \\
\hline$E_{7}$ & & $\left(\begin{array}{c}002000 \\
0\end{array}\right)$ & $\left(\begin{array}{c}002000 \\
0\end{array}\right)$ & 10 \\
\hline$E_{7}$ & $-\left(\begin{array}{c}011111 \\
1\end{array}\right),-\left(\begin{array}{c}111110 \\
1\end{array}\right),-\left(\begin{array}{c}111111 \\
0\end{array}\right),-\left(\begin{array}{c}012110 \\
1\end{array}\right)$ & $\left(\begin{array}{c}222020 \\
1\end{array}\right)$ & $\left(\begin{array}{c}222020 \\
1\end{array}\right)$ & 6 \\
\hline$E_{8}$ & $-\left(\begin{array}{c}222210 \\
1\end{array}\right),-\left(\begin{array}{c}1222111 \\
1\end{array}\right),-\left(\begin{array}{c}1232110 \\
1\end{array}\right),-\left(\begin{array}{c}1122211 \\
1\end{array}\right),-\left(\begin{array}{c}1232100 \\
2\end{array}\right)$ & $\left(\begin{array}{c}2101101 \\
1\end{array}\right)$ & $\left(\begin{array}{c}2020020 \\
0\end{array}\right)$ & 6 \\
\hline$E_{8}$ & $-\left(\begin{array}{c}1122100 \\
1\end{array}\right),-\left(\begin{array}{c}2464321 \\
3\end{array}\right),-\left(\begin{array}{c}0122110 \\
1\end{array}\right),-\left(\begin{array}{c}1111111 \\
1\end{array}\right)$ & $\left(\begin{array}{c}1010101 \\
0\end{array}\right)$ & $\left(\begin{array}{c}0020002 \\
0\end{array}\right)$ & 10 \\
\hline$E_{8}$ & $-\left(\begin{array}{c}1111100 \\
1\end{array}\right),-\left(\begin{array}{c}1343321 \\
2\end{array}\right),-\left(\begin{array}{c}2343221 \\
2\end{array}\right)$ & $\left(\begin{array}{c}1010100 \\
0\end{array}\right)$ & $\left(\begin{array}{c}1010100 \\
0\end{array}\right)$ & 14 \\
\hline$E_{8}$ & $-\left(\begin{array}{c}2354321 \\
2\end{array}\right),-\left(\begin{array}{c}1354321 \\
3\end{array}\right)$ & $\left(\begin{array}{c}0100100 \\
0\end{array}\right)$ & $\left(\begin{array}{c}0100100 \\
0\end{array}\right)$ & 22 \\
\hline$E_{8}$ & $-\left(\begin{array}{c}2465421 \\
3\end{array}\right),-\left(\begin{array}{c}1233221 \\
2\end{array}\right)$ & $\left(\begin{array}{c}0010010 \\
0\end{array}\right)$ & $\left(\begin{array}{c}0002000 \\
0\end{array}\right)$ & 16 \\
\hline$E_{8}$ & & $\left(\begin{array}{c}0020002 \\
0\end{array}\right)$ & $\left(\begin{array}{c}0020002 \\
0\end{array}\right)$ & 10 \\
\hline$E_{8}$ & $-\left(\begin{array}{c}1121111 \\
1\end{array}\right),-\left(\begin{array}{c}2465431 \\
3\end{array}\right),-\left(\begin{array}{c}0122111 \\
1\end{array}\right)$ & $\left(\begin{array}{c}101012 \\
0\end{array}\right)$ & $\left(\begin{array}{c}0020022 \\
0\end{array}\right)$ & 7 \\
\hline$E_{8}$ & $-\left(\begin{array}{c}1233211 \\
1\end{array}\right),-\left(\begin{array}{c}1232221 \\
1\end{array}\right),-\left(\begin{array}{c}1232211 \\
2\end{array}\right)$ & $\left(\begin{array}{c}2101222 \\
1\end{array}\right)$ & $\left(\begin{array}{c}2020222 \\
0\end{array}\right)$ & 3 \\
\hline$F_{4}$ & $-(1120),-(1221)$ & $(1012)$ & $(1012)$ & 3 \\
\hline$F_{4}$ & $-(1342),-(1110)$ & $(1010)$ & $(0200)$ & 4 \\
\hline$F_{4}$ & $-(0011)$ & $(0010)$ & $(0200)$ & 4 \\
\hline$F_{4}$ & & $(2200)$ & $(2200)$ & 3 \\
\hline$G_{2}$ & $-\left(3 \alpha_{1}+\alpha_{2}\right)$ & $(01)$ & $(02)$ & 1 \\
\hline$G_{2}$ & $-\left(\alpha_{1}+\alpha_{2}\right)$ & (10) & (02) & 1 \\
\hline
\end{tabular}




\section{Exceptional Lie Algebras}

\begin{tabular}{|c|c|}
\hline Type & Invariant parabolic $\tilde{B}^{\prime \prime}$ \\
\hline$E_{6}$ & $B_{0}$ \\
\hline$E_{6}$ & $\left\{-\alpha: \alpha \in B_{1}\right\}$ \\
\hline$E_{6}$ & $\left(\begin{array}{c}11100 \\
1\end{array}\right),\left(\begin{array}{c}11110 \\
0\end{array}\right),\left(\begin{array}{c}01111 \\
1\end{array}\right),\left(\begin{array}{c}01210 \\
0\end{array}\right)$ \\
\hline$E_{6}$ & $\left(\begin{array}{c}00110 \\
1\end{array}\right),\left(\begin{array}{c}01111 \\
0\end{array}\right),\left(\begin{array}{c}11111 \\
1\end{array}\right),\left(\begin{array}{c}11110 \\
1\end{array}\right),\left(\begin{array}{c}01100 \\
1\end{array}\right)$ \\
\hline$E_{7}$ & $\left\{-\alpha: \alpha \in B_{1}\right\}$ \\
\hline$E_{7}$ & $B_{0}$ \\
\hline$E_{7}$ & $\left(\begin{array}{c}111000 \\
1\end{array}\right),\left(\begin{array}{c}012210 \\
1\end{array}\right),\left(\begin{array}{c}112211 \\
1\end{array}\right),\left(\begin{array}{c}012111 \\
1\end{array}\right),\left(\begin{array}{c}111110 \\
0\end{array}\right)$ \\
\hline$E_{7}$ & $B_{0}$ \\
\hline$E_{7}$ & $B_{0}$ \\
\hline$E_{7}$ & $\left(\begin{array}{c}012111 \\
1\end{array}\right),\left(\begin{array}{c}111110 \\
1\end{array}\right),\left(\begin{array}{c}111111 \\
0\end{array}\right),\left(\begin{array}{c}012210 \\
1\end{array}\right)$ \\
\hline$E_{8}$ & $\left\{-\alpha: \alpha \in B_{1}\right\}$ \\
\hline$E_{8}$ & $\left(\begin{array}{c}1122100 \\
1\end{array}\right),\left(\begin{array}{c}0121111 \\
1\end{array}\right),\left(\begin{array}{c}1121110 \\
1\end{array}\right),\left(\begin{array}{c}1221100 \\
1\end{array}\right),\left(\begin{array}{c}0122110 \\
1\end{array}\right),\left(\begin{array}{c}1111111 \\
1\end{array}\right)$ \\
\hline$E_{8}$ & $\left(\begin{array}{c}1121100 \\
1\end{array}\right),\left(\begin{array}{c}0111110 \\
1\end{array}\right),\left(\begin{array}{c}1222211 \\
1\end{array}\right),\left(\begin{array}{c}1111000 \\
1\end{array}\right),\left(\begin{array}{c}0121111 \\
1\end{array}\right),\left(\begin{array}{c}1111110 \\
0\end{array}\right),\left(\begin{array}{c}0122100 \\
1\end{array}\right)$ \\
\hline$E_{8}$ & $\left(\begin{array}{c}1121110 \\
1\end{array}\right),\left(\begin{array}{c}1111100 \\
0\end{array}\right),\left(\begin{array}{c}0110000 \\
1\end{array}\right),\left(\begin{array}{c}0011111 \\
0\end{array}\right),\left(\begin{array}{c}1111000 \\
1\end{array}\right),\left(\begin{array}{c}0111110 \\
0\end{array}\right),\left(\begin{array}{c}0011100 \\
1\end{array}\right)$ \\
\hline$E_{8}$ & $\left(\begin{array}{c}0111100 \\
1\end{array}\right),\left(\begin{array}{c}0011111 \\
0\end{array}\right),\left(\begin{array}{c}1111000 \\
1\end{array}\right),\left(\begin{array}{c}0111110 \\
0\end{array}\right),\left(\begin{array}{c}0121000 \\
1\end{array}\right),\left(\begin{array}{c}1111100 \\
0\end{array}\right),\left(\begin{array}{c}0011110 \\
1\end{array}\right)$ \\
\hline$E_{8}$ & $B_{0}$ \\
\hline$E_{8}$ & $\left(\begin{array}{c}1221111 \\
1\end{array}\right),\left(\begin{array}{c}1122111 \\
1\end{array}\right),\left(\begin{array}{c}1233211 \\
2\end{array}\right),\left(\begin{array}{c}1232111 \\
1\end{array}\right),\left(\begin{array}{c}0122211 \\
1\end{array}\right)$ \\
\hline$E_{8}$ & $\left\{-\alpha: \alpha \in B_{1}\right\}$ \\
\hline$F_{4}$ & $(1220),(1122)$ \\
\hline$F_{4}$ & $(1120),(1111),(0121)$ \\
\hline$F_{4}$ & $(1220),(1110),(0011)$ \\
\hline$F_{4}$ & $B_{0}$ \\
\hline$G_{2}$ & $\alpha_{1}+\alpha_{2}$ \\
\hline$G_{2}$ & $\alpha_{1}+\alpha_{2}$ \\
\hline
\end{tabular}


Exceptional Lie Algebras

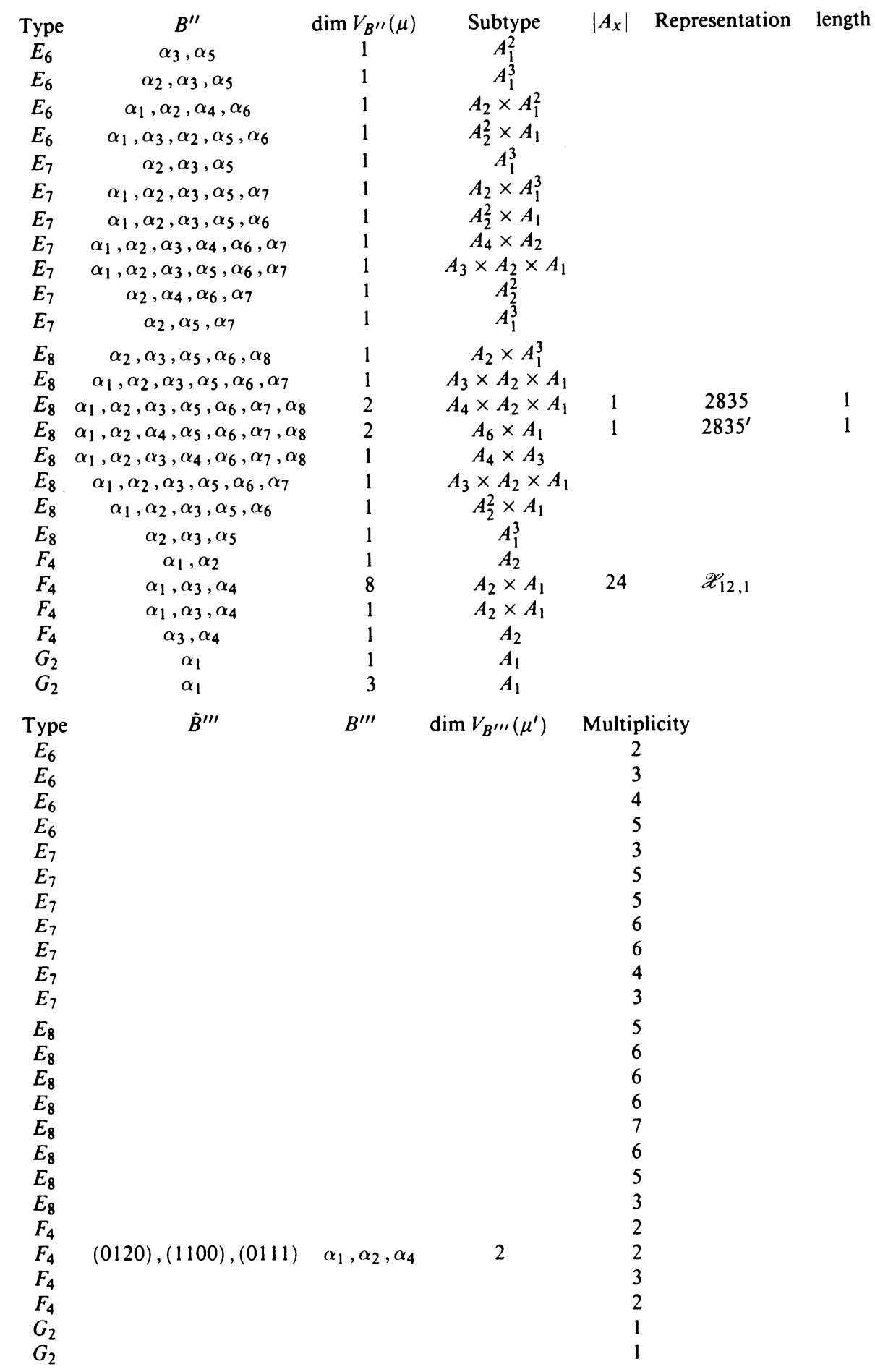




\section{REFERENCES}

1. D. Barbasch and D. Vogan, Primitive ideals and orbital integrals in complex classical groups, Math. Ann. 259 (1982), 153-199.

2. $\ldots$, Primitive ideals and orbital integrals in complex exceptional groups, J. Algebra 80 (1983), 350-382.

3. W. M. Beynon and G. Lusztig, Some numerical results on characters of exceptional Weyl groups, Math. Proc. Cambridge Philos. Soc. 84 (1978), 417-426.

4. W. Borho and J.-L. Brylinski, Differential operators on homogeneous spaces. I, Invent. Math. 69 (1982), 437-476.

5. W. Borho and J.-C. Jantzen, Über primitive Ideale in der Einhüllenden einer halbeinfachen Lie-Algebra, Invent. Math. 39 (1977), 1-53.

6. W. Borho and R. MacPherson, Partial resolutions of nilpotent varieties, Astérisque 101 (1983), 23-74.

7. N. Bourbaki, Groupes et algèbres de Lie, Chaps. IV-VI, Hermann, Paris, 1968.

8. R. W. Carter, Finite groups of Lie type, Wiley, New York, 1985.

9. N. Conze-Berline and M. Duflo, Sur les représentations induites des groupes semi-simples complexes, Compositio Math. 34 (1977), 307-336.

10. J. Dixmier, Enveloping algebras, North-Holland, Amsterdam, 1977.

11. O. Gabber and A. Joseph, On the Bernstein-Gelfand-Gelfand resolution and the Duflo sum formula, Compositio Math. 43 (1981), 107-131.

12. R. K. Gupta, Copies of the adjoint representation inside induced ideals, preprint, Paris, 1985.

13. R. Hotta, On Joseph's construction of Weyl group representations, Tôhoku Math. J. 36 (1984), 49-74.

14. J.-C. Jantzen, Kontravariante Formen auf induzierten Darstellungen halbeinfacher Lie-Algebren, Math. Ann. 226 (1977), 53-65.

15. __ Moduln mit einem hochsten Gewicht, Lecture Notes in Math., vol. 750, Springer-Verlag, Berlin and New York, 1979.

16. __ Einhullende Algebren halbeinfacher Lie-Algebren, Springer-Verlag, Berlin, 1983.

17. A. Joseph, The minimal orbit in a simple Lie algebra and its associated maximal ideal, Ann. Sci. Ecole Norm. Sup. 9 (1976), 1-30.

18. _ Goldie rank in the enveloping algebra of a semi-simple Lie algebra. I, II, III, J. Algebra 65 (1980), 269-283; 284-306; 73 (1981), 295-326.

19. Kostant's problem, Goldie rank and the Gelfand-Kirillov conjecture, Invent. Math. 56 (1980), 191-213.

20. __ On the variety of a highest weight module, J. Algebra 88 (1984), 238-278.

21. _ On the associated variety of a primitive ideal, J. Algebra 93 (1985), 509-523.

22. $\ldots$, On the cyclicity of vectors associated with Duflo involutions, Non-Commutative Harmonic Analysis, Lecture Notes in Math., vol. 1243, Springer-Verlag, Berlin, 1987, pp. 145-188.

23. __ A criterion for an ideal to be induced, J. Algebra 110 (1987), 480-497.

24. __ Dixmier's problem for Verma and principal series submodules, J. London Math. Soc. 20 (1979), 193-204.

25. G. Lusztig, Irreducible representations of finite classical groups, Invent. Math. 43 (1977), 125175.

26. _ A class of irreducible representations of a Weyl group. I, II, Proc. Kon. Nederl. Akad. A82 (1979), 323-335; A85 (1982), 219-226.

27. __ Characters of reductive groups over a finite field, Ann. of Math. Studies, no. 107, Princeton Univ. Press, Princeton, N.J., 1984.

28. __ Sur les cellules gauches des groupes de Weyl, C. R. Acad. Sci. Paris 302 (1986), 5-8.

29. G. Lusztig and N. Spaltenstein, Induced unipotent classes, J. London Math. Soc. (2) 19 (1979), 41-52. 
30. I. G. Macdonald, Some irreducible representations of the Weyl groups, Bull. London Math. Soc 4 (1972), 148-150.

31. N. Spaltenstein, On the fuxed point set of a unipotent element on the variety of Borel subgroups, Topology 16 (1977), 203-204.

32. T. Tanisaki, Private communication.

33. A. Joseph, Completion functors in the $\mathfrak{O}$ category, Lecture Notes in Math., vol. 1020, SpringerVerlag, Berlin and New York, 1983, pp. 80-106.

Department of Theoretical Mathematics, The Weizmann Institute of Science, ReHOVOT 76100 , ISRAEL

Laboratoiré de Mathematiques Fondamentales (Equipe de Recherche Associée au C.N.R.S.), Université de Pierre et Marie Curie, France 